\title{
Anti-Apoptosis and Regeneration in the Visual System: Effects of BAG1 (Bcl-2-associated athanogene-1)
}

\author{
Dissertation \\ zur Erlangung des Doktorgrades \\ der Mathematisch-Naturwissenschaftlichen Fakultäten \\ der Georg-August-Universität zu Göttingen
}

vorgelegt von

Anne-Véronique Planchamp

aus Vevey (Schweiz)

Göttingen 2007 


\section{D7}

Referent:

Korreferent:

Tag der mündlichen Prüfung:
Prof. Hardeland

Prof. Braus

01.11.2007 
A mes parents

\author{
A la mémoire de mon \\ Grand-papa Emmanuel 1
}




\section{Declaration}

I hereby declare that the thesis "Anti-Apoptosis and Regeneration in the Visual System: Effects of BAG1 (Bcl-2-associated athanogene-1)" has been written independently and with no other sources and aids than quoted.

Anne-Véronique Planchamp

Göttingen, 2007 


\section{Table of contents}

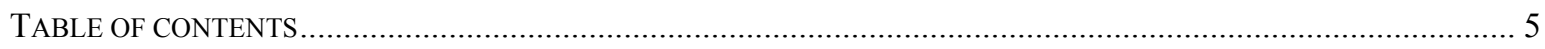

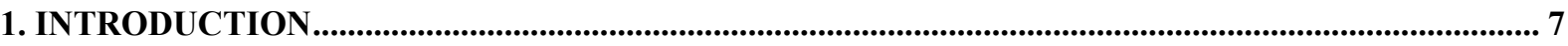

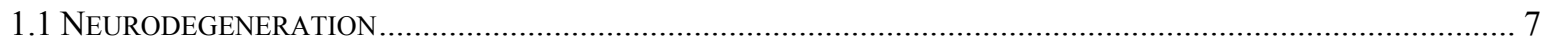

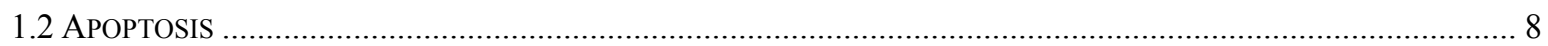

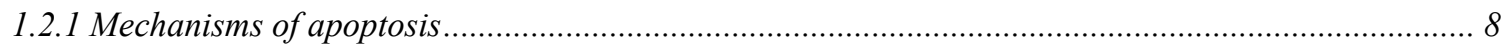

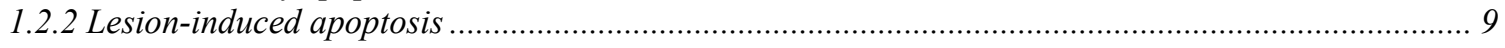

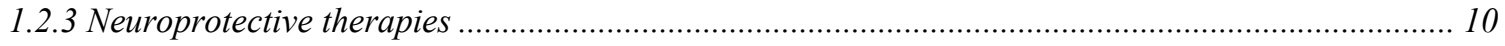

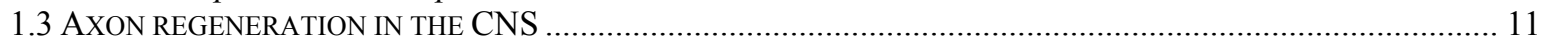

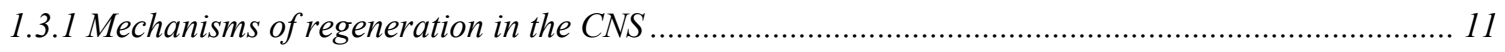

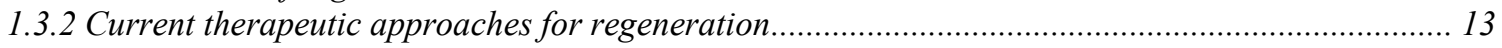

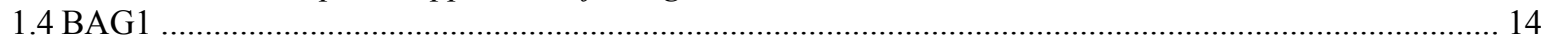

1.5 RAF-1 KINASE

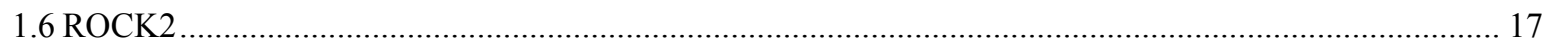

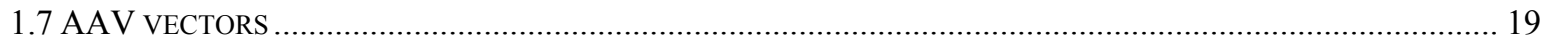

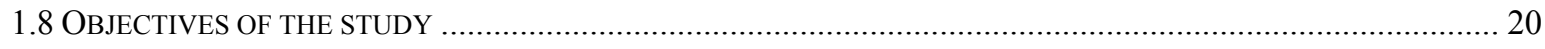

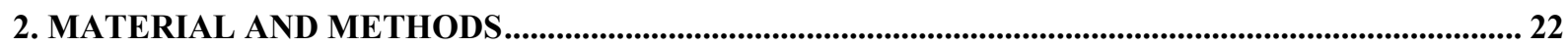

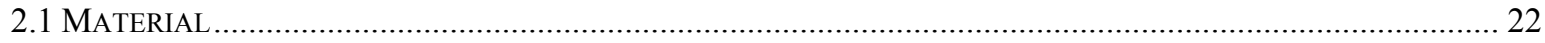

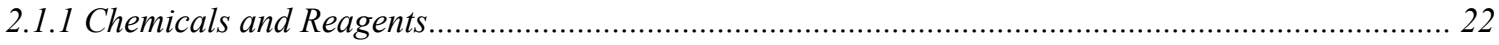

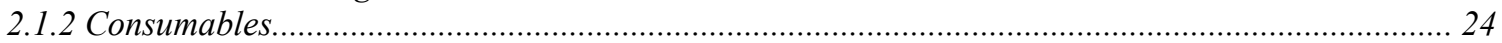

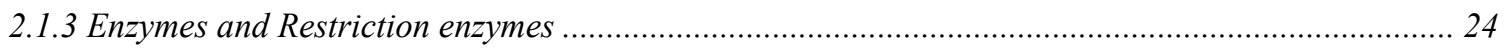

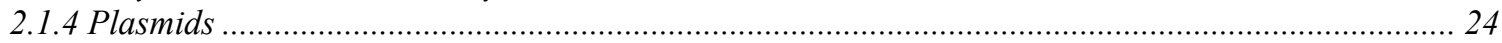

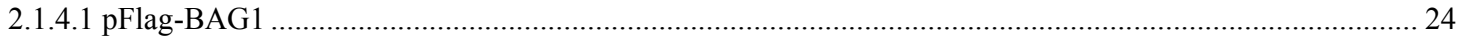

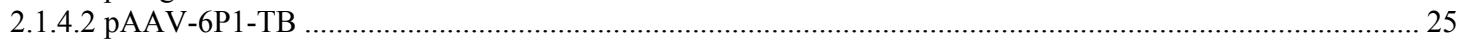

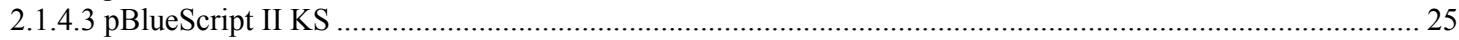

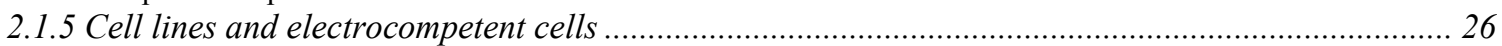

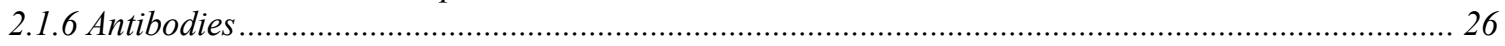

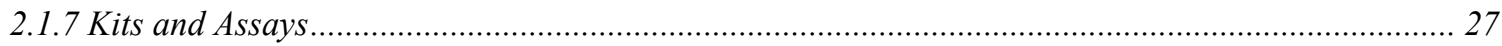

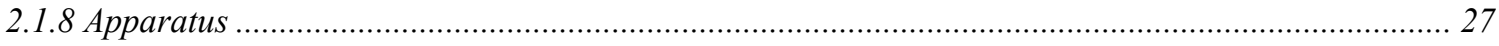

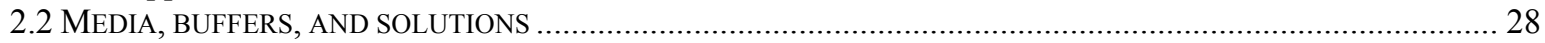

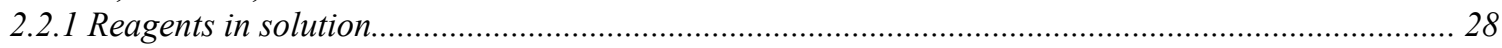

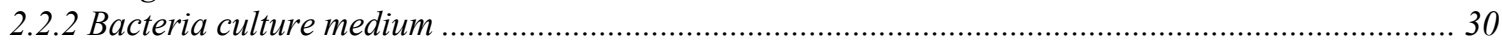

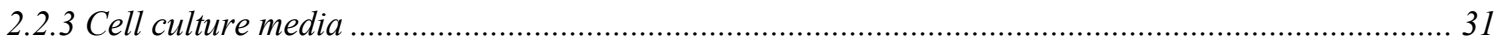

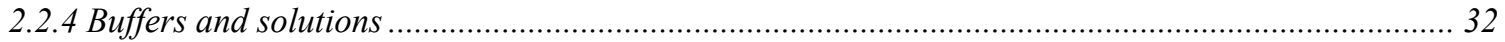

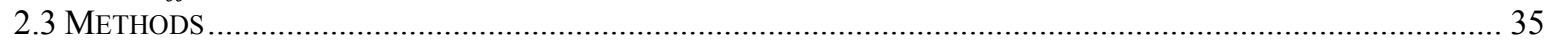

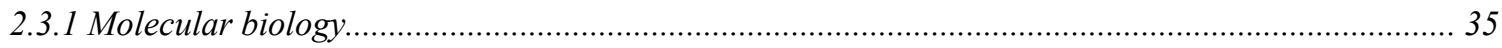

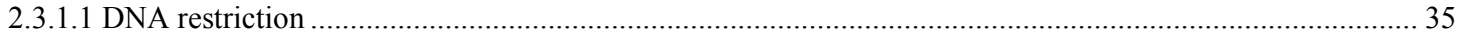

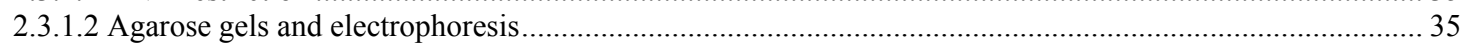

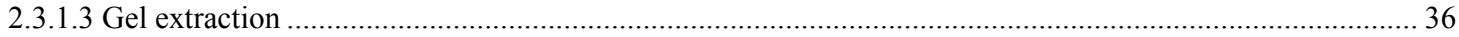

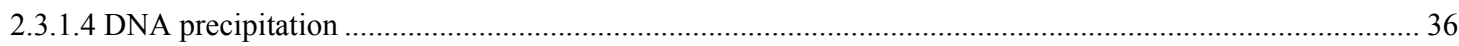

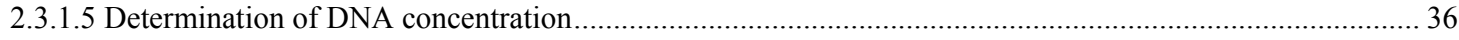

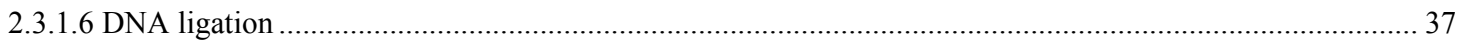

2.3.1.7 Production of chemically competent cells and bacterial transformation............................................... 37

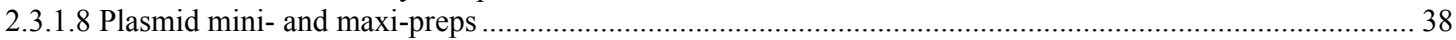

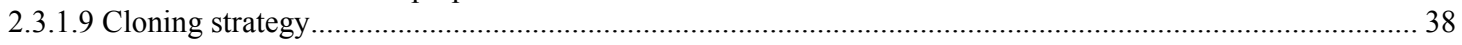

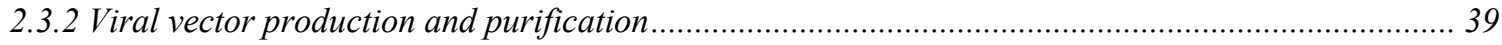

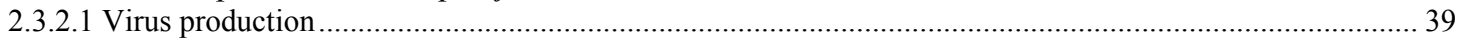

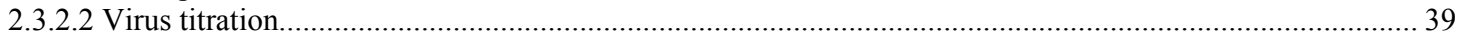

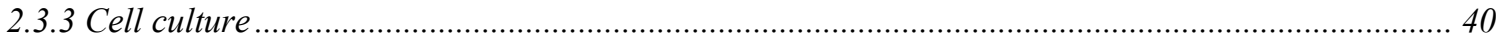

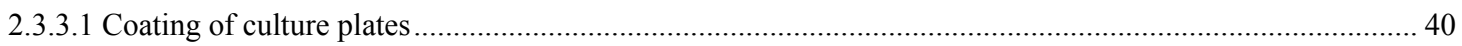

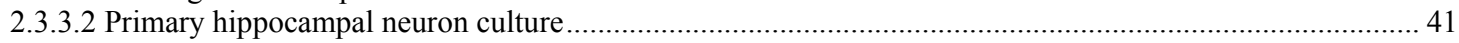

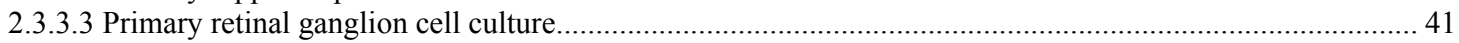

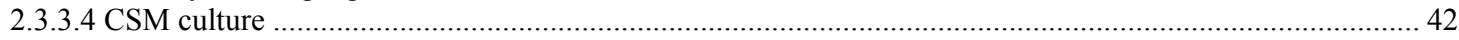

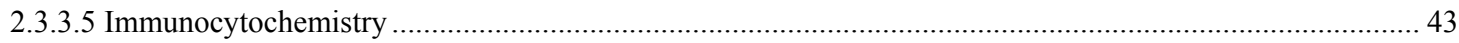

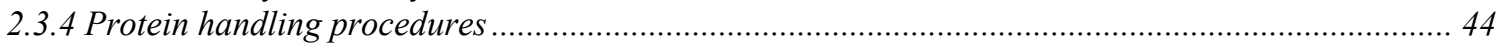

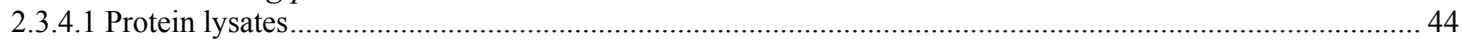

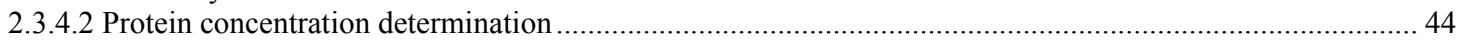

2.3.4.3 Sodium dodecyl sulphate-polyacrylamide gel electrophoresis (SDS-PAGE) .......................................... 44

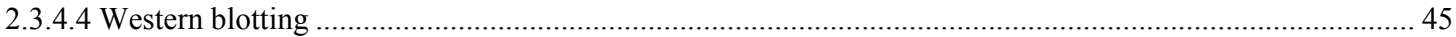

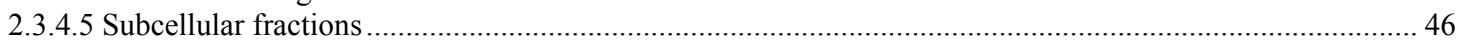




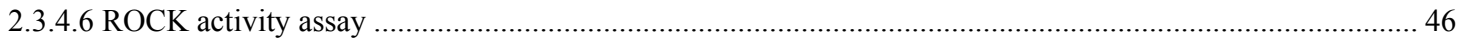

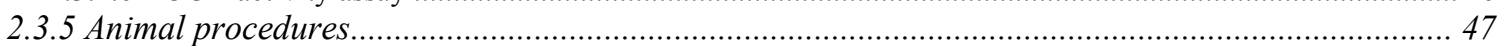

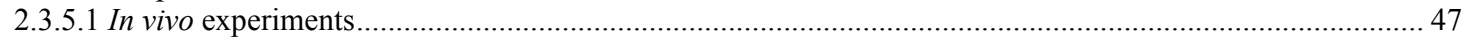

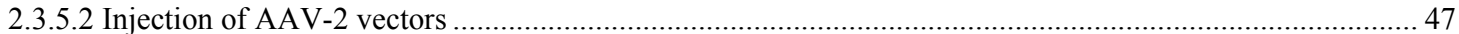

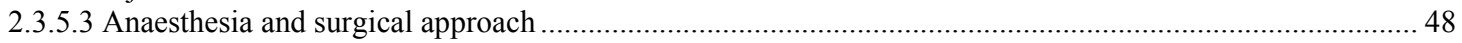

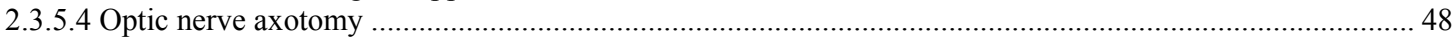

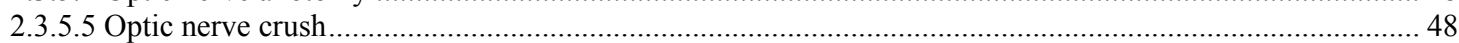

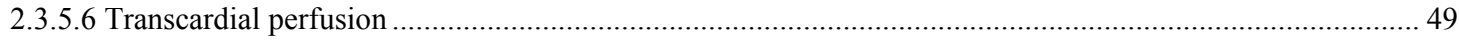

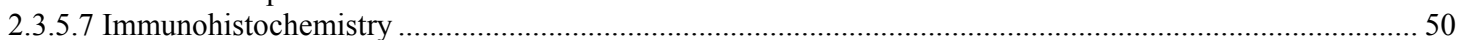

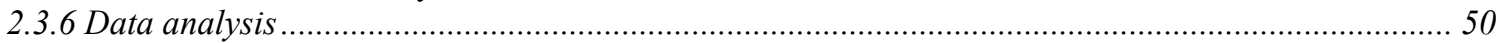

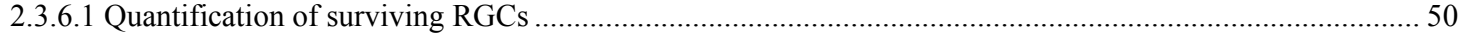

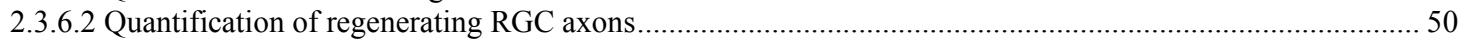

2.3.6.3 Quantification of neurite length in primary RGCs........................................................................ 51

2.3.6.4 Quantification growth cone surface and lamellipodia in primary RGCs .................................................... 51

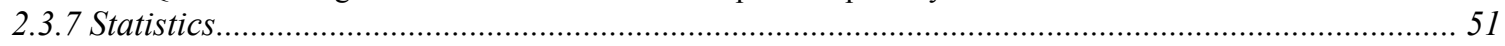

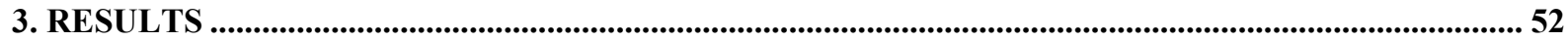

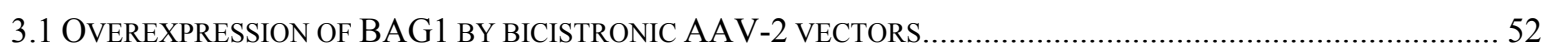

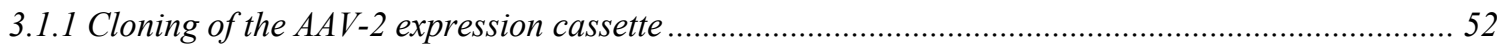

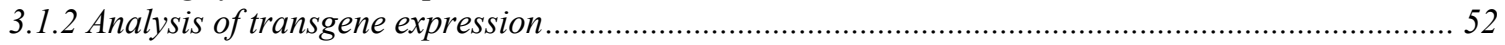

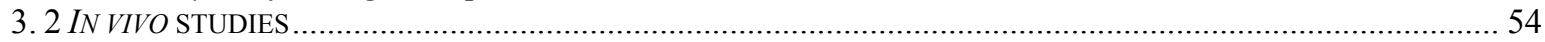

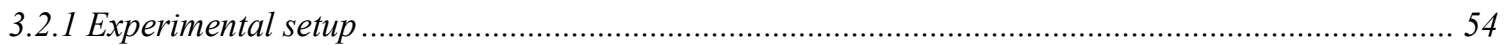

3.2.2 RGC survival after axotomy-induced apoptosis is increased by BAG1 ...................................... 54

3.2.3 BAG1 increases number and length of regenerating axons after optic nerve crush ....................... 56

3.3 IN VITRO STUDIES: EFFECTS OF BAG1 OVEREXPRESSION ON PRIMARY RGCS ...................................... 58

3.3.1 Neurite length and growth cone surface of primary $R G C s$ are increased by $B A G 1$...................... 58

3.3.2 Translocation of Raf-1 and ROCK2 in primary RGCs after BAG1 overexpression......................... 61

3.4 IN VITRO STUDIES: EFFECTS OF BAG1 OVEREXPRESSION IN CSM CELLS.................................................62

3.4.1 BAG1 induces Raf-1 and ROCK2 translocation in CSM cells: western blot analysis...................... 62

3.4.2 BAG1 induces Raf-1 and ROCK2 translocation in CSM cells: immuno-cytochemical analysis ........ 64

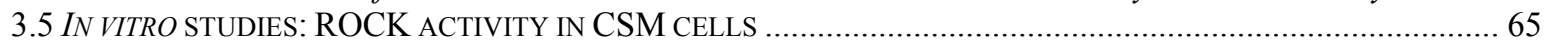

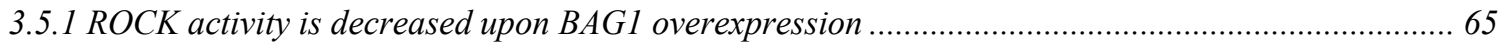

3.5.2 BAG1-mediated decrease in ROCK activity is Raf-1-dependent .............................................. 66

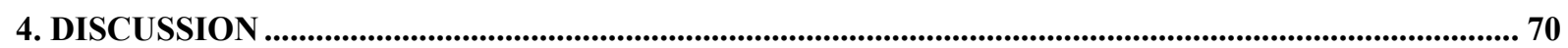

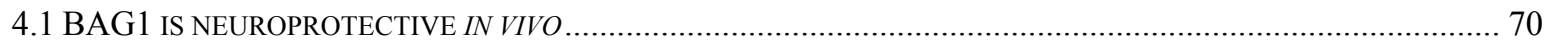

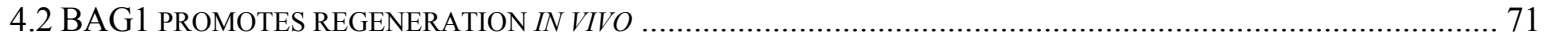

4.3 SUBCELLULAR TRANSLOCATION OF RAF-1 AND ROCK2 BY BAG1 .................................................. 71

4.4 REGULATION OF ROCK ACTIVITY BY BAG1 VIA RAF-1 .................................................................. 72

4.4.1 Involvement of Raf-1 and the mitogen-activated protein kinase (MAPK) pathway....................... 72

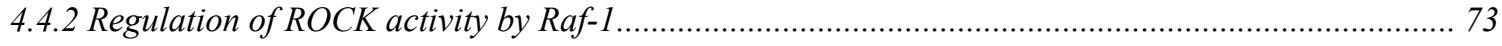

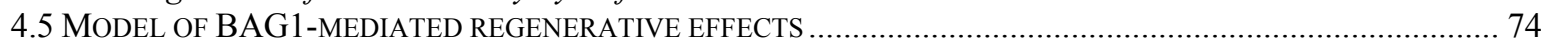

4.6 NEUROPROTECTION AND REGENERATION: ACHIEVEMENTS AND PERSPECTIVES ....................................... 76

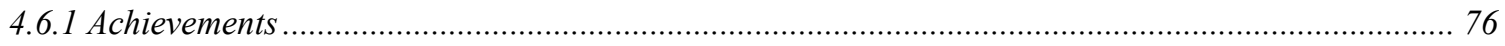

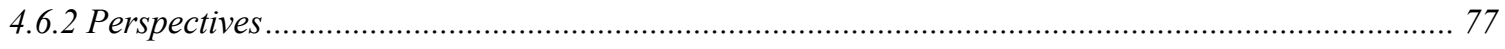

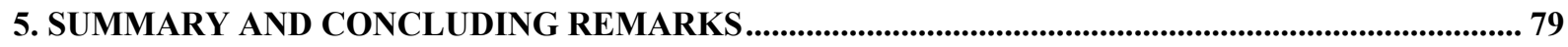

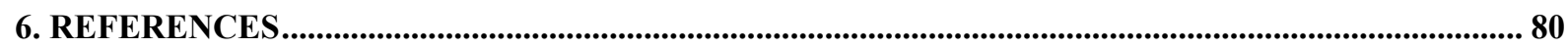

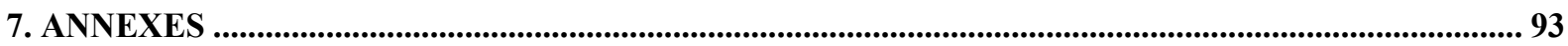

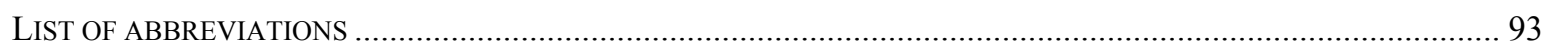

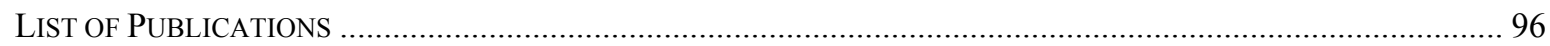

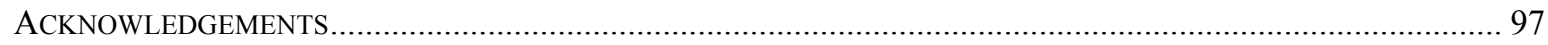

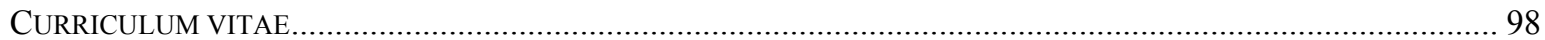




\section{Introduction}

\subsection{Neurodegeneration}

In Western countries, neurodegenerative diseases like Parkinson's (PD) or Alzheimer's (AD) diseases are a major cause of morbidity. The prevalence of Parkinson's disease in Europe is about 1.8 percent for people over 65 years (de Rijk et al., 2000), while 5 percent of people aged 65 to 74 suffer from Alzheimer's disease. Beyond the age of 85, AD reaches nearly half of the population (from NIA's Progress Report on Alzheimer's disease, 1998). Due to increased life expectancy, there is a continuous rise in incidence and prevalence of neurodegenerative diseases. The cellular and molecular mechanisms of all these neurodegenerative diseases are still poorly understood. However, a better understanding of the neurobiological principles underlying these diseases would contribute to the development of efficient therapies targeting the causes of diseases themselves. To date, only few symptomatic therapies, such as Levodopa and other dopaminergic drugs for the treatment of disorders of movement associated with PD, are available. The constant increase in the affected population, together with the lack of curative treatment, contributes to high costs for health care budgets in developed countries. Scientific and socio-economic interests therefore meet for developing future therapeutic strategies.

Axonal degeneration is a characteristic feature of neurodegenerative diseases, as well as traumatic nerve injury. It often precedes cell loss and has been proposed as an early indicator of subsequent degenerative disease progression (Coleman and Perry, 2002). Current experimental models and therapies mostly target two separate aspects, either the prevention of cell death or the promotion of regeneration. Inhibition of cell-death alone, however, is insufficient for the restoration of functional neurons. On the other hand, regeneration strongly depends on the presence of living cell bodies. Regeneration failure can be attributed to lesioninduced apoptosis, to a diminished intrinsic growth capacity of adult neurons or to growth inhibition by a non-permissive adult central nervous system (CNS) environment. In order to restore neuronal functionality, research and neuroregenerative therapies should therefore aim at combining both aspects. In a model of traumatic injury of the optic nerve, we studied the effects of a protein known to be involved in both antiapoptotic and regenerative processes. 


\subsection{Apoptosis}

\subsubsection{Mechanisms of apoptosis}

Apoptosis, first described by Kerr and colleagues in 1972 (Kerr et al., 1972) is a wellconserved genetic pathway common to all metazoans and is necessary for normal tissue development and homeostasis (Danial and Korsmeyer, 2004). It is induced by activation of cysteine proteases which cleave target proteins at specific aspartate residues (caspases). Apoptosis can be triggered by two distinct and evolutionary conserved pathways. The extrinsic pathway involves cell-surface death receptors such as Tumour Necrosis Factor $\alpha(\mathrm{TNF} \alpha)$ or Fas, while the intrinsic pathway implicates mitochondria and endoplasmatic reticulum (Danial and Korsmeyer, 2004; Foo et al., 2005). Both pathways converge in the activation of effector caspases-3, 6 and 7 (Figure 1).

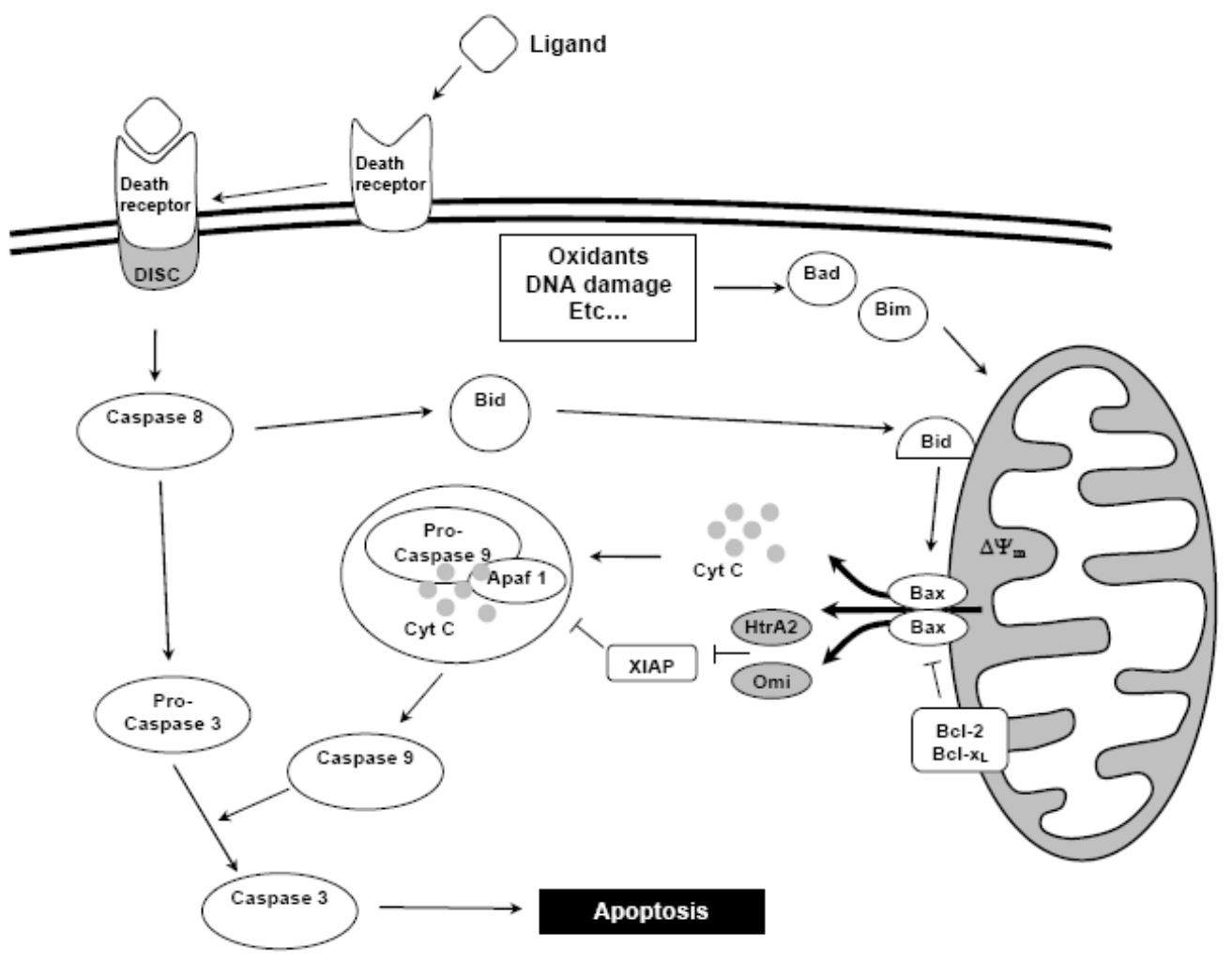

Figure 1: $\quad$ Scheme of major apoptosis pathways. Upon ligand binding to the death receptor, caspase-8 can directly activate caspase-3 (extrinsic pathway) or indirectly activate the intrinsic pathway via Bid translocation to the mitochondrion. Subsequent Bak/Bax oligomerisation results in release of pro-apoptotic molecules and activation of the apoptosome. See text (section 1.2) for details. Pointed arrow, activation; bar end, inhibition; round-shaped, pro-apoptotic protein; square-shaped, anti-apoptotic protein; $\Delta \Psi_{\mathrm{m}}$, mitochondrial membrane potential. (Modified from Bähr and Lingor, 2006). 
Upon receptor binding, adaptor proteins recruit caspase-8 to the death-inducing signalling complex (DISC), allowing its dimerisation and activation. Activated caspase- 8 can directly activate caspase-3, which cleaves various cellular cytoplasmic proteins and fragments nuclear DNA. Additionally, caspase- 8 acts indirectly on the mitochondria via activation of Bid and thereby links extrinsic and intrinsic pathways.

The intrinsic pathway plays an important role in transmitting and amplifying death signals. Stress stimuli including oxidants or DNA damage can activate pro-apoptotic BH3-only proteins of the Bcl-2 family such as Bid, Bad, and Bim. Upon activation, Bid translocates to the mitochondria and activates Bax and Bak (Li et al., 1998; Luo et al., 1998; Gross et al., 1999), leading to pore formation and release of pro-apoptotic molecules such as cytochrome c, Smac/DIABLO and Omi/HtrA2 (Antonsson et al., 2000). Bax/Bak activity can be antagonised by anti-apoptotic Bcl-2 family proteins such as Bcl-2 and Bcl- $\mathrm{X}_{\mathrm{L}}$ (Cheng et al., 2001). Mitochondrial permeabilisation can also occur in response to intrinsic permeability regulation, changes in inner mitochondrial membrane following increased cytosolic $\mathrm{Ca}^{2+}$ levels or oxidative stress. As a result, these changes lead to a loss of mitochondrial membrane potential, mitochondrial swelling and release of cytochrome c (Antonsson et al., 2000).

Release of cytochrome c induces formation of the apoptosome, a complex constituted of Apaf-1, cytochrome c, dATP and caspase-9 (Zou et al., 1999). The apoptosome promotes caspase- 9 activation by proteolytic cleavage of its precursor procaspase-9, and amplifies death signalling by direct activation of caspase-3 (Hakem et al., 1998). The final steps of apoptosis take place when critical levels of activated caspase-3 are reached, and can be antagonised by $\mathrm{X}$-chromosome linked inhibitor of apoptosis protein (XIAP). The latter may itself be inhibited by Smac and Omi (Du et al., 2000; Verhagen et al., 2000; Martins et al., 2002). This final process is triggered by caspases-8, 9, and 10 and is characterised by shrinkage of the cell, membrane inversion and exposure of phosphatidylserine (Kerr et al., 1972; Vaux and Korsmeyer, 1999). Further characteristics are cell blebbing, nuclear fragmentation, chromatin condensation and DNA degradation. Finally, the apoptotic bodies are engulfed by phagocytes (Krammer, 1999), thus preventing inflammation in the surrounding tissues.

\subsubsection{Lesion-induced apoptosis}

Axotomy of the optic nerve is a widely used model for the study of apoptosis in the CNS (Villegas-Perez et al., 1993; Berkelaar et al., 1994; Garcia-Valenzuela et al., 1994; Isenmann 
and Bähr, 1997; Isenmann et al., 1997). The selective neuronal death occurring within two weeks after transection of the optic nerve affects $90 \%$ of retinal ganglion cells (RGCs) and exhibits features similar to apoptosis. It is characterised by cell shrinkage, DNA fragmentation and degradation of organelles, while no inflammation in surrounding tissues is observed. Lesion-induced apoptosis is signalled through the same pathways as developmentally programmed cell death (Potts et al., 1982). Optic nerve transection of adult RGCs induces loss of Bcl-2 and Bcl- $\mathrm{X}_{\mathrm{L}}$, and increased Bax expression (Antonsson et al., 2001). Furthermore, axotomy activates caspase-3 and 9 (Kermer et al., 1999; Kermer et al., 2000), which is accompanied by an increase in c-Jun expression (Hull and Bähr, 1994; Robinson, 1994).

Finally, $\mathrm{Ca}^{2+}$ influx is also observed following trauma, as well as in ischemia and degenerative diseases (Choi, 1988). In explanted retinas of new-born rat pups, a rise in intracellular $\mathrm{Ca}^{2+}$ correlates with cell death (Cellerino et al., 2000a). $\mathrm{Ca}^{2+}$ is thought to play a role in apoptosis by modifying phosphorylation states of proteins and modulating signalling pathways (Ermak and Davies, 2002).

\subsubsection{Neuroprotective therapies}

The enzymatic activity of activated caspases can be inhibited by synthetic peptide caspase inhibitors such as DEVD-fmk, zLEHD-fmk and zIETD-fmk (specific for caspase-3, 9 and 8 respectively). In traumatic nerve injury models, intraocular injection of such inhibitors prevents RGC cell death, especially at early time points (Chaudhary et al., 1999). However, this approach offers only a temporary delay before neurons undergo cell-death through alternative pathways (Kermer et al., 1999). Other physiological inhibitors such as members of the IAP family, e.g. XIAP, are also able to prevent neuronal apoptosis (Kügler et al., 2001). Moreover, down-regulation of c-Jun, Apaf-1 and Bax using RNA interference was shown to protect RGCs from axotomy-induced cell-death (Lingor et al., 2005). One major drawback of such strategies is that they target only downstream levels of the apoptosis cascade by inhibiting the final execution steps of the pathway. While these approaches keep cell bodies alive, it does not allow for a regrowth of neuronal processes to restore functional connectivity. Caspase inhibition thus does not seem the appropriate candidate for therapeutic approaches.

Survival of CNS neurons depends on various different growth factors (Oppenheim, 1996) and may be stimulated by neurotrophic supply. Intraocular administration of brain-derived 
neurotrophic factor (BDNF) was shown to rescue RGCs in vivo (Mey and Thanos, 1993), while adenovirus-mediated administration of glial cell line-derived neurotrophic factor (GDNF), ciliary neurotrophic factor (CNTF) and XIAP enhances survival of axotomised RGCs (Peinado-Ramon et al., 1996; Klocker et al., 1997; Di Polo et al., 1998; Koeberle and Ball, 1998; Weise et al., 2000; Schmeer et al., 2002).

\subsection{Axon regeneration in the CNS}

\subsubsection{Mechanisms of regeneration in the CNS}

Adult neurons of the CNS show only a limited regenerative ability after mechanic or metabolic injury, which is insufficient for restoration of functionality. This can be attributed to lesion-induced neuronal cell death or a diminished intrinsic growth capacity of adult neurons, but also to growth inhibition by a non-permissive environment.

In contrast to embryonic and peripheral nervous system (PNS) neurons, adult neurons of the CNS retain only a limited capacity for regrowth, but can extend neurites over long distances in the permissive environment of a peripheral nerve graft (David and Aguayo, 1981). However, in an injured environment, they fail to regenerate beyond the lesion site (Tom et al., 2004). These differential regenerative responses are mainly a consequence of the environmental properties (Schwab and Thoenen, 1985). Axon regeneration might be restricted by persistence or re-expression of many inhibitory or repulsive guidance cues involved in axon path finding during development, such as members of the Ephrin (e.g. Ephrin A4, B3, Goldshmit et al., 2004; Benson et al., 2005) and Semaphorin (e.g. Sema5A in RGCs, Goldshmit et al., 2004) families. Upon injury, the glial environment of adult CNS, especially the myelin structure formed by oligodendrocytes, can be damaged. This leads to the exposure of myelin-derived outgrowth inhibitors such as Nogo, myelin-associated glycoprotein (MAG), and oligodendrocyte myelin glycoprotein (OMgp, Filbin, 2003; Yiu and He, 2003). Moreover, the glial scar itself can act as a barrier to axon regrowth by recruiting microglia, oligodendrocyte precursors and astrocytes to the lesion site (Silver and Miller, 2004). These isolate and minimise the area of inflammation and cellular degeneration, but also secrete inhibitory extracellular matrix (ECM) molecules such as chondroitin sulphate proteoglycans (CSPGs, Niederost et al., 1999), among them aggrecan and brevican, which strongly impair axon regrowth (McKeon et al., 1991; Fawcett, 1997; Fitch and Silver, 1997; Huber and Schwab, 2000). 
In the CNS, Nogo-A, MAG and OMgp inhibit neurite outgrowth and induce growth cone collapse (GrandPre et al., 2000; GrandPre et al., 2002) by interaction with the $\mathrm{NgR} / \mathrm{p} 75$ complex (Fournier et al., 2001; Barton et al., 2003; Filbin, 2003; He et al., 2003; Yiu and He, 2003). Upon binding to the NgR GPI-protein receptors and its co-receptors (e.g. TNFR, p75, TROY, LINGO-1), myelin-based inhibitory molecules signal through pathways involving $\mathrm{Ca}^{2+}$ or activation of RhoA and ROCK (Figure 2, Bandtlow et al., 1993; Niederost et al., 2002; Wong et al., 2002; Filbin, 2003; Fournier et al., 2003; Park et al., 2005; Shao et al., 2005; Yiu and He, 2006). This leads to the phosphorylation and subsequent inactivation of cofilin by LIM kinase (Yang et al., 1998; Hsieh et al., 2006). Cofilin is a regulator of actin filament dynamics, whose inactivation results in actin polymerisation and stabilisation of the growth cone cytoskeleton of damaged axons ( $\mathrm{Ng}$ and Luo, 2004). Their regenerative outgrowth is consequently restricted (Jalink et al., 1994; Lehmann et al., 1999). Additionally, the Rho/ROCK pathway also mediates inhibition of neurite outgrowth exerted by CPSGs (Monnier et al., 2003).

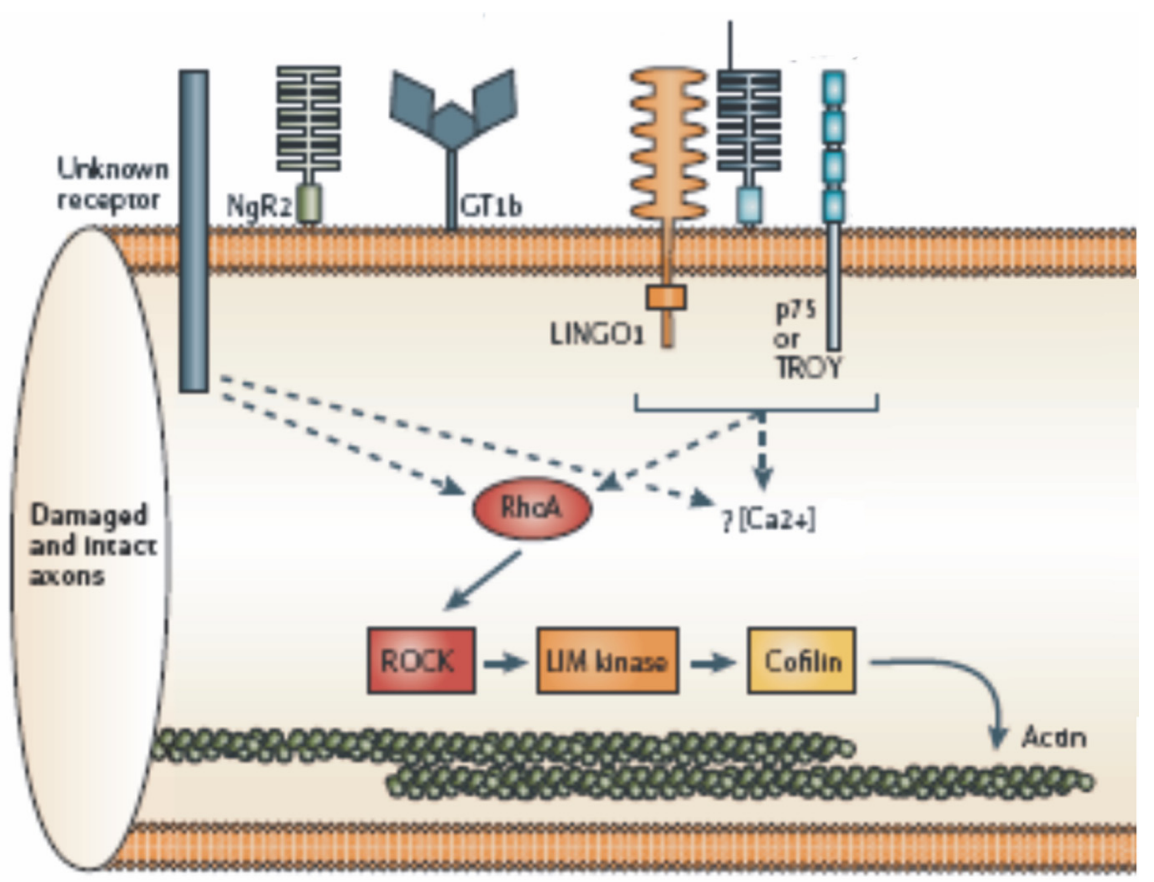

Figure 2: Inhibitory signalling pathways involved in axon growth and regeneration. Upon binding to the NgR GPI-protein receptors and its co-receptors, myelin-based inhibitory signals activate RhoA and ROCK2, which in turn phosphorylates LIM kinase. Phosphorylation of cofilin by LIM kinase inhibits its actin depolymerising activity, resulting in stabilisation of growth cone cytoskeleton and limited regenerative outgrowth. (Modified from Yiu and He, 2006). 


\subsubsection{Current therapeutic approaches for regeneration}

In comparison to the PNS, expression or up-regulation of regeneration-associated genes responsible for the intrinsic regenerative capacity of PNS upon injury is weaker or more transient in CNS neurons. For example, growth-associated protein 43 (GAP43) is upregulated in PNS upon injury and increases the cell intrinsic capability to regenerate (Skene and Virag, 1989), while in axotomised CNS neurons, its increase is transient (Fernandes et al., 1999). GAP43 overexpression alone, however, is not sufficient for regeneration in the CNS (Mason et al., 2000; Bomze et al., 2001; Leaver et al., 2006b). The transcription factor c-Jun was shown to be required for survival and regeneration of rat pheochromocytoma-12 cells, rat dorsal root ganglion neurons and c-Jun ${ }^{-/}$transgenic mice (Dragunow et al., 2000; Raivich et al., 2004; Lindwall and Kanje, 2005b, 2005a)

Neurotrophic factors can improve regrowth to a certain extent, probably by increasing the neuron intrinsic growth capacity via activation of transcription cascades resulting in the simultaneous expression of growth associated genes such as GAP43 or c-Jun (Van Hooff et al., 1989; Schreyer and Skene, 1993; Broude et al., 1997; Roux et al., 2004). For example, neuronal growth factor (NGF) enhances sensory axon elongation and arborisation (Diamond et al., 1992; Tucker et al., 2001), as well as outgrowth of dorsal root ganglion neurons in culture (Paves and Saarma, 1997). BDNF and GDNF were shown to promote elongation of RGCs in vivo (Cohen-Cory and Fraser, 1995) and neuronal regrowth in the spinal cord (Iannotti et al., 2003), respectively.

Inhibition of myelin-derived outgrowth inhibitors is not sufficient to enhance regeneration. MAG knock-out mice and mutant mice lacking one or all 3 Nogo isoforms (Nogo-A, B, C) show only moderate or no increase in regeneration after spinal cord injury (Bartsch et al., 1995; Kim et al., 2003; Zheng et al., 2003). NgR-deficient mice exhibit persistent axonal growth inhibition on myelin in vitro, while axons of p75NTR deficient mice, though less inhibited by myelin in vitro, show no improvement in regeneration of corticospinal tract axons after spinal hemisection (Zheng et al., 2005).

A further promising target is the inhibition of inhibitory signalling pathways such as the Rho/ROCK cascade. Application of Rho-inactivating enzyme C3 transferase overcomes growth inhibition on inhibitory substrates and promotes axon regeneration past the lesion site (Lehmann et al., 1999; Dergham et al., 2002; Niederost et al., 2002; Fournier et al., 2003; Bertrand et al., 2005). Inhibition of ROCK by pharmacological inhibitors increases the 
number and length of regenerating RGCs after optic nerve crush in vivo (Lingor et al., 2007) and counteracts inhibitory signalling by CSPGs in vitro (Monnier et al., 2003; Lingor et al., 2007).

\subsection{BAG1}

Bcl-2-associated athanogene-1 (BAG1) is a member of the Hsp70/Hsc70-binding protein family (Takayama et al., 1995; Zeiner and Gehring, 1995) and has first been described as Bcl2 interacting protein. BAG1 has been mostly studied because of its function as a co-chaperone of the Hsp70/Hsc70 family. However, it is also known to interact with a large number of other cellular targets (Townsend et al., 2003), including elements of the proteasome system, such as Ubiquitin and the co-chaperone/E3 ligase C-terminus of Hsp70-interacting protein (CHIP). Furthermore, Raf-1 (Takayama et al., 1995; Bardelli et al., 1996; Kullmann et al., 1998; Liu et al., 1998; Matsuzawa et al., 1998), steroid hormone E receptors and nuclear hormone receptors (Knee et al., 2001) have been reported to interact with BAG1.

Overexpression of BAG1 results in enhanced tumour cell proliferation, cell motility and increased resistance to apoptosis (Takayama et al., 1995; Bardelli et al., 1996; Clevenger et al., 1997; Takayama et al., 1999). Neurite length and generation time were significantly increased upon BAG1 transfection in vitro (Kermer et al., 2002). Neuroprotective effects were observed in BAG1 overexpressing mice, which showed a reduction in the ischemic lesion volume following middle cerebral artery occlusion (Kermer et al., 2003). BAG1 ${ }^{-/-}$mice die during embryogenesis due to failed neurogenesis, suggesting an important role for BAG1 in neuronal differentiation and neuronal survival (Watanabe et al., 2001; Götz et al., 2005). BAG1 also coordinates the cellular signals that determine the growth state and differentiation of the cell according to its environmental and physiological state (Song et al., 2001), for instance by interacting with and activating the Ser/Thr-specific protein kinase Raf-1 (Zeiner and Gehring, 1995; Wang et al., 1996; Song et al., 2001; Kermer et al., 2002). BAG1 interacts with and regulates its different molecular targets most probably through promotion of conformational changes (Takayama and Reed, 2001). Cell type and intracellular location determine the specific effects of BAG1 (Kermer et al., 2002).

Like the other members of the BAG family, BAG1 comprises an ubiquitin-like domain (ULD) and a BAG domain, which is a conserved region of approximately 70 amino residues located at the C-terminus (Figure 3, Takayama et al., 1995; Takayama and Reed, 2001). 


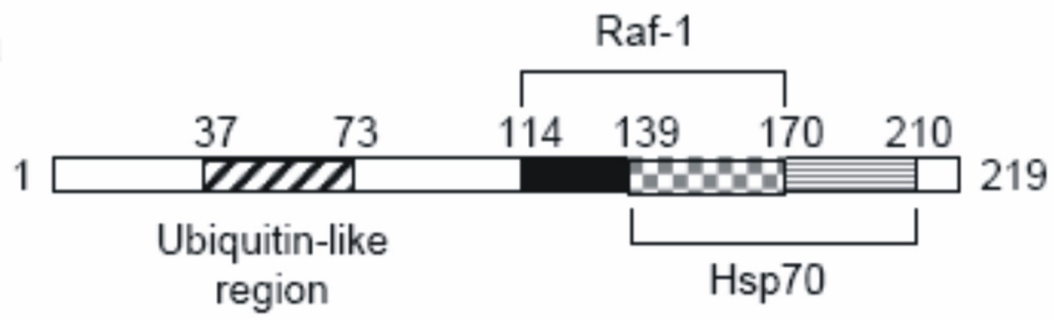

Figure 3: $\quad$ Structure of the mouse BAG1 short isoform. Striped box, ubiquitin-like region, black box, Raf-1 binding site; grey box, Hsp70-binding site. Numbers indicate amino residues. (From Song et al., 2001).

The ULD domain is required for protein targeting to the proteasome and strongly contributes to the anti-apoptotic function of BAG1 (Bardelli et al., 1996; Takayama et al., 1997; Luders et al., 1998). The BAG domain contains several binding sites, allowing interaction with proteins such as Bcl-2, Hsp70 and Raf-1. Binding to the ATPase domain of Hsp70 via residues 139 to 210 stimulates ADP release and enhances the chaperone ATPase activity (Hohfeld and Jentsch, 1997; Sondermann et al., 2001; Alberti et al., 2002). The Hsp70 binding sequence has been shown to overlap with another binding site (residues 114 to 170) allowing recruitment and activation of Raf-1 kinase, a protein important for cell proliferation and differentiation (Wang et al., 1996). This allows BAG1 to act as a molecular switch. Under physiological conditions, it constitutively binds to Raf-1 and promotes cell proliferation. In contrast, under stress conditions BAG1 acts as a co-chaperone.

\subsection{Raf-1 kinase}

Raf-1 (or c-Raf, Jansen et al., 1983; Rapp et al., 1988) is the best studied member of a small family of highly conserved Ser/Thr protein kinases comprising two further isoforms, A-Raf and B-Raf. All isoforms differ in their expression profile, regulation and function but share a common structure constituted of three conserved regions (CR, Crocoll et al., 2000, Figure 4). The CR1 contains a RasGTP-loaded-binding site domain (RBD) and a cysteine-rich domain (CRD), while CR2 is a domain rich in Ser/Thr residues. CR3 comprises the catalytic kinase domain. The C-terminal region of Raf-1 additionally contains several phosphorylation and binding sites responsible for the fine regulation of its catalytic activity, which depends on various interacting partners and the signalling pathways involved (Janssens and Goris, 2001; Ceulemans and Bollen, 2004; reviewed in Dhillon et al., 2007). 


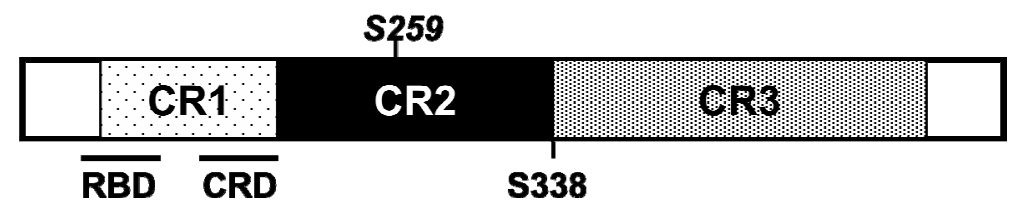

Figure 4: $\quad$ Schematic depiction of the structure of Raf-1. Residues involved in the activation of Raf-1 are indicated in bold and dotted boxes, while inhibitory residues are indicated in cursive. RBD, Rho-binding domain; CRD, cysteine-rich domain. (Modified from Baccarini, 2005).

Usually, Raf-1 exists in an inactive conformation which is promoted by its N-terminal autoinhibitory domain masking the kinase domain (Chong and Guan, 2003). This conformation is stabilised by binding of phosphokinase 14-3-3 (Dhillon and Kolch, 2002; Chong and Guan, 2003). Raf-1 activity is stimulated by many signals including growth factors, tumour promoters, inflammatory cytokines, calcium mobilisation, DNA damaging agents and oxygen radicals (Prouty et al., 1998). Raf-1 activation occurs in two steps. Firstly, autoinhibition from the N-terminal region on the C-terminal region is neutralised by dephosphorylation of Ser259. In a second step, Raf-1 activity is increased by multiple phosphorylations in the C-terminal catalytic region, in particular at Ser338. Among others, the phosphorylated active Ser338 residue is responsible for the repression of the autoinhibition exerted by the N-terminal regulatory region (Tran and Frost, 2003). Furthermore, it regulates the binding of the downstream effector mitogen-activated and extracellular regulated kinase (MEK, Xiang et al., 2002; Park et al., 2006), and the translocation of Raf-1 to mitochondria which is important for its anti-apoptotic function (Alavi et al., 2003; Jin et al., 2005). Activated Raf-1 binds to and phosphorylates MEK, which in turn phosphorylates and activates the extracellular signal-regulated kinases (ERK) $1 / 2$, leading to the expression of transcription factors such as Ets, Elk or Myc, or targeting of other cellular substrates (Figure $5)$.

BAG1-mediated Raf-1 activation is Ras-independent and subsequently activates the downstream ERK pathway, resulting in cell growth and differentiation (Wang et al., 1996; Song et al., 2001). Additionally, it is possible that BAG1 modulates Raf-1 subcellular targeting and function through its interactions with transmembrane proteins such as Bcl-2 (Leevers et al., 1994; Stokoe et al., 1994). 


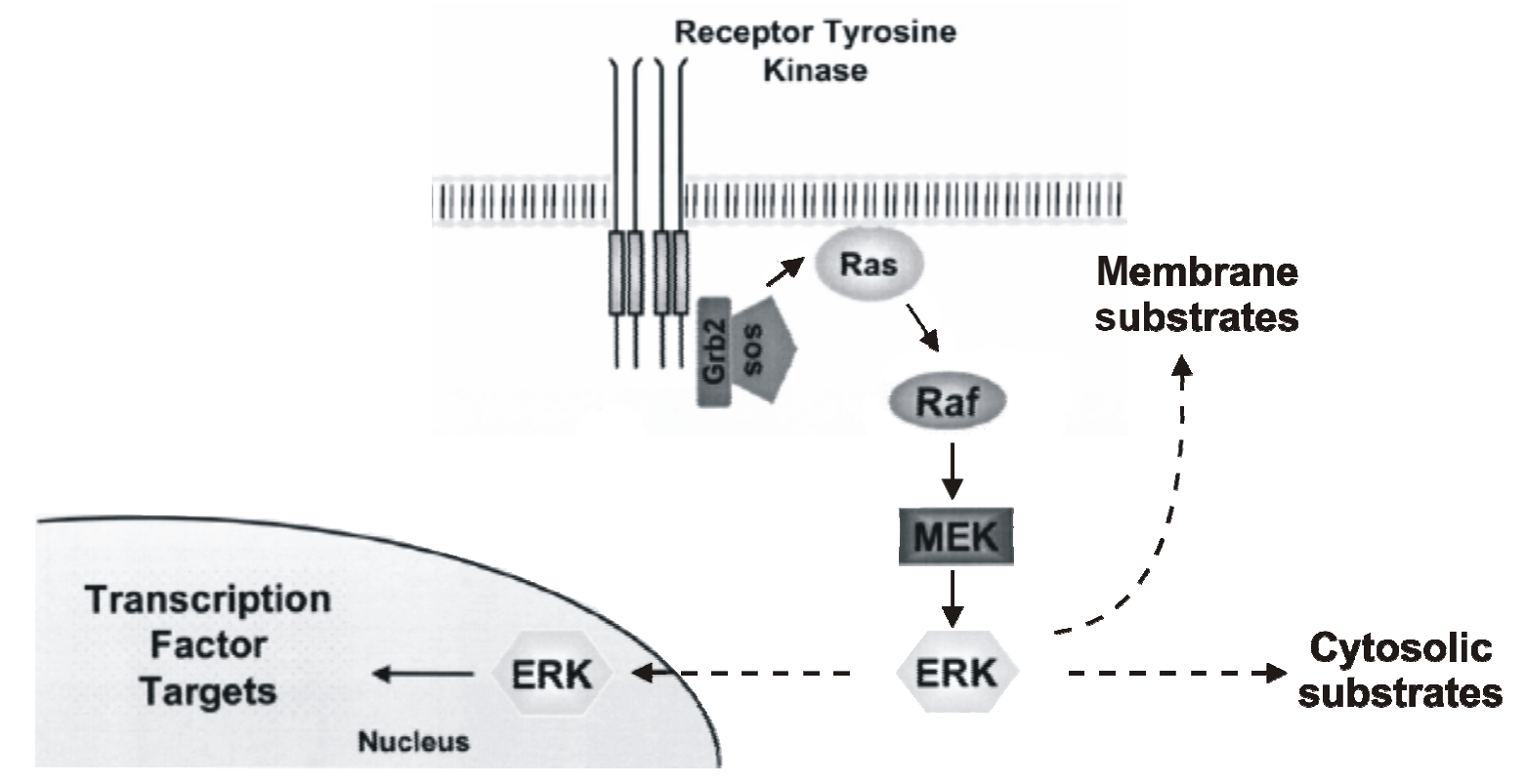

Figure 5: $\quad$ Schematic depiction of the Ras-Raf-ERK pathway. The ERK cascade is initiated by the GTPase Ras, which recruits Raf- 1 to the membrane and activates the Raf-1 kinase. Active Raf1 binds to and phosphorylates MEK, which in turn phosphorylates ERK1/2. This leads to the expression of transcription factors or targeting of different membrane and cytosolic substrates. (Modified from Juliano et al., 2004).

Activation of Raf-1 is specifically involved in axon elongation of sensory neurons, which is triggered by microtubule polymerisation and phosphorylated (active) ERK2 translocation to the nucleus (Markus et al., 2002). Raf-1 was also shown to be sufficient to induce neuronal differentiation of rat hippocampal cells (Kuo et al., 1996). Furthermore, Raf-1 plays an essential role in maintaining the organisation of the cytoskeleton, and thus in cell polarity and migration. Recently, Raf-1 was shown to interact with ROCK2, a major component of the growth inhibitory signalling pathway (Ehrenreiter et al., 2005), and to negatively regulate its activity (Baccarini, 2005). In these experiments, conditional gene ablation demonstrated that Raf-1 knock-out cells exhibit ROCK2 hyperactivity and mislocalisation at the membrane. These changes lead to a deregulation of both the actin and vimentin cytoskeleton. The latter is a main constituent of intermediate filaments.

\subsection{ROCK2}

ROCK2, a member of the Rho-associated kinases family (Ishizaki et al., 1996; Leung et al., 1996; Matsui et al., 1996; Nakagawa et al., 1996), is a coiled-coil protein kinase of $160 \mathrm{kDa}$ constituted of three main domains (Figure 6). The N-terminal catalytic domain of approximately 300 amino residues contains a Ser/Thr motif, while the central coiled-coil domain includes a Rho-binding domain (RBD) and mediates interactions with other $\alpha$-helical 
proteins. The RBD comprises about 80 amino residues and is only known to interact with activated RhoA and RhoC (Fujisawa et al., 1996). A C-terminal pleckstrin-homology (PH) domain, containing a cysteine-rich region, participates in interactions with different lipid mediators and might play a role in protein localisation (Chen et al., 2002; Kawabata et al., 2004; Yoneda et al., 2005). Additionally, the RBD and PH domains interact with the kinase domain by forming an autoinhibitory loop.

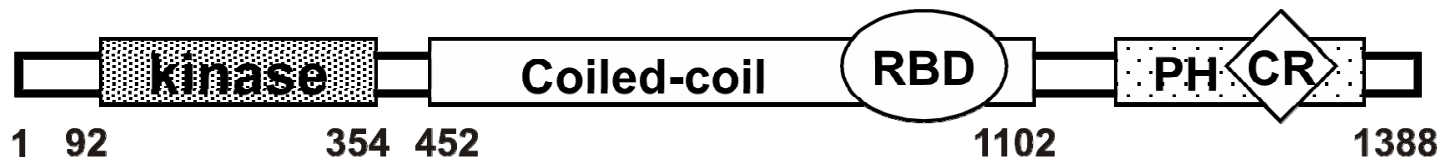

Figure 6: Schematic depiction of the structure of ROCK2. Numbers indicate amino residues. RBD, Rho-binding domain; PH, pleckstrin homology domain; CR, cysteine-rich domain. (From Shi and Wei, 2007).

ROCK 2 has been mostly described as the downstream effector of the Rho pathway and plays an essential role in the regulation of the actin cytoskeleton (Narumiya et al., 1997). Upon Rho activation, ROCK2 phosphorylates LIM kinase (Maekawa et al., 1999; Ohashi et al., 2000; Sumi et al., 2001), which in turn phosphorylates cofilin. Phosphorylation of cofilin leads to its subsequent inactivation and F-actin stabilisation (Figure 7, Müller et al., 2005).

Within the cascade triggered by myelin-derived inhibitors of axonal growth, ROCK2 has a pivotal regulatory role in transmission of the inhibitory signal from the small GTPase RhoA to LIM kinase, which in turn regulates actin homeostasis (Schwab, 2004).

ROCK2-mediated phosphorylation leads to inactivation of the myosin light chain (MLC), inhibition of MLC phosphatase, or activation of myosin ATPase (Amano et al., 1996; Kimura et al., 1996; Kureishi et al., 1997; Kawano et al., 1999). This stimulates actomyosin contraction (Ishizaki et al., 1996; Leung et al., 1996; Amano et al., 1997), thus modulating neurite retraction (Amano et al., 1996; Kureishi et al., 1997; Hirose et al., 1998) or membrane blebbing (Mills et al., 1998). ROCK2 can also mediate cytoskeleton reorganisation, growth cone collapse and microtubule dynamics through regulation of the collapsin response mediator protein-2 (Arimura et al., 2000), the microtubule-associated protein MAP2 and Tau (Amano et al., 2003), or the Ezrin-Radixin-Moesin family. The latter proteins are highly present at actin-rich structures and thought to link actin to the plasma membrane (Tsukita et al., 1997; Bretscher, 1999). 


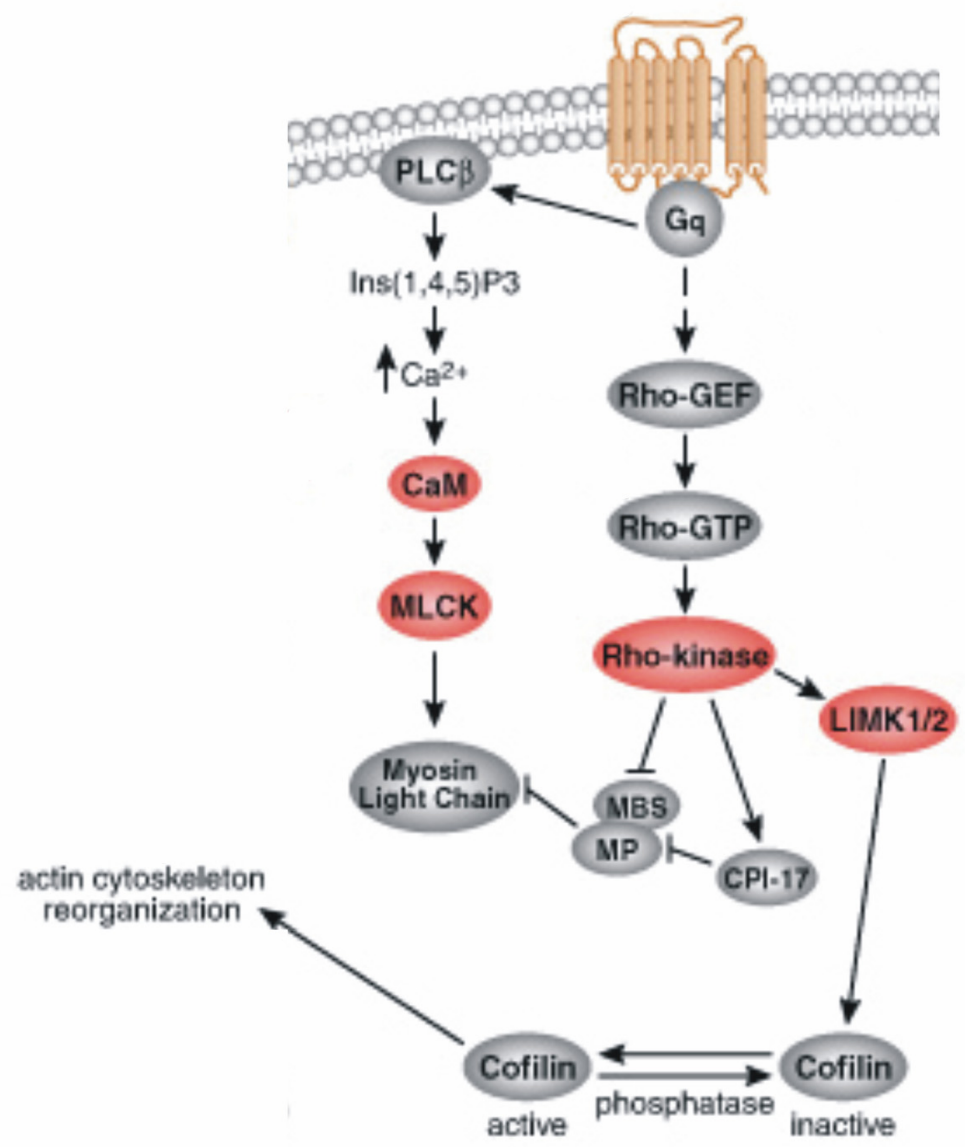

Figure 7: $\quad$ RhoA/ROCK2 signalling pathway. Gq, heterotrimeric G protein; PLC $\beta$, phospholipase C $\beta$; MBS, myosin-biding subunit of myosin phosphatase; MP, myosin phosphatase; CPI-17, protein kinase $\mathrm{C}$-potentiated inhibitory protein for heterotrimeric myosin phosphatase of 17 $\mathrm{kDa}$; Ins $(1,4,5) \mathrm{P}_{3}$, inositol triphosphate; CaM, calmoduline; MLCK, myosin light chain kinase. See text (section 1.6) for details. (Modified from Cell Signaling Technologies).

\subsection{AAV vectors}

Access to the CNS for therapeutic purposes is limited, mainly because of the presence of the blood brain barrier, which permits only the passage of molecules of certain size and polarity to the brain parenchyma. Moreover, systemic application of therapeutic molecules, whose half-life might be short and their expression subsequently transient, offers no specific targeting to subpopulations of cells, especially to neurons. In order to transfect the lesioned CNS, efficient gene transfer tools which allow for specific cell-targeting and long-lasting expression and delivery are necessary. These features are exhibited by viral vectors.

Adeno-associated viruses (AAV) are small dependoviruses from the Parvoviridae family, which can only replicate in the presence of adenovirus, herpes virus or vaccinia virus (Buller et al., 1981). The AAV genome $(4.675 \mathrm{~kb})$ contains inverted terminal repeats of 145 
nucleotides (Srivastava et al., 1983) and two large non-overlapping open reading frames encoding viral capside proteins (cap) and proteins necessary for gene replication and transcription (rep). Differences in the cap gene sequences account for the many AAV serotypes (AAV-1 to AAV-9, AAV-Rh10, Cearley and Wolfe, 2006) and their different transduction properties.

AAV's recombinant genome contains no viral genes except the inverted terminal repeat sequences (ITR) necessary for its replication. It especially lacks the rep gene responsible for site-specific integration in chromosomal DNA, and thus remains episomal. This makes AAV one of the safest viral vectors. Additionally, AAV shows no vector toxicity and no immunogenicity. Furthermore, wild-type AAV is not associated with any known disease in humans. Thus, AAV appears as a promising vector for therapeutic use in humans.

AAV possesses many advantages, including stable and long-lasting transgene expression (up to one year, Lackey et al., 2000; Stieger et al., 2006), and the ability to infect both dividing and non-dividing cells (Flotte et al., 1994; Flotte et al., 2004). However, its packaging capacity (less than $5 \mathrm{~kb}$ ) is relatively small. Cell type specificity and regulation of gene expression (kinetics, specificity of expression) can be achieved through the use of appropriate serotypes and cell specific promoters. For example, targeted expression in neurons can be achieved by means of the human synapsin promoter (Kügler et al., 2001; Kügler et al., 2003b).

\subsection{Objectives of the study}

To summarise, CNS neurons may undergo apoptosis or can survive after axonal injury, but they fail to regenerate beyond the lesion site, leaving intact cell bodies deprived of their functionality. To date, experimental and therapeutic approaches are based either on prevention of apoptosis or promotion of neuronal regeneration. Survival strategies result in stabilisation of cell bodies already in the process of cell-death, but are not alone sufficient to promote regeneration and functional recovery (Mansour-Robaey et al., 1994). On the other hand, regenerative strategies strongly require the presence of intact cell bodies in large numbers. Therefore, one promising approach is the combination of both aspects. Our aim in the present work was to enhance neuronal functionality after traumatic nerve injury by combining antiapoptotic and pro-regenerative strategies. In order to achieve this goal, we overexpressed a protein known to be involved in both processes. 
Here, we focus on AAV-mediated BAG1 overexpression in the RGC system and investigated its antiapoptotic and regenerative effects in two different in vivo paradigms, the optic nerve axotomy and crush models. To further elucidate the mechanisms of action underlying BAG1 regenerative effects, we went on to examine the effects of BAG1 in vitro in two different cell types. The primary rat retinal ganglion cells are the closest in vitro model of RGCs, exhibiting long axons allowing for protein localisation studies and in vitro assessment of regeneration and axonal morphology. The rat neuronal CSM cell line, stably transfected with BAG1, presents larger cell bodies and is easily maintained in culture. These features allowed us to examine more precisely the molecular mechanisms of BAG1-mediated regenerative response. 


\section{Material and Methods}

\subsection{Material}

\subsubsection{Chemicals and Reagents}

If not mentioned otherwise, chemicals were purchased from AppliChem. Reagents used in this study are listed in Table 1.

\begin{tabular}{|c|c|}
\hline Product & Reference \\
\hline 2-Propanol & AppliChem, Darmstadt, Germany \\
\hline 2 - $\beta$-mercaptoethanol & Sigma, Taufkirchen, Germany \\
\hline Acetone & AppliChem, Darmstadt, Germany \\
\hline Agar & Merck, Darmstadt, Germany \\
\hline Agarose & AppliChem, Darmstadt, Germany \\
\hline Ammonium peroxide & Roth, Karlsruhe, Germany \\
\hline Ammonium peroxidase & Merck, Darmstadt, Germany \\
\hline Ampicilline & Sigma, Taufkirchen, Germany \\
\hline B-27 supplement & Gibco, Karlsruhe, Germany \\
\hline Bicinchoninic acid (BCA) & Sigma, Taufkirchen, Germany \\
\hline Brain-derived neurotrophic factor (BDNF) & Tebu, Offenbach, Germany \\
\hline Borate & AppliChem, Darmstadt, Germany \\
\hline Bromophenol blue sodium salt & Serva, Heidelberg, Germany \\
\hline Bovine serum albumine (BSA) & Sigma, Taufkirchen, Germany \\
\hline Chloral hydrate & Fluka, Seelze, Germany \\
\hline Ciliary neurotrophic factor (CNTF) & Tebu, Offenbach, Germany \\
\hline Complete protease inhibitor & Roche, Mannheim, Germany \\
\hline Coumaric acid & Fluka, Taufkirchen, Germany \\
\hline Cryomatrix & Thermoshandon, Frankfurt/Main, Germany \\
\hline $\mathrm{D}(+)$-glucose & Sigma, Taufkirchen, Germany \\
\hline $\mathrm{D}(+)$-sucrose & AppliChem, Darmstadt, Germany \\
\hline Diethyl pyrocarbonate (DEPC) & Sigma, Taufkirchen, Germany \\
\hline Diethylether & Riedel-de-Haen, Seelze, Germany \\
\hline Dimethyl sulfoxide (DMSO) & AppliChem, Darmstadt, Germany \\
\hline $\begin{array}{l}\text { Dulbecco's modified Eagle's medium } \\
\text { (DMEM) }\end{array}$ & Gibco, Karlsruhe, Germany \\
\hline DMEM F:12 (1:1) & Gibco, Karlsruhe, Germany \\
\hline DNase & Roche, Mannheim, Germany \\
\hline $\begin{array}{l}\text { Dulbecco's Phosphate Buffered Saline } \\
\text { (DPBS) }\end{array}$ & Gibco, Karlsruhe, Germany \\
\hline Dual marker & BioRad, Munich, Germany \\
\hline Earle's Buffered Salt Solution (EBSS) & Gibco, Karlsruhe, Germany \\
\hline Ethylenediamine-tetraacetic acid (EDTA) & Sigma, Taufkirchen, Germany \\
\hline $\begin{array}{l}\text { Ethylenglycol-bis-(b-aminoethylether) N, } \\
\text { N, N', N'-tetraacetic acid (EGTA) }\end{array}$ & Sigma, Taufkirchen, Germany \\
\hline Ethanol absolute & AppliChem, Darmstadt, Germany \\
\hline Ethidium bromide & Sigma, Taufkirchen, Germany \\
\hline Foetal calf serum (FCS) & PAA, Coelbe, Germany \\
\hline Formaldehyde & Merck, Darmstadt, Germany \\
\hline
\end{tabular}




\begin{tabular}{|c|c|}
\hline Forskolin & Sigma, Taufkirchen, Germany \\
\hline Gene Ruler $^{\mathrm{TM}} 100$ bp DNA Ladder Plus & Fermentas, St. Leon-Rot, Germany \\
\hline Gene Ruler $^{\mathrm{TM}} 1 \mathrm{~kb}$ DNA Ladder & Fermentas, St. Leon-Rot, Germany \\
\hline Glycerine & Sigma, Taufkirchen, Germany \\
\hline Glycerol & Roth, Karlsruhe, Germany \\
\hline Glycine & AppliChem, Darmstadt, Germany \\
\hline Hanks Balanced Salt Solution (HBSS) & Gibco, Karlsruhe, Germany \\
\hline HEPES & AppliChem, Darmstadt, Germany \\
\hline Hydrogen peroxide $\left(\mathrm{H}_{2} \mathrm{O}_{2}\right)$ & Merck, Darmstadt, Germany \\
\hline Insulin & Sigma, Taufkirchen, Germany \\
\hline Laminin & Sigma, Taufkirchen, Germany \\
\hline L-ascorbic acid & Sigma, Taufkirchen, Germany \\
\hline LB agar & AppliChem, Darmstadt, Germany \\
\hline LB medium & AppliChem, Darmstadt, Germany \\
\hline L-glutamine & Seromed, Berlin. Germany \\
\hline Luminol & Sigma, Taufkirchen, Germany \\
\hline Magnesium chloride $\left(\mathrm{MgCl}_{2}\right)$ & AppliChem, Darmstadt, Germany \\
\hline Methanol & AppliChem, Darmstadt, Germany \\
\hline Milk powder & Roth, Karlsruhe, Germany \\
\hline Mowiol & CalbioChem, La Jolla, CA, USA \\
\hline N1-supplement & Sigma, Taufkirchen, Germany \\
\hline $\mathrm{N}$-acetyl cysteine & Sigma, Taufkirchen, Germany \\
\hline Neurobasal medium Opti-MEM & Gibco, Karlsruhe, Germany \\
\hline New born goat serum (NGS) & PAA, Coelbe, Germany \\
\hline N-propyl-gallate & Sigma, Taufkirchen, Germany \\
\hline Papain & Cell Signaling, Frankfurt, Germany \\
\hline Paraformaldehyde (PFA) & AppliChem, Darmstadt, Germany \\
\hline PBS (1x Dulbecco's, powder) & AppliChem, Darmstadt, Germany \\
\hline Penicillin/streptomycin/neomycin (PSN) & PAA, Coelbe, Germany \\
\hline Poly-L-ornithine & Sigma, Taufkirchen, Germany \\
\hline Potassium chloride $(\mathrm{KCl})$ & AppliChem, Darmstadt, Germany \\
\hline Progesterone & Sigma, Taufkirchen, Germany \\
\hline Puromycin & Sigma, Taufkirchen, Germany \\
\hline Putrescine & Sigma, Taufkirchen, Germany \\
\hline Raf-1 kinase inhibitor I in solution & CalbioChem, La Jolla, CA, USA \\
\hline Rotiphorese & Roth, Karlsruhe, Germany \\
\hline Sodium acetate & Fluka, Taufkirchen, Germany \\
\hline Sodium bicarbonate $\left(\mathrm{Na}_{2} \mathrm{CO}_{3}\right)$ & Sigma, Taufkirchen, Germany \\
\hline Sodium chloride $(\mathrm{NaCl})$ & AppliChem, Darmstadt, Germany \\
\hline Sodium citrate & CalbioChem, La Jolla, CA, USA \\
\hline Sodium dodecylsulphate (SDS) & AppliChem, Darmstadt, Germany \\
\hline Sodium pyruvate & Gibco, Karlsruhe, Germany \\
\hline Sodium selenite & Sigma, Taufkirchen, Germany \\
\hline $\begin{array}{l}\text { N, N, N', N'-tetrametylethylene diamine } \\
\text { (TEMED) }\end{array}$ & Sigma, Taufkirchen, Germany \\
\hline Transferrin & Sigma, Taufkirchen, Germany \\
\hline Triiodothyronine & Sigma, Taufkirchen, Germany \\
\hline Tris-base & AppliChem, Darmstadt, Germany \\
\hline Triton $\mathrm{X}-100$ & Sigma, Taufkirchen, Germany \\
\hline Trypsin & Sigma, Taufkirchen, Germany \\
\hline Trypsin inhibitor & Boerhinger, Mannheim, Germany \\
\hline
\end{tabular}




\begin{tabular}{ll}
\hline Tryptone & Sigma, Taufkirchen, Germany \\
Tween 20 & Fluka, Taufkirchen, Germany \\
Yeast extract & Sigma, Taufkirchen, Germany \\
\hline
\end{tabular}

Table 1: $\quad$ List of reagents used in this study.

\subsubsection{Consumables}

Consumables were purchased from Sarstedt (Nümbrecht, Germany) if not mentioned otherwise in the text.

\subsubsection{Enzymes and Restriction enzymes}

All enzymes (Klenow polymerase, T4 DNA ligase) and restriction enzymes (NotI, EcoRV, SpeI, ClaI, NheI, SmaI) were purchased from New England BioLabs (Frankfurt/Main, Germany).

\subsubsection{Plasmids}

\subsubsection{1 pFlag-BAG1}

The plasmid pFlag-BAG1 (kindly provided by Dr. Kermer) contains the small isoform (29 $\mathrm{kDa}$ ) of the mouse BAG1 cDNA N-terminally tagged with a Flag epitope under control of the neuron-specific enolase promoter (NSE). Plasmid structure is depicted in Figure 8. The small BAG1 isoform was used because of its high expression in brain.

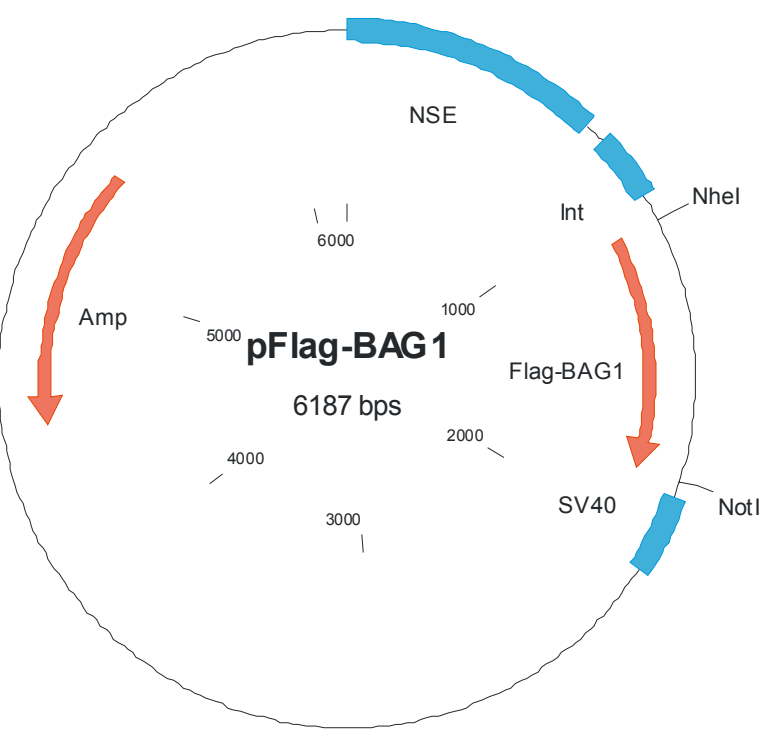

Figure 8: $\quad$ Schematic depiction of the plasmid pFlag-BAG1. Restriction sites used for cloning (NheI, NotI) are indicated on the external side of the plasmid. NSE, neuron-specific enolase promoter; Int, intron; Flag-BAG1, mouse BAG1 cDNA N-terminally tagged with the Flag epitope; SV40, simian virus 40 polyadenylation site; Amp, ampicilline resistance site. 


\subsubsection{2 pAAV-6P1-TB}

The plasmid pAAV-6P1-TB (kindly provided by Dr. Kügler) is a vector containing an AAV bicistronic expression cassette constituted of a Bcl- $\mathrm{X}_{\mathrm{L}}$ cDNA and enhanced green fluorescence protein (EGFP) reporter gene under the control of two independent human synapsin 1 gene promoters (Kügler et al., 2003b), as shown in Figure 9.

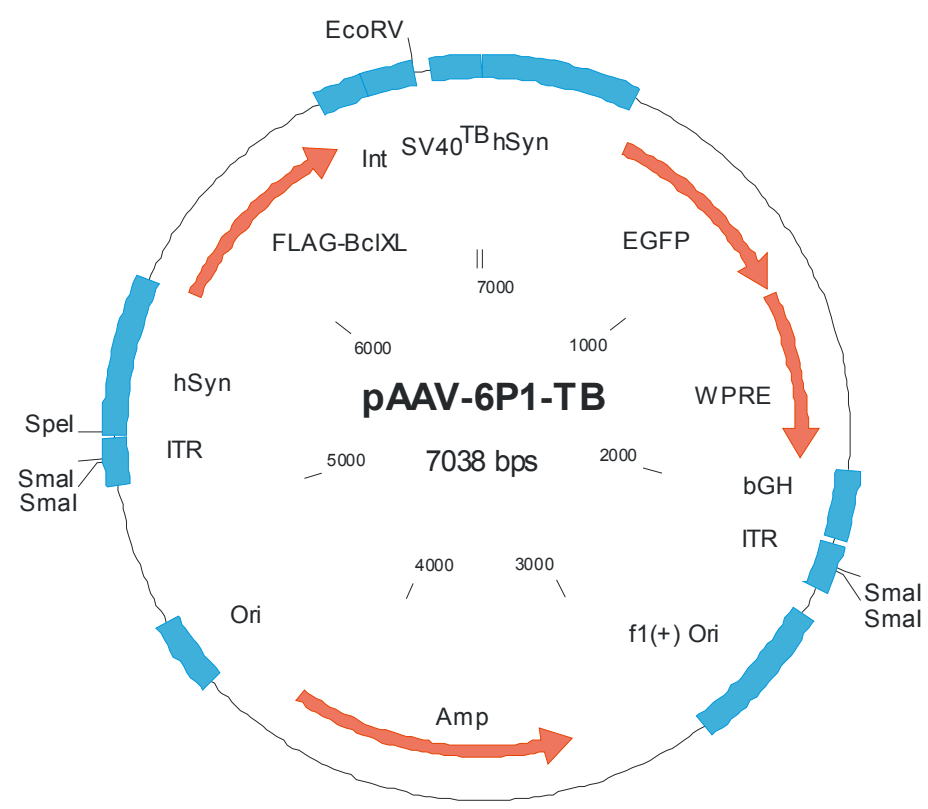

Figure 9: $\quad$ Schematic depiction of the plasmid pAAV-6P1-TB. Restriction sites used for cloning are indicated on the external side of the plasmid. ITR, AAV-2 inverted terminal repeats; hSyn, human synapsin 1 gene promoter; FLAG-Bcl-X $\mathrm{L}_{\mathrm{L}}$, rat Bcl- $\mathrm{X}_{\mathrm{L}}$ cDNA N-terminally tagged with the Flag epitope; Int, intron; SV40, simian virus 40 polyadenylation site; TB, synthetic transcription blocker; EGFP, enhanced green fluorescent protein reporter gene; WPRE, woodchuck hepatitis virus posttranscriptional regulatory element; bGH, bovine growth hormone polyadenylation site; Ori, origin of replication; f1(+) Ori, origin of single strand-DNA replication.

The expression cassette is flanked by two inverted terminal repeats (ITR) of the AAV-2 genome ensuring virus replication. Additional elements required for proper gene expression include a small chimerical intron and the simian virus 40 (SV40) polyadenylation site, the woodchuck hepatitis virus posttranslational control element (WPRE) and the bovine growth hormone (bGH) polyadenylation site.

\subsubsection{3 pBlueScript II KS}

The subcloning vector pBlueScript II KS was purchased from Stratagene (Heidelberg, Germany). Plasmid structure is depicted in Figure 10. 


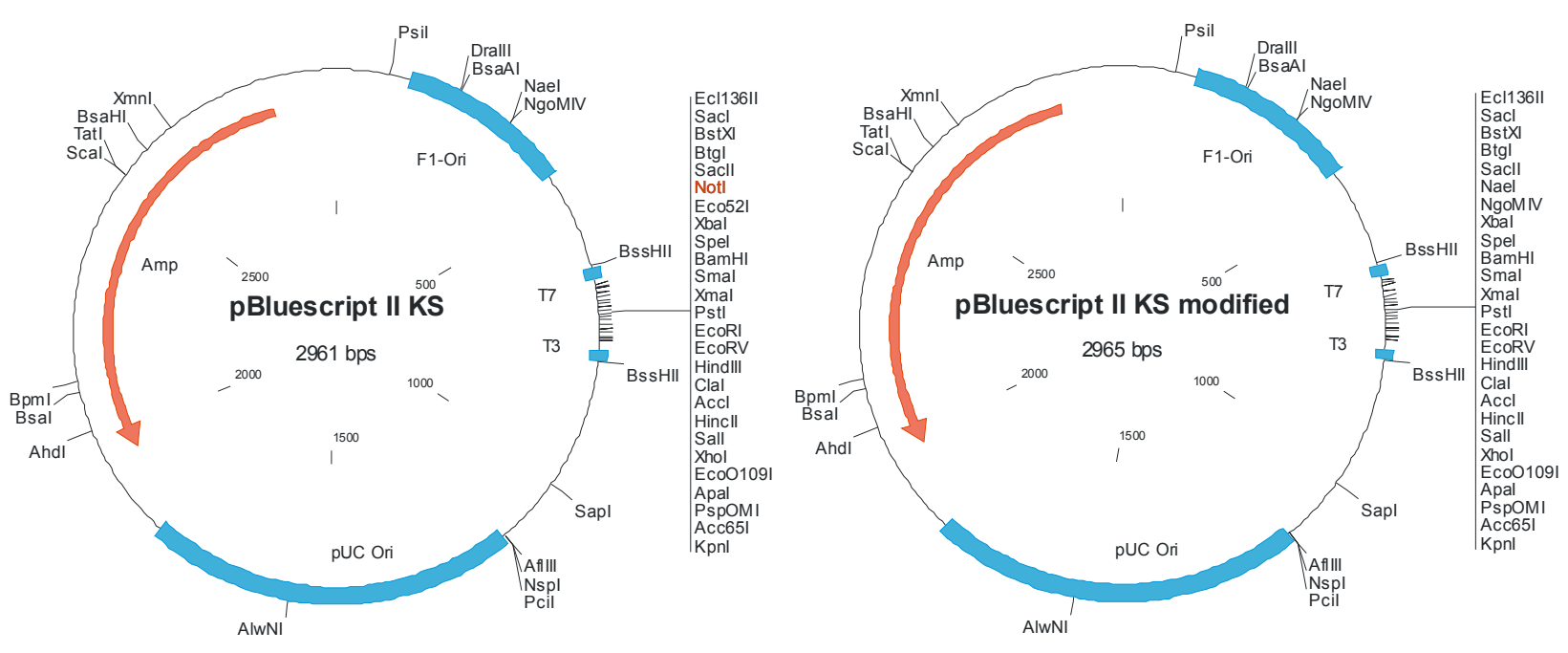

Figure 10: $\quad$ Schematic depiction of the subcloning vectors pBlueScript II KS. For cloning purpose, the Not I restriction site (in red) was removed by restriction digest (see section 2.2.1.9). Amp, resistance to ampicilline; F1-Ori, origin of single strand-DNA replication; T7 and T3, respective promoter transcription initiation sites flanking the multiple cloning site; pUC Ori, origin of replication.

\subsubsection{Cell lines and electrocompetent cells}

Wild-type CSM14.1 and BAG1 stably transfected CSM14.1 cell lines were kindly provided by Dr. Kermer. Escherichia coli $\mathrm{DH} 5 \alpha$ original stock was purchased from Invitrogene (Karlsruhe, Germany).

\subsubsection{Antibodies}

Primary antibodies used in this study are listed in Table 2. Secondary antibodies for immunofluorescence were Cy2, Cy3 or Cy5-coupled anti-mouse, anti-rabbit or anti-goat IgGs (Dianova, Hamburg, Germany). HRP-conjugated anti- mouse, anti-rabbit or anti-goat IgGs (Santa Cruz Biotechnology, Heidelberg, Germany) were used for western blots.

\begin{tabular}{|c|c|c|}
\hline Antibody & Source & Reference \\
\hline Anti-BAG1 & Rabbit & $\begin{array}{l}\text { Kindly provided by Dr. Kermer (Kermer et } \\
\text { al., 2002) }\end{array}$ \\
\hline Anti-Flag M2 & Mouse monocl. & Stratagene, Heidelberg, Germany \\
\hline Anti-GAP43 & Rabbit polycl. & Biozol, Eching, Germany \\
\hline Anti-Gapdh & Mouse monocl. & BioTrend, Cologne, Germany \\
\hline Anti-Mouse IgM ( $\mu$ chain) & Goat monocl. & Biomol, Hamburg, Germany \\
\hline Anti-p44/42 MAPK & Rabbit polycl. & Cell Signaling, Frankfurt/Main, Germany \\
\hline Anti-phospho-ADF-cofilin & Rabbit & $\begin{array}{l}\text { Kind gift of Dr. Bamburg (Colorado State } \\
\text { University, CO, USA, Meberg and } \\
\text { Bamburg, 2000) }\end{array}$ \\
\hline $\begin{array}{l}\text { Anti-phospho-c-Raf } \\
\text { (Ser338) }\end{array}$ & Rabbit monocl. & Cell Signaling, Frankfurt/Main, Germany \\
\hline
\end{tabular}




\begin{tabular}{|c|c|c|}
\hline $\begin{array}{l}\text { Anti-Phospho-p44/42 } \\
\text { MAPK (Thr202/Tyr204) }\end{array}$ & Rabbit monocl. & Cell Signaling, Frankfurt/Main, Germany \\
\hline $\begin{array}{l}\text { Anti-PhosphoSAPK/JNK } \\
\text { (Thr183/Tyr185) }\end{array}$ & Mouse monocl. & Cell Signaling, Frankfurt/Main, Germany \\
\hline Anti-Rabbit IgG $(\mathrm{H}+\mathrm{L})$ & Goat polycl. & Dianova, Hamburg, Germany \\
\hline Anti-Raf-1 & Mouse monocl. & Santa Cruz Biotech., Heidelberg, Germany \\
\hline Anti-rat Macrophage & Rabbit polycl. & Wak-Chemie, Steinbach, Germany \\
\hline Anti-RhoA & Mouse monocl. & Santa Cruz Biotech., Heidelberg, Germany \\
\hline Anti-ROCK2 & Goat polycl. & Santa Cruz Biotech., Heidelberg, Germany \\
\hline Anti- $\beta$-tubulin & Mouse monocl. & Sigma, Taufkirchen, Germany \\
\hline $\begin{array}{l}\text { Rhodamine-conjugated } \\
\text { Phalloidin }\end{array}$ & & Molecular Probes, Karlsruhe, Germany \\
\hline
\end{tabular}

Table 2: $\quad$ List of primary antibodies used in this study. Monocl., monoclonal; polycl., polyclonal.

\subsubsection{Kits and Assays}

\begin{tabular}{ll}
\hline Kits and Assays & Reference \\
\hline Qiagen Plasmid Maxi kit & Qiagen, Hilden, Germany \\
QIAprep spin MiniPrep kit & Qiagen, Hilden, Germany \\
QIAquick Gel extraction kit & Qiagen, Hilden, Germany \\
ROCK kinase activity assay CycLex & MoBiTec, Göttingen, Germany \\
ProteoExtract ${ }^{\circledR}$ Subcellular Proteome & Merck, Darmstadt, Germany \\
Extraction Kit & \\
\hline
\end{tabular}

Table 3: $\quad$ List of kits used in this study.

\subsubsection{Apparatus}

\begin{tabular}{ll}
\hline Instrument & Reference \\
\hline ÄKTA fast protein liquid chromatography & Amersham Biosciences, Freiburg, Germany \\
Apotome $^{\mathrm{T}}$ device & Zeiss, Göttingen, Germany \\
Autoclave & Systec, Greiz, Germany \\
Binocular microscope & World Precision Instruments, Berlin, \\
& Germany \\
Biophotometer & Eppendorf, Hamburg, Germany \\
CCD camera AxioCam & Zeiss, Göttingen, Germany \\
$\mathrm{Cell}_{\text {culture hood, LaminAir }}{ }^{\circledR}$ HB2448 & Heraeus, Hanau, Germany \\
$\mathrm{CO}_{2}$ chamber (for live imaging) & Zeiss, Göttingen, Germany \\
$\mathrm{Confocal} \mathrm{microscope} \mathrm{AOBS}$ & Leica, Nussloch, Germany \\
Cryostat $_{\text {CTI-controller (for live imaging) }}$ & Leica, Nussloch, Germany \\
Electrophoresis chambers (for DNA) & Zeiss, Göttingen, Germany \\
Electrophoresis chambers (for Western blot) & BioRad, Munich, Germany \\
Electrophoresis power supply & BioRad, Munich, Germany \\
ELISA spectrophotometer, Tecan RainBow & Tecan, Crailsheim, Germany \\
Fluorescence microscope, Axioplan & Zeiss, Göttingen, Germany \\
Fluorescence microscope, Axiovert & Zeiss, Göttingen, Germany \\
Fluor-S ${ }^{\mathrm{TM}}$-MultiImager & Bio-Rad, Munich, Germany \\
\hline
\end{tabular}




\begin{tabular}{ll}
\hline Freezers & Heraeus, Hanau, Germany \\
Gel Documentation Transluminator & Bio-Rad, Munich, Germany \\
Heat block, ThermoStat Plus & Eppendorf, Hamburg, Germany \\
Ice machine, Scotman ${ }^{\circledR}$ & Frimont, Milan, Italy \\
Incubator shaker & Schütt, Göttingen, Germany \\
Incubator, for bacterial culture & Heraeus, Hanau, Germany \\
Incubator, for cell culture & B. Braun, Melsungen, Germany \\
Instruments for dissection & Fine Science Tools, Heidelberg, Germany \\
Light microscope, Axiovert 25 & Zeiss, Göttingen, Germany \\
Microwave oven & Bosch, Stuttgart, Germany \\
Operating microscope & Zeiss, Göttingen, Germany \\
pH-meter & Sartorius, Göttingen, Germany \\
Pipettes & Gilson, Villiers le Bel, France \\
Pipetting device, Accu-Jet & Brand, Wethiem, Germany \\
Refrigerators & Liebherr, Biberach, Germany \\
Scales & Sartorius, Göttingen, Germany \\
Sonicator & Bandelin, Berlin, Germany \\
Sterilisator & Fine Science Tools, Heidelberg, Germany \\
Table centrifuges & Eppendorf, Hamburg, Germany; Heraeus, \\
& Hanau, Germany \\
Temperature-controller (for live imaging) & Zeiss, Göttingen, Germany \\
Ultracentrifuge, Sorvall & DuPont Instruments, Bag Homburg, \\
& Germany \\
Vortex mixer & NeoLab, Heidelberg, Germany \\
Waterbath & GFL, Burgwedel, Germany \\
Water purifier, PureLab Plus & Elga Labwaters, Ransbach-Baumbach, \\
& Germany \\
\hline & \\
\hline
\end{tabular}

Table 4: $\quad$ List of instruments used in this study.

\subsection{Media, buffers, and solutions}

Protocols mention the suitable volumes to perform the experiments described in the following chapters. If not mentioned otherwise, solutions were prepared with distilled and UV-purified RNase-free water (indicated as $\mathrm{H}_{2} \mathrm{O}_{\text {dist. }}$ ). Reagents, buffers and solutions are listed in alphabetical order in the respective sections. Final concentrations are given in brackets.

\subsubsection{Reagents in solution}

\section{Complete protease inhibitor}

Two tablets were dissolved in $840 \mu \mathrm{H}_{2} \mathrm{O}_{\text {dist. }}(8.8 \mathrm{mg} / \mathrm{ml})$.

\section{DEPC water}

$500 \mu \mathrm{l}$ of DEPC were added to $1000 \mathrm{ml} \mathrm{H}_{2} \mathrm{O}_{\text {dist. }}$ and well mixed. Solution was incubated overnight at $37^{\circ} \mathrm{C}$ under constant stirring and autoclaved before use. 


\section{DNase}

$5 \mathrm{mg}$ of DNase were dissolved in $1 \mathrm{ml}$ of $\mathrm{Ca}^{2+} / \mathrm{Mg}^{2+}$-Free (CMF) medium and stored at $-20^{\circ} \mathrm{C}$ $(5 \mathrm{mg} / \mathrm{ml})$.

\section{FCS (inactivated)}

FCS was pre-warmed at $37^{\circ} \mathrm{C}$ and incubated for $30 \mathrm{~min}$ at $56^{\circ} \mathrm{C}$ (heat inactivation). Aliquots were stored at $-20^{\circ} \mathrm{C}$.

Forskolin $1000 x$

$2.4 \mathrm{ml}$ of DMSO were added to $10 \mathrm{mg}$ of Forskolin $(4.2 \mathrm{mg} / \mathrm{ml})$.

\section{Glycine}

$3.75 \mathrm{~g}$ of glycine were dissolved in $500 \mathrm{ml} \mathrm{H} \mathrm{O}_{\text {dist. }}(100 \mathrm{mM})$ and $\mathrm{pH}$ was adjusted to 2.9.

\section{Insulin}

$0.0125 \mathrm{mg}$ of insulin was dissolved in $5 \mathrm{ml}$ of DMEM-F12, and solution was sterile filtered. Aliquots were stored at $4^{\circ} \mathrm{C}$ in darkness.

\section{Laminin}

Laminin was diluted 1:1000 in DMEM-F12 before use.

\section{Na pyruvate}

$5.5 \mathrm{mg}$ of Na pyruvate were dissolved in $5 \mathrm{ml}$ of PBS. The solution was sterile filtered and aliquots were stored at $-20^{\circ} \mathrm{C}$.

$N$-acetyl cysteine $1000 x$

$50 \mathrm{mg}$ of $\mathrm{N}$-acetyl cysteine were dissolved in $10 \mathrm{ml}$ of Neurobasal medium $(5 \mathrm{mg} / \mathrm{ml})$.

\section{Poly-D-ornithine}

$1 \mathrm{mg}$ of poly-D-ornithine was dissolved in $1 \mathrm{ml}$ of sterile borate buffer $(1 \mathrm{mg} / \mathrm{ml})$. 
Sato stock $100 x$

\begin{tabular}{ll}
\hline Transferrin & $100 \mu \mathrm{g} / \mathrm{ml}$ \\
BSA & $100 \mu \mathrm{g} / \mathrm{ml}$ \\
Progesterone & $60 \mu \mathrm{g} / \mathrm{ml}$ \\
Putrescine & $16 \mu \mathrm{g} / \mathrm{ml}$ \\
Na selenite & $40 \mathrm{ng} / \mathrm{ml}$ \\
in Neurobasal medium & \\
Sterile filtered & \\
\hline
\end{tabular}

T3 $100 x$

$3.2 \mathrm{mg}$ of triiodothyronine were dissolved in $400 \mathrm{ml}$ of $0.1 \mathrm{~N} \mathrm{NaOH} .10 \mathrm{ml}$ of the obtained solution were added to $20 \mathrm{ml}$ of DPBS and the solution was sterile filtered.

Trypsin $0.25 \%$

$25 \mathrm{mg}$ of trypsin were dissolved in $10 \mathrm{ml} \mathrm{CMF} \mathrm{medium} \mathrm{and} \mathrm{the} \mathrm{solution} \mathrm{was} \mathrm{sterile} \mathrm{filtered.}$

\subsubsection{Bacteria culture medium}

\section{$L B$ medium}

$25 \mathrm{~g}$ of LB powder were dissolved in $1000 \mathrm{ml} \mathrm{H}_{2} \mathrm{O}_{\text {dist. }} \mathrm{pH}$ was adjusted to 7.0 and the solution autoclaved.

\section{LB agar}

$11.2 \mathrm{~g}$ of LB agar were dissolved in $350 \mathrm{ml} \mathrm{H} \mathrm{O}_{\text {dist. }}$ and the solution was autoclaved. The solution was cooled to $60^{\circ} \mathrm{C}$ and the appropriate resistance antibiotic $(10 \mathrm{mg} / \mathrm{ml})$ was added shortly before use. $18 \mathrm{ml}$ agar were poured per Petri dish and let dry overnight. Plates were stored at $4^{\circ} \mathrm{C}$.

SOB medium (Hanahan, 1983)

\begin{tabular}{ll}
\hline Tryptone & $20 \mathrm{~g} / \mathrm{l}$ \\
Yeast extract & $5 \mathrm{~g} / 1$ \\
$\mathrm{NaCl}$ & $10 \mathrm{mM}$ \\
$\mathrm{KCl}$ & $2.5 \mathrm{mM}$ \\
$\mathrm{MgCl}_{2}$ & $10 \mathrm{mM}$ \\
$\mathrm{MgSO}_{4}$ & $10 \mathrm{mM}$ \\
in $\mathrm{H}_{2} \mathrm{O}_{\text {dist. }}$ & \\
\hline
\end{tabular}




\section{SOC medium}

$1 \mathrm{ml}$ of Glucose was added to $100 \mathrm{ml}$ of SOB medium $(20 \mathrm{mM})$ and the solution was then stored at $4^{\circ} \mathrm{C}$.

\subsubsection{Cell culture media}

$\mathrm{Ca}^{2+} / \mathrm{Mg}^{2+}$-Free (CMF) medium

$50 \mathrm{ml}$ of 10x Hanks Balanced Salt Solution (HBSS) were added to $450 \mathrm{ml}$ sterile $\mathrm{H}_{2} \mathrm{O}_{\text {dist. }}$ (final dilution $1 \mathrm{x}$ ). $\mathrm{pH}$ was adjusted to 7.2 with sterile $\mathrm{Na}$ bicarbonate.

\section{$D M E M$}

Dulbecco's modified Eagle's medium, used as supplied by manufacturer.

$D P B S$

Dulbecco's Phosphate Buffered Saline, used as supplied by manufacturer.

\section{EBSS}

Earle's Buffered Salt Solution, used as supplied by manufacturer.

\section{High Ovomucoid $6 x$}

\begin{tabular}{ll}
\hline BSA & $60 \mathrm{mg} / \mathrm{ml}$ \\
Trypsin inhibitor & $60 \mathrm{mg} / \mathrm{ml}$ \\
in DPBS & \\
pH adjusted to & 7.4 \\
Sterile filtered. & \\
\hline
\end{tabular}

Low Ovomucoid 10x

\begin{tabular}{ll}
\hline BSA & $15 \mathrm{mg} / \mathrm{ml}$ \\
Trypsin inhibitor & $15 \mathrm{mg} / \mathrm{ml}$ \\
in DPBS & \\
pH adjusted to & 7.4 \\
Sterile filtered. & \\
\hline
\end{tabular}

Neurobasal medium (HC)

\begin{tabular}{lrl}
\hline Transferrin & $5 \mu \mathrm{g} / \mathrm{ml}$ \\
PSN containing : & Penicillin & $50 \mu \mathrm{g} / \mathrm{ml}$ \\
& Streptomycin & $50 \mu \mathrm{g} / \mathrm{ml}$ \\
\hline
\end{tabular}




\begin{tabular}{lll}
\hline & Neomycin & $100 \mu \mathrm{g} / \mathrm{ml}$ \\
Glutamine & $2 \mathrm{mM}$ \\
B-27 supplement & $2 \% \mathrm{vol}$. \\
in Neurobasal medium & \\
\hline
\end{tabular}

Neurobasal SATO

\begin{tabular}{lrl}
\hline PSN containing: & $\begin{array}{r}\text { Penicillin } \\
\text { Streptomycin }\end{array}$ & $50 \mu \mathrm{g} / \mathrm{ml}$ \\
& $50 \mu \mathrm{g} / \mathrm{ml}$ \\
Neomycin & $100 \mu \mathrm{g} / \mathrm{ml}$ \\
Insulin & $25 \mathrm{ng} / \mathrm{ml}$ \\
Na pyruvate & $11 \mu \mathrm{g} / \mathrm{ml}$ \\
Sato stock 100x & $1 \% \mathrm{vol}$. \\
T3 100x & $1: 100$ \\
Glutamine & $2 \mu \mathrm{mM}$ \\
N-acetyl cysteine & $50 \mu \mathrm{g} / \mathrm{ml}$ \\
B-27 supplement 100x & $2 \% \mathrm{vol}$. \\
BDNF & $50 \mu \mathrm{g} / \mathrm{ml}$ \\
CNTF & $10 \mu \mathrm{g} / \mathrm{ml}$ \\
Forskolin & $10 \mu \mathrm{g} / \mathrm{ml}$ \\
in Neurobasal medium & \\
\hline
\end{tabular}

$R G C$ medium

\begin{tabular}{lrl}
\hline FCS & $10 \% \mathrm{vol}$ \\
PSN containing : & $\begin{array}{r}\text { Penicillin } \\
\text { Streptomycin } \\
\text { Neomycin }\end{array}$ & $50 \mu \mathrm{g} / \mathrm{ml}$ \\
& $100 \mu \mathrm{g} / \mathrm{ml}$ \\
& $2 \mathrm{mM}$ \\
Glutamine & \\
in DMEM-F12 & & \\
\hline
\end{tabular}

\subsubsection{Buffers and solutions}

\section{Borate buffer}

$0.928 \mathrm{~g}$ of borate was dissolved in $100 \mathrm{ml} \mathrm{H}_{2} \mathrm{O}_{\text {dist. }}(150 \mathrm{mM})$ and $\mathrm{pH}$ was adjusted to 8.4 . The solution was sterile filtered.

$\mathrm{CaCl}_{2} 50 \mathrm{mM}$

$3.7 \mathrm{~g}$ of $\mathrm{CaCl}_{2}$ were dissolved in $500 \mathrm{ml}$ of $\mathrm{H}_{2} \mathrm{O}_{\text {dist. }}$.

$\mathrm{CaCl}_{2} 50 \mathrm{mM} / 15 \%$ Glycerine

$7.5 \mathrm{ml}$ of glycerine were added to $50 \mathrm{ml}$ of $\mathrm{CaCl}_{2} 50 \mathrm{mM}$ solution. 


\section{Chloral hydrate $7 \%$}

$7 \mathrm{~g}$ of chloral hydrate were dissolved in $100 \mathrm{ml}$ of $\mathrm{H}_{2} \mathrm{O}_{\text {dist. }}$ and the solution was sterile filtered.

\section{DNA loading buffer $5 x$}

For $20 \mathrm{ml}$ solution, $3 \mathrm{~g}$ of Ficoll $400 \mathrm{DL}$ (15\%), $84 \mathrm{mg}$ of $\mathrm{LiCl}(100 \mathrm{mM}), 744.5 \mathrm{mg}$ of EDTA $(100 \mathrm{mM})$ and $10 \mathrm{mg}$ of bromophenol blue $(0.05 \%)$ were dissolved in DEPC water.

$E C L$

\begin{tabular}{lll}
\hline Solution 1: & Luminol & $2.5 \mathrm{mM}$ \\
& p-Coumaric acid & $0.4 \mathrm{mM}$ \\
& Tris pH 8.5 & $0.1 \mathrm{M}$ \\
& in $\mathrm{H}_{2} \mathrm{O}_{\text {dist. }}$ & \\
Solution 2: & $\mathrm{H}_{2} \mathrm{O}_{2}$ & $18 \%$ \\
& Tris pH 8.5 & $0.1 \mathrm{M}$ \\
& in $\mathrm{H}_{2} \mathrm{O}_{\text {dist. }}$ & \\
\hline
\end{tabular}

Both solutions were mixed in 1:1 ratio shortly before use.

\section{Electrophoresis buffer}

$30.24 \mathrm{~g}$ of Tris $(25 \mathrm{mM}), 142.5 \mathrm{~g}$ of glycine $(192 \mathrm{mM})$ and $10 \mathrm{~g}$ of SDS $(0.1 \%)$ were dissolved in $1000 \mathrm{ml} \mathrm{H}_{2} \mathrm{O}_{\text {dist. }}$ and $\mathrm{pH}$ was adjusted to 8.3.

\section{$\mathrm{HCl} 1 \mathrm{M}$}

$20 \mathrm{ml}$ of $\mathrm{HCl} 37 \%$ were added to $180 \mathrm{ml} \mathrm{H}_{2} \mathrm{O}_{\text {dist. }}$

HEPES-buffer $25 \mathrm{mM}$, $\mathrm{pH} 7.5$

$5.957 \mathrm{~g}$ of HEPES were added into $1000 \mathrm{ml}$ of $\mathrm{H}_{2} \mathrm{O}_{\text {dist. }}$ and $\mathrm{pH}$ was adjusted to 7.5.

\section{Mowiol}

$5 \mathrm{~g}$ of mowiol were dissolved in $20 \mathrm{ml}$ PBS under constant stirring for $4 \mathrm{~h}$ at $4^{\circ} \mathrm{C} .10 \mathrm{ml}$ of glycerine were added and dissolved under shaking for $16 \mathrm{~h}$ at $4{ }^{\circ} \mathrm{C}$. After centrifugation for 15 min at $4000 \mathrm{rpm}(1699 \mathrm{~g})$, remove supernatant and carefully adjust $\mathrm{pH}$ to $8.0 .0 .3 \mathrm{ml}$ of $\mathrm{Na}$ azide ( $2 \%$ vol.) and a tip of N-propyl-gallate were added into $10 \mathrm{ml}$ and the solution was centrifuged $20 \mathrm{~min}$ at $4000 \mathrm{rpm}(1699 \mathrm{~g})$. 


\section{Paraformaldehyde (PFA) 4\%}

$40 \mathrm{~g}$ of PFA were dissolved in $700 \mathrm{ml}$ of filtered PBS under heating $\left(55^{\circ} \mathrm{C}\right)$. $\mathrm{pH}$ was carefully adjusted to 7.4 and the solution filled up to $1000 \mathrm{ml}$ with PBS.

\section{$P B S$}

$9.55 \mathrm{~g}$ of PBS powder were dissolved in $1000 \mathrm{ml} \mathrm{H}_{2} \mathrm{O}_{\text {dist. }}$ For preparation of solutions to be used in vivo, PBS solution was sterile filtered.

SDS protein loading buffer $5 x$

$0.756 \mathrm{~g}$ of Tris base was dissolved in $5 \mathrm{ml} \mathrm{H}_{2} \mathrm{O}_{\text {dist. }}$ and $\mathrm{pH}$ was carefully adjusted to 6.8. $2 \mathrm{~g}$ of SDS (SDS 2x cryst. analytical grade, Serva, Heidelberg, Germany), $0.001 \mathrm{~g}$ of bromophenol blue and $10 \mathrm{ml}$ glycerine were added in warm Tris and the solution was filled up to $20 \mathrm{ml}$ with $\mathrm{H}_{2} \mathrm{O}_{\text {dist. }} 10 \% \beta$-mercaptoethanol was added before use.

\section{Transfer buffer}

\begin{tabular}{ll}
\hline $\mathrm{HEPES}(0.6 \mathrm{~g} / 250 \mathrm{ml})$ & $10 \mathrm{mM}$ \\
$\mathrm{CaCl}_{2} 2 \mathrm{H}_{2} \mathrm{O}$ & $15 \mathrm{mM}$ \\
$\mathrm{KCl}$ & $250 \mathrm{mM}$ \\
$\mathrm{H}_{2} \mathrm{O}_{\text {dist. }}$ & \\
$\mathrm{pH}$ adjusted to & 6.7 \\
$\mathrm{MnCl}_{2} 2 \mathrm{H}_{2} \mathrm{O}$ & $55 \mathrm{mM}$ \\
sterile filtered and stored at $4^{\circ} \mathrm{C}$. & \\
\hline
\end{tabular}

Tris-Borate-EDTA (TBE) buffer

$108 \mathrm{~g}$ of Tris-base $(50 \mathrm{mM}), 55 \mathrm{~g}$ of borate $(42 \mathrm{mM})$, and $9.3 \mathrm{~g}$ of EDTA $(10 \mathrm{mM})$ were dissolved in $\mathrm{H}_{2} \mathrm{O}_{\text {dist. }} \mathrm{pH}$ was adjusted to 8.0 and the solution was autoclaved.

\section{TBS $10 x$ for antigen retrieval}

$80 \mathrm{~g}$ of $\mathrm{NaCl}(150 \mathrm{mM})$ and $24.2 \mathrm{~g}$ of Tris base $(10 \mathrm{mM})$ were dissolved in $1000 \mathrm{ml} \mathrm{H}_{2} \mathrm{O}_{\text {dist. }}$ The $\mathrm{pH}$ of $1 \mathrm{x}$ TBS solution was adjusted to 9.0 .

\section{TBS lysis buffer}

$100 \mu 1$ of Complete Protease Inhibitor (4\%) and $12.5 \mu 1$ of Triton-X 100 (1\%) were dissolved in $2.5 \mathrm{ml}$ of TBS. 
$T B S-T$

$10 \mathrm{ml}$ of Tween $20(0.1 \%), 80 \mathrm{~g}$ of $\mathrm{NaCl}(150 \mathrm{mM})$ and $24.2 \mathrm{~g}$ of Tris-base $(10 \mathrm{mM})$ were dissolved in $\mathrm{H}_{2} \mathrm{O}_{\text {dist. }}$ and $\mathrm{pH}$ was adjusted to 7.6.

\section{Transfer buffer $1 x$}

$3.025 \mathrm{~g}$ of Tris-base $(25 \mathrm{mM}), 14.4 \mathrm{~g}$ of glycine $(192 \mathrm{mM})$ and $200 \mathrm{ml}$ of methanol $(20 \%)$ were dissolved in $\mathrm{H}_{2} \mathrm{O}_{\text {dist. }}$ and $\mathrm{pH}$ was adjusted to 8.3.

\section{Tris/SDS pH $6.84 x$}

$6.05 \mathrm{~g}$ of Tris-base $(0.5 \mathrm{M})$ and $0.4 \mathrm{~g}$ of SDS $(0.4 \%)$ were dissolved in $100 \mathrm{ml}$ of $\mathrm{H}_{2} \mathrm{O}_{\text {dist. }}$ and $\mathrm{pH}$ was adjusted to 6.8 .

Tris/SDS pH $8.84 x$

$6.05 \mathrm{~g}$ of Tris-base $(1.5 \mathrm{M})$ and $0.4 \mathrm{~g}$ of SDS $(0.4 \%)$ were dissolved in $100 \mathrm{ml} \mathrm{of} \mathrm{H}_{2} \mathrm{O}_{\text {dist. }}$ and $\mathrm{pH}$ was adjusted to 8.8 .

\subsection{Methods}

\subsubsection{Molecular biology}

\subsubsection{DNA restriction}

Restriction enzymes recognise and cleave a specific DNA sequence. The most commonly used enzymes in laboratory routine recognise a 6- to 8-nucleotide palindrome sequence and generate by cleavage either blunt ends or sticky ends with extensions at 5' or 3'.

For restriction digestion, 160 to $1500 \mu \mathrm{g}$ DNA (for control digestion or generation of fragments for further cloning steps, respectively) were usually used. Restriction enzymes and DNA were mixed in the appropriate buffers (BioLabs) and incubated from 0.5 to $2 \mathrm{~h}$ at the temperature specified for each enzyme according to the manufacturer's instruction manual.

\subsubsection{Agarose gels and electrophoresis}

Agarose gel electrophoresis is a technique allowing the separation of DNA fragments of different sizes and the analysis of DNA size. The gel is formed of a matrix of microscopic fibres whose density depends on the gel percentage. Submitted to an electric field, negatively charged linear DNA fragments migrate to the anode according to their size. Fragments are visualised by exposure to UV light upon addition of ethidium bromide. 
To prepare the gel, 1\% agarose was dissolved in TBE buffer, heated in a microwave oven and $0.3 \mu \mathrm{g} / \mathrm{ml}$ ethidium bromide was added. DNA samples were mixed with $5 \mathrm{x}$ DNA loading buffer (end concentration $1 \mathrm{x}$ ) and DEPC water to obtain an equal final volume of 10 to 12.5 $\mu \mathrm{l}$ before loading the gel. The gel was run in 1x TBE buffer, usually at 100 Volts. The DNA fragments were visualised by UV-light $(302 \mathrm{~nm})$ at a Gel Documentation 2000 ${ }^{\mathrm{TM}} \mathrm{UV}$ transilluminator using the Quantity One software (version 4.2.1).

\subsubsection{Gel extraction}

Isolation of DNA fragments from polyacrylamide gels is based on the method described by Maxam and Gilbert (Maxam and Gilbert, 1977). In the presence of high concentration of salt, DNA adsorbs to a silica membrane while contaminants such as agarose, dyes, ethidium bromide, and detergents are washed away. DNA can then be eluted by addition of a low-salt solution at $\mathrm{pH}$ 7.0-8.5. DNA extraction was performed with a QIAquick Gel Extraction kit following the manufacturer's instruction manual.

\subsubsection{DNA precipitation}

Ethanol precipitation allows DNA concentration and slight purification. Upon addition of a monovalent salt (e.g. sodium acetate, sodium chloride, ammonium acetate) and ethanol to the DNA solution, DNA precipitates spontaneously while salts and soluble substances usually remain in solution. DNA can then be collected by centrifugation and further purified through washing with $70 \%$ ethanol.

One volume of ammonium acetate, 6 volumes of $100 \%$ ethanol and $1 \mu 1$ glycogen were added to one volume of DNA sample solution, vortexed and centrifuged $20 \mathrm{~min}$ at maximal speed in a microcentrifuge. The supernatant was removed, $1 \mathrm{ml}$ of $70 \%$ ethanol added to the pellet and the solution centrifuged 2 more min at the highest speed. The supernatant was then removed, the DNA pellet left until dry at room temperature (RT) and then resuspended in the desired volume of Tris-Cl $10 \mathrm{mM}$ pH 8.5 or DEPC water.

\subsubsection{Determination of DNA concentration}

Calculation of DNA concentration is based on optical density at $260 \mathrm{~nm}$, sample dilution and a specific multiplication factor. Additionally, the ratio $\mathrm{OD}_{260 \mathrm{~nm}} / \mathrm{OD}_{280 \mathrm{~nm}}$ can give an indication on protein contamination in the sample. The DNA concentration was measured by optical density with a Biophotometer at $260 \mathrm{~nm}$. 


\subsubsection{DNA ligation}

DNA ligation allows insertion of cDNA fragments into a plasmid. The T4 ligase catalyses the formation of phosphodiester binding between the 5' phosphorylated end and the 3' nonphosphorylated end of two double strand DNA fragments in presence of ATP. Ligation can occur between blunt ends or compatible sticky ends.

For the ligation, DNA vector and cDNA fragments were mixed in a molar ratio of 1:3 in 10x T4 DNA ligase buffer, DEPC water and $1 \mu \mathrm{T} 4$ ligase to reach a final volume of $20 \mu \mathrm{l}$. The reaction mixture was then left $2 \mathrm{~h}$ at RT and the ligation product subjected to bacterial transformation.

\subsubsection{Production of chemically competent cells and bacterial transformation}

DH5 $\alpha$ competent cells (Escherichia coli, K12 recombination-deficient suppressing strain) were produced as described previously (Inoue et al., 1990). Briefly, $5 \mathrm{ml}$ overnight culture were seeded into $500 \mathrm{ml} \mathrm{LB}$ medium and incubated at $37^{\circ} \mathrm{C}$ under shaking to reach an optic density $\mathrm{OD}_{600}$ of 0.4 to 0.6 . Bacteria were then cooled ca. $20 \mathrm{~min}$ on ice and centrifuged 15 min at $3000 \mathrm{rpm}(0.8 \mathrm{~g})$ at $4^{\circ} \mathrm{C}$. The pellet was resuspended in $250 \mathrm{ml}$ of $50 \mathrm{mM} \mathrm{CaCl}_{2}$, incubated $20 \mathrm{~min}$ on ice and centrifuged again $15 \mathrm{~min}$ at $3000 \mathrm{rpm}(0.8 \mathrm{~g})$ at $4^{\circ} \mathrm{C}$. The pellet was then resuspended in $25 \mathrm{ml} 50 \mathrm{mM} \mathrm{CaCl}_{2} / 15 \%$ glycerine and incubated $60-90 \mathrm{~min}$ on ice. Bacteria were aliquoted $(300 \mu \mathrm{l} /$ tube $)$ and stored at $-80^{\circ} \mathrm{C}$.

Bacteria were transformed by heat shock in calcium chloride. For this, DH5 $\alpha$ competent cells were thawed on ice. 1-5 $\mu 1$ DNA were added to $100 \mu \mathrm{l}$ bacteria on ice and incubated $30 \mathrm{~min}$ on ice. Bacteria were then heat-shocked for $50 \mathrm{sec}$ at $42^{\circ} \mathrm{C}$, and chilled for 2 min on ice. 800 $\mu \mathrm{l}$ warm SOC medium were added and bacteria were incubated for $1 \mathrm{~h}$ at $37^{\circ} \mathrm{C}$ under moderate shaking to allow the bacteria to recover and to express the antibiotic resistance protein encoded by the plasmid. 20 and $100 \mu \mathrm{l}$ of the transformed bacteria were then respectively plated on Petri dishes and incubated for $12 \mathrm{~h}$ at $37^{\circ} \mathrm{C}$. Transformed bacteria expressing the ampicilline resistance protein were selected by culture on LB agar containing the corresponding antibiotic (ampicilline $100 \mu \mathrm{g} / \mathrm{ml}$ ). 


\subsubsection{Plasmid mini- and maxi-preps}

Mini- and maxi-preps allow DNA plasmid extraction on a smaller or larger scale and are both based on the same principle, the alkaline lysis. Plasmids, in contrast to bacterial genomic DNA, are not physically bound to the membrane. To extract them, bacteria are lysed using an alkaline solution constituted of SDS, an ionic denaturing agent, $\mathrm{NaOH}$ and RNase. Addition of potassium acetate and chloroform neutralises $\mathrm{NaOH}$ and precipitates SDS, proteins and genomic DNA associated to proteins. Under appropriate low-salt and $\mathrm{pH}$ conditions, plasmidic DNA is bound to an anion-exchange resin column and eluted in a high-salt buffer. Plasmidic DNA is then concentrated and desalted by isopropanol precipitation.

Mini- and maxi-preps were performed with Qiagen Plasmid Mini and Maxi kit following the manufacturer's instructions.

\subsubsection{Cloning strategy}

\section{AAV.BAG1}

Construction of AAV vectors has been described previously (Kügler et al., 2003b). For this study, we employed an AAV-2 bicistronic vector expressing the functional Flag-BAG1 cDNA and, in a separate cassette, a fluorescent EGFP reporter, both under the control of independent human synapsin 1 gene promoters. To obtain it, we used pAAV-6P1-TB plasmid, the plasmid pFlag-BAG1, and the subcloning vector pBlueScript II KS (for schematic depictions of plasmids, see Figures 8, 9 and 10, section 2.1.4, respectively).

\section{Cloning into subcloning vector}

In order to proceed to the cloning of BAG1 into the AAV expression cassette, a subcloning vector lacking the NotI restriction site was needed. For this purpose, the NotI restriction site present in pBlueScript II KS was deleted by NotI restriction digest. Plasmid ends were refilled using the Klenow polymerase and the vector was religated (modified pBlueScript II KS, Figure 10, section 2.1.4.3). Thus, we obtained a subcloning vector lacking the NotI restriction site. The pAAV-6P1-TB expression cassette containing Bcl- $\mathrm{X}_{\mathrm{L}}$ was extracted using SpeI and EcoRV and inserted into the modified pBlueScript II KS, previously cut with the same restriction enzymes. The pFlag-BAG1 vector was then cut by NheI and NotI and the FlagBAG1 cDNA extracted. Bcl-X $\mathrm{X}_{\mathrm{L}}$ cDNA was then substituted by the cDNA of Flag-BAG1 using the same restriction enzymes. 


\section{Transfer into pAAV backbone}

In order to transfer the expression cassette back into the AAV backbone, the subcloning vector was digested with ClaI at one single site and filled in with Klenow polymerase to obtain blunt ends, which would match the EcoRV blunt ends present in the pAAV backbone. The obtained plasmid was further digested with SpeI. The expression cassette was then cloned back into the pAAV-6P1-TB backbone previously cut with SpeI and EcoRV. The presence of one sticky and one blunt end allowed the insertion of the expression cassette in the right orientation. Thus, we obtained pAAV-BAG1-EGFP. Finally, the integrity of the AAV genome, indispensable for the virus production, was confirmed by digestion with SmaI, which cuts within the ITR regions responsible for the proper replication of the virus. The BAG1 fragment was sent for sequencing to SeqLab laboratories (Göttingen, Germany) to verify the sequence correctness before production of the AAV vectors.

\section{AAV.EGFP}

As a negative control, we used an (AAV-2) AAV.EGFP control vector, the construction of which has been described previously (Malik et al., 2005, kindly provided by Dr. Kügler). This vector expresses EGFP from a hSyn1 promoter but contains a disabled Bcl- $\mathrm{X}_{\mathrm{L}}$ expression cassette constituted of Bcl- $\mathrm{X}_{\mathrm{L}}$ cDNA preceded by a transcription blocker (TB). This presents the advantage of having a vector of the same structure and size as the AAV.BAG1 vector, but expressing only one functional gene (e.g. EGFP reporter gene).

\subsubsection{Viral vector production and purification}

\subsubsection{Virus production}

In order to exclude any adenoviral contamination, viral vectors were propagated in 293 cells using the pDG2 helper, a plasmid encoding the proteins necessary for AAV replication (e.g. capside proteins) (Grimm et al., 1998). Viral particles were purified according to established protocols (Zolotukhin et al., 1999) by iodixanol step gradient centrifugation. Samples were then purified and concentrated by heparin-affinity chromatography on an ÄKTA fast protein liquid chromatography system using $1 \mathrm{ml} \mathrm{HiTrap}{ }^{\mathrm{TM}}$ Heparin QFF columns and desalted overnight by dialysis against PBS.

\subsubsection{Virus titration}

Genome titres were determined by quantitative polymerase chain reaction (PCR) and purity was confirmed by SDS-PAGE. Titre calculations are based on the assumption that vector 
preparations contained a ratio of a 1:30 transducing units versus viral particles. Due to the neuron-specific expression characteristics of the synapsin promoter (Kügler et al., 2001), functional titres of the vectors were confirmed by EGFP expression analysis in cultured hippocampal (HC) neurons (see section 2.3.3.2). Lysates obtained from these neuronal cultures were used to confirm protein expression from the viral vectors by Western blots (see sections 2.3.4.1 and 2.3.4.4).

\subsubsection{Cell culture}

\subsubsection{Coating of culture plates}

In order to create physiologically relevant in vitro models supporting normal cell growth and function, and ensure appropriate cell culture conditions, culture plates can be coated with different attachment factors. In our studies, we used poly-D-ornithine (P-ORN), poly-DLysine, (D-isomers are preferred for their resistance to proteases released by cells), and laminin. P-ORN and poly-D-Lysine are two positively-charged, synthetic polymers which enhance cell attachment to plastic and glass surfaces. They also increase adsorption of serum or extracellular matrix (ECM) proteins (such as laminin) to the culture substrate. Laminin, a major structural glycoprotein of the basement membrane, is a $90 \mathrm{kDa}$ glycoprotein composed of three polypeptide chains with a multidomain structure. It facilitates cell adhesion and growth by means of binding sites to cell membranes through integrin receptors and other plasma membrane-associated molecules (Haralson and Hassel, 1995).

For immunocytochemistry, coverslips ( $\varnothing 13 \mathrm{~mm}$, Menzel-Glaser, Braunschweig, Germany) were soaked in ethanol, flamed and placed in 24-wells plates. In order to ensure appropriate primary HC neuron culture conditions, plates were coated with P-ORN and laminin. Plates were first incubated for 4 to $6 \mathrm{~h}$ at RT with P-ORN $(1 \mathrm{mg} / \mathrm{ml})$ diluted 1:10 in sterile $\mathrm{H}_{2} \mathrm{O}_{\text {dist. }}$ (500 $\mu \mathrm{l} /$ well). After washing twice with $500 \mu \mathrm{l} /$ well sterile $\mathrm{H}_{2} \mathrm{O}_{\text {dist. }}$, plates were incubated with laminin 1:1000 in DMEM $(1 \mu \mathrm{g} / \mathrm{ml}, 500 \mu \mathrm{l} /$ well $)$ overnight at $37^{\circ} \mathrm{C}, 5 \% \mathrm{CO}_{2}, 95 \%$ humidity. Before transferring cells into wells, plates were washed twice with sterile DMEM (500 $\mu 1 /$ well). $500 \mu 1$ of the appropriate medium was added to each well and the plate kept at $37^{\circ} \mathrm{C}$.

For primary RGC culture, plates were coated for 30 min with poly-D-Lysine diluted 1:100 in sterile $\mathrm{H}_{2} \mathrm{O}_{\text {dist. }}$, washed twice with sterile $\mathrm{H}_{2} \mathrm{O}_{\text {dist }}$, incubated with laminin 1:1000 in DMEM and used for plating as described above for $\mathrm{HC}$ culture. 


\subsubsection{Primary hippocampal neuron culture}

To obtain primary $\mathrm{HC}$ neurons, hippocampi from rat Wistar embryos (embryonic day 18) were dissected and prepared as described previously (de Hoop, 1998).

Briefly, hippocampal tissues were collected in ice-cold CMF medium and centrifuged at 800 rpm $(68 \mathrm{~g})$ for $4 \mathrm{~min}$ at $4^{\circ} \mathrm{C}$. After removal of the medium, the cell pellet was incubated with $700 \mu \mathrm{l}$ trypsin $(0.25 \%) 15 \mathrm{~min}$ at $37^{\circ} \mathrm{C}$. Trypsin activity was then blocked by addition of 750 $\mu \mathrm{l}$ ice-cold FCS and $25 \mu \mathrm{l}$ DNase were added to dissolve DNA-aggregates released from damaged cells. The pellet was mechanically dissociated by gentle triturating with a firepolished Pasteur pipette (Brandt, Wertheim, Germany). After 3 min on ice to allow decanting of undissociated cells, the supernatant containing single cells was transferred into a new tube and centrifuged for $4 \mathrm{~min}$ at $800 \mathrm{rpm}(68 \mathrm{~g})$ at $4^{\circ} \mathrm{C}$. The pellet was then resuspended in the appropriate pre-warmed culture medium and cells were seeded in 24-well coated plates at a density of $2.5 \times 10^{5}$ cells/well containing $500 \mu \mathrm{l}$ of $\mathrm{HC}$ medium. Cultures were maintained at $37^{\circ} \mathrm{C}$ in $5 \% \mathrm{CO}_{2}$ and $95 \%$ humidity. $\mathrm{HC}$ cells remained in the same medium for the duration of the experiment (up to 15 days).

\subsubsection{Primary retinal ganglion cell culture}

Immunopurified rat RGC cultures were prepared and purified according to a two-step panning protocol, resulting in over 99.5\% purity, as described previously (Barres et al., 1988). Primary RGCs and macrophages both express the marker Thy-1. In order to specifically select RGCs from a retinal cell homogenate, cells are sequentially incubated on plates coated with different antibodies. Macrophages are first removed from the cell suspension by incubation on plates coated with anti-macrophages antibodies. RGCs are then selected for their affinity on a plate coated with Thy-1 antibodies.

Briefly, macrophage-panning plates were coated overnight at $4^{\circ} \mathrm{C}$ with goat anti-rabbit IgG (1:400, specific for rabbit anti-macrophage antibodies) in Tris- $\mathrm{HCl} 50 \mathrm{mM} \mathrm{pH} \mathrm{9.5,} \mathrm{while}$ RGC-panning plates were coated with goat anti-mouse $\operatorname{IgM}(1: 600$, specific for mouse antiThyl-antibodies) in Tris- $\mathrm{HCl} 50 \mathrm{mM} \mathrm{pH} \mathrm{9.5.} \mathrm{After} \mathrm{washing,} \mathrm{macrophage-panning} \mathrm{plates}$ were blocked with BSA $0.2 \%$ in DPBS, while RGC-panning plates were incubated at RT with mouse anti-Thy- 1 antibody in DPBS $0.2 \%$ BSA. Wistar rat pups were sacrificed on postnatal day 7-8 by decapitation and the eyes extracted. The cornea, the lens and the vitreous body 
were removed, and the retinas collected in EBSS. After removal of the medium, tissues were incubated with papain (165 units) $15 \mathrm{~min}$ at $37^{\circ} \mathrm{C}$. Papain activity was then blocked by addition of $750 \mu 1 \mathrm{x}$ Low Ovomucoid and $100 \mu \mathrm{l}$ DNase and macrophages were labelled by the anti-macrophages antibodies present in the solution. Retinas were centrifuged $11 \mathrm{~min}$ at $1000 \mathrm{rpm}(106 \mathrm{~g})$ at $25^{\circ} \mathrm{C}$ and resuspended in 1x High Ovomucoid solution. After a second 11 min centrifugation at $1000 \mathrm{rpm}(106 \mathrm{~g})$ at $25^{\circ} \mathrm{C}$, tissues were dissociated and filtered through a sterile Nylon mesh (Heidland, Gütersloh, Germany) using DPBS 0.02\% BSA to avoid cell aggregates. Blocking solution was removed from macrophage-panning plates coated with anti-rabbit-IgG. The cell suspension was incubated on them twice subsequently ( $20 \mathrm{~min}$ and $30 \mathrm{~min}$ at RT) to bind to and remove macrophages present in solution. Cell suspension was then incubated 45 min at RT on RGC-panning plates coated with goat anti-mouse and mouse anti-Thy-1 antibodies to specifically select RGCs. After minutious washing with DPBS to ensure high culture purity, RGCs were detached from the plate by addition of $200 \mu 1$ Trypsin $2.5 \%\left(15 \mathrm{~min}\right.$ at $\left.37^{\circ} \mathrm{C}\right)$ and centrifuged $18 \mathrm{~min}$ at $800 \mathrm{rpm}(68 \mathrm{~g})$ at $25^{\circ} \mathrm{C}$.

For immunocytochemistry, RGCs were plated at a density of 7000 cells/well on 24 wellplates containing coverslips coated with poly-D-lysine and containing $500 \mu$ l Neurobasal SATO medium. One day after plating, half of the medium was removed and $1.5 \times 10^{8}$ i.u. of viral vectors were applied in the remaining $250 \mu \mathrm{l}$ of medium. Medium was refilled to $500 \mu 1$ the following day. For Raf-1 kinase inhibition studies, $500 \mathrm{nM}$ of Raf-1 kinase inhibitor GW5074 or DMSO were added to the medium one day after virus application and kept for the experiment duration (incubation time 3 days). Inhibitor and DMSO application was renewed after two days.

\subsubsection{CSM culture}

CSM14.1 is a rat nigrostriatal cell line immortalized by introduction of the temperaturesensitive simian virus 40 large $\mathrm{T}$ antigen and a Bcl-2 transfectant CSM14.1-Bcl-2 (Zhong et al., 1993). For this study, wild-type CSM14.1 cells and CSM14.1 cells stably transfected with a mouse Flag-BAG1 (Kermer et al., 2002) were used.

Wild-type cells and BAG1 stably transfected cells were maintained in DMEM supplemented with $10 \%$ FCS, $1 \mathrm{mM}$ L-glutamine, 100 units of penicillin/ml, and $100 \mu \mathrm{g}$ of streptomycin sulphate $/ \mathrm{ml}$, at either $32^{\circ} \mathrm{C}$ (permissive temperature) or $39^{\circ} \mathrm{C}$ (non-permissive temperature) 
for one week (Zeiner et al., 1997). Pyromycin was added to the BAG1 cell medium for selection of the stably transfected cells.

CSM wild-type and BAG1 overexpressing cells exhibit different proliferation rates. For lysates, CSM wild-type and BAG1 cells were therefore plated at a density of $2.3 \times 10^{5}$ and 7.4 $\mathrm{x} 10^{5}$ per Petri dish respectively to achieve equal densities at switch to non-permissive temperature. For immunofluorescence analysis, wild-type and stably transfected CSM cells were seeded on coverslips $(\varnothing 10 \mathrm{~mm})$ in 24 -well plates at a density of $3.8 \times 10^{4}$ cells/well. Cultures were maintained at $32^{\circ} \mathrm{C}$ or $39^{\circ} \mathrm{C}$ in $5 \% \mathrm{CO}_{2}$ and $95 \%$ humidity. One day before fixation, cells were trypsinized and re-plated at a density of 1:3 and 1:1 respectively to allow single cell visualisation.

For Raf-1 kinase inhibition studies, cells were treated for $24 \mathrm{~h}$ before lysis with 500, 1000 or 2000 nM Raf-1 kinase inhibitor GW5074 or DMSO, respectively.

\subsubsection{Immunocytochemistry}

Immunocytochemistry was used to investigate expression or localisation of proteins of interest within the cells. Cells grown on coverslips were first fixed in PFA 4\% $10 \mathrm{~min}$ at RT. After 2 x PBS washes and transfer on parafilm support (Brandt, Wertheim, Germany), cells were permeabilised by application of $100 \%$ ice-cold acetone $10 \mathrm{~min}$ at $-20^{\circ} \mathrm{C}$, washed twice with PBS and blocked with 10\% new-born goat serum in PBS 10 min at RT to avoid any unspecific binding. Primary antibodies were applied at respective dilutions (Table 5) at $4{ }^{\circ} \mathrm{C}$ overnight. Following 2 x PBS washes, appropriate fluorescently-labelled secondary

\begin{tabular}{llc}
\hline Antigen & Species & Working dilution \\
\hline Cy2-anti-Mouse IgG & Mouse & $1: 250$ \\
Cy2-anti-Rabbit IgG & Rabbit & $1: 250$ \\
Cy3-anti-Mouse IgG & Mouse & $1: 250$ \\
Cy3-anti-Rabbit IgG & Rabbit & $1: 250$ \\
Cy5 anti-Goat IgG & Donkey & $1: 50$ \\
Cy5 anti-Mouse IgG & Mouse & $1: 100$ \\
Cy5-anti-Rabbit IgG & Goat & $1: 50$ \\
Phospho-ADF-cofilin & Rabbit & $1: 500$ \\
Raf-1 (E-10) & Mouse & $1: 50$ \\
Rhodamine-conjugated phalloidin & & $1: 500$ \\
ROCK2 (C-20) & Goat & $1: 50$ \\
\hline
\end{tabular}

Table 5: $\quad$ Respective working dilutions of the antibodies and fluorescently-labelled molecules used for immunocytochemistry. 
antibodies were then applied for 45 min at $37^{\circ} \mathrm{C}$. After $2 \times$ PBS washes, cells were nuclear counterstained with 4', 6-diamidino-2-phenylindole (DAPI) and mounted in mowiol. Fluorescence of samples was observed and recorded on a Zeiss Axioplan 2 microscope equipped with a CCD camera and AxioVision 3 software (Zeiss), a Zeiss Apotome ${ }^{\mathrm{TM}}$ device and AxioVision Rel. 4.6 software (Zeiss), or a Leica SP2 confocal laser scanning microscope equipped with an acoustooptical beam splitter.

\subsubsection{Protein handling procedures}

\subsubsection{Protein lysates}

For protein lysate preparation, culture medium was removed from the plates and cells were washed twice in PBS. Cells were collected in $100 \mu \mathrm{l}$ TBS lysis buffer using a cell scraper (Petri dish) or a pipette tip (24-well plates) and put on ice for $15 \mathrm{~min}$. Samples were sonicated 30 seconds at $45 \%$ power and centrifuged $15 \mathrm{~min}$ at maximal speed at $4{ }^{\circ} \mathrm{C}$ in a microcentrifuge. The supernatant was then transferred into a new tube and stored at $-20^{\circ} \mathrm{C}$.

\subsubsection{Protein concentration determination}

Protein concentration was determined by Lowry assay. In basic conditions, proteins have the ability to reduce $\mathrm{Cu}^{2+}$ into $\mathrm{Cu}^{+}$. These ions form complexes with 2 molecules of bicinchoninic acid (BCA) and produce a violet compound (absorbance at $562 \mathrm{~nm}$ ). Absorbance is proportional to the amount of complexes created, therefore to protein amount.

Solutions A and B of BCA were mixed 1:50 and $200 \mu \mathrm{l}$ of the obtained solution were added to either 5 or $10 \mu \mathrm{l}$ of protein samples to quantify in a 96 -well plate. All samples were tested in duplicate. Simultaneously, a protein standard curve was prepared with BSA in a concentration range between 0.6 and $40 \mathrm{mg} / \mathrm{ml}$. The plate was then incubated $30 \mathrm{~min}$ at $60^{\circ} \mathrm{C}$ and absorbance was measured at $562 \mathrm{~nm}$ on a TECAN Rainbow plate reader using the easyWINbasic software. Protein concentration was determined by correlation between sample absorbance and the standard curve slope.

\subsubsection{Sodium dodecyl sulphate-polyacrylamide gel electrophoresis (SDS-PAGE)}

SDS-PAGE is a method allowing the separation of a large range of proteins of various molecular weights and charges. It is based on the following principle: proteins are denatured by heat and a reducing agent (SDS). Proteins are concentrated into the stacking gel and are separated within the resolving gel upon their molecular weight, the biggest ones being slowed 
down most by the gel matrix. This matrix is constituted of polyacrylamide/bis-acrylamide (Rotiphorese), the cross-linking of which is catalysed by free radicals produced by addition of ammonium peroxide and TEMED.

For equal loading onto the gel, protein concentrations of samples were adjusted in lysis buffer to reach a final volume of $12 \mu \mathrm{l}$ or $20 \mu \mathrm{l}$ (according to lane volumes) and mixed with SDS protein loading buffer $+10 \%$ of $5 x \beta$-mercaptoethanol (final concentration $1 \mathrm{x}$ ). Samples were then heated $5 \mathrm{~min}$ at $95^{\circ} \mathrm{C}$, briefly centrifuged at RT and equal amounts of protein were loaded into the gel lanes. If not otherwise specified, $4 \%$ stacking gels and $10 \%$ resolving gels were used. SDS-PAGE was run at $4^{\circ} \mathrm{C}$ at 75 Volts for $15 \mathrm{~min}$ to allow samples to enter the gel and to be collected in the stacking gel. Then proteins were separated in the resolving gel by application of a 120 Volts electric field for approximately $90 \mathrm{~min}$, depending on the size of the proteins of interest. The molecular weight of loaded proteins was defined by comparison with a molecular weight marker (Dual marker) loaded and separated in parallel.

\subsubsection{Western blotting}

Western blotting (Towbin et al., 1979; Burnette, 1981) is a technique allowing the detection and quantification of a protein of interest previously subjected to SDS-PAGE by a specific primary antibody. Briefly, protein samples are transferred from a SDS-resolving gel to a nitrocellulose membrane by application of an electric current. The membrane is subsequently incubated with unlabeled primary antibody specific for the target protein. The antibody is then detected by addition of secondary immunological reagents (e.g. anti-immunoglobulin) coupled to Horseradish peroxydase (HRP) and their reactive substrates (chemiluminescence).

Proteins, previously treated with $5 \mathrm{x}$ SDS protein loading buffer, heated at $95^{\circ} \mathrm{C}$ for $5 \mathrm{~min}$ and loaded on SDS-PAGE gel (section 2.3.4.3), were transferred onto a nitrocellulose membrane (AppliChem, Darmstadt, Germany) during $90 \mathrm{~min}$ at 100 Volts. The membrane was then blocked with 5\% milk in Tris-buffered saline/Tween-20 (TBS-T) for $1 \mathrm{~h}$ at RT to avoid unspecific binding of the antibody, prior to incubation with primary antibodies overnight at $4^{\circ} \mathrm{C}$. Respective dilutions of the antibodies used in this study are listed in the Table 6 . After 3 $\mathrm{x} 15$ min washing in TBS-T, membranes were incubated with the corresponding HRPcoupled secondary antibodies (1:1000) for $1 \mathrm{~h}$ at RT. Following 3 x 15 min washing in TBS-T 


\begin{tabular}{llc}
\hline Antigen & Source & Working dilution \\
\hline BAG1 & Rabbit & $1: 1000$ \\
Flag-M2 & Mouse & $1: 2000$ \\
Gapdh & Mouse & $1: 10000$ \\
p44/42 MAPK & Rabbit & $1: 1000$ \\
Phospho-c-Raf & Rabbit & $1: 500^{*}$ \\
Phospho-p44/42 MAPK (Thr202/Tyr204) & Rabbit & $1: 1000^{*}$ \\
Phospho-SAPK/JNK & Mouse & $1: 100$ \\
Raf-1 (E-10) & Mouse & $1: 350$ \\
RhoA & Mouse & $1: 350$ \\
ROCK2 (C-20) & Goat & $1: 500$ \\
\hline
\end{tabular}

Table 6: Dilutions of antibodies used for western blotting. Antibodies were either diluted in milk 5\% or BSA $5 \%(*)$ according to the manufacturer's instructions.

equal volumes of ECL solutions 1 and 2 were applied for $1 \mathrm{~min}$ in the dark on the membrane. Chemiluminescence was visualized and quantified via a Fluor- $\mathrm{S}^{\mathrm{TM}}$-max imager and Quantity One software (version 4.2.1).

\subsubsection{Subcellular fractions}

The ProteoExtract ${ }^{\circledR}$ Subcellular Proteome Extraction Kit allows the differential extraction of proteins from culture cells according to their subcellular localization. The sequential extraction steps yield four fractions containing cytosolic proteins, plasma membrane and organelle proteins, soluble nuclear proteins, and cytoskeletal and nuclear matrix proteins. All extraction steps were performed in presence of Protease Inhibitor cocktail and the endonuclease Benzonase ${ }^{\circledR}$ Nuclease according to the manufacturer's instructions.

\subsubsection{ROCK activity assay}

The CycLex ROCK Kinase Activity Assay is based on the ELISA principle. Wells are coated with a myosin-binding subunit of myosin phosphatase (MBS), a substrate phosphorylated by both ROCK isoforms. Addition of the sample causes substrate phosphorylation. Following sample removal and plate washing, a HRP-conjugated antibody recognising the phosphorylated form of MBS is added. Further addition of HRP substrate induces a colorimetric reaction proportional to ROCK activity in the sample.

ROCK activity assay was performed according to the manufacturer's instructions using 0.25 $\mu \mathrm{g}$ protein. Briefly, $100 \mu \mathrm{l}$ of the sample was added to the wells and incubated $30 \mathrm{~min}$ at $30^{\circ} \mathrm{C}$. Wells were then washed, $100 \mu \mathrm{l}$ of HRP-conjugated anti-phospho specific antibody added to each well and the plate incubated for $1 \mathrm{~h}$ at RT. Following washes, $100 \mu \mathrm{l}$ of 
substrate reagent were added to each well and left 15 min at RT. Reaction was then stopped by addition of $100 \mu \mathrm{l}$ of Stop solution and the absorbance was measured at $450 \mathrm{~nm}$ on a TECAN Rainbow plate reader using the easyWINbasic software.

\subsubsection{Animal procedures}

\subsubsection{In vivo experiments}

All animal experiments were carried out according to the regulations of the local animal research council and legislation of the State of Lower Saxony (Braunschweig). Wistar rats, purchased from Charles River (Sulzfeld, Germany) and transgenic BAG1 ${ }^{+/}$mice and wildtype littermate (FVB/n, Taconic Inc., Germantown, NY, USA, Kermer et al., 2002), were housed at a $12 \mathrm{~h} / 12 \mathrm{~h}$ of light/dark cycle, provided with food and water ad libitum. All animals were sacrificed by $\mathrm{CO}_{2}$ inhalation.

For axotomy and optic nerve crush, adult female Wistar rats (200-300 g) were used. The numbers of animals used for each treatment group and experiment are listed in Table 7. Axotomy groups of transgenic $\mathrm{BAG}^{+/-}$mice and wild-type littermate consisted of the following animal numbers: wild-type: $n=3, \mathrm{BAG}^{+/-}: \mathrm{n}=4$.

\begin{tabular}{lccc}
\hline Experiment & \multicolumn{3}{c}{ Treatment group } \\
\hline & PBS & AAV.EGFP & AAV.BAG1 \\
\hline Axotomy d0 & 4 & 4 & 3 \\
Axotomy d-21 & 4 & 5 & 6 \\
Optic nerve crush & 3 & 4 & 4 \\
\hline
\end{tabular}

Table 7: $\quad$ Animal groups used for axotomy and optic nerve crush.

\subsubsection{Injection of $A A V-2$ vectors}

Injections were performed either upon brief anaesthesia by diethyl ether inhalation 3 weeks before operation, or under general anaesthesia (chloral hydrate, $420 \mathrm{mg} / \mathrm{kg}$ body weight, intraperitoneally) at the time of the surgery. Each injection consisted of 2 or $3 \mu$ containing $1.5 \times 10^{9} \mathrm{AAV}$ genomes (equivalent to $5 \times 10^{7}$ i.u.) or an equivalent volume of PBS. Using an operating microscope, the solution was injected into the intravitreal space, puncturing the eyeball below the cornea-sclera junction by means of a glass microelectrode (tip diameter 30 $\mu \mathrm{m})$ coupled to a Hamilton syringe. Care was taken to avoid any penetration of the lens. 


\subsubsection{Anaesthesia and surgical approach}

The anaesthesia was performed by intraperitoneal injection of chloral hydrate $(420 \mathrm{mg} / \mathrm{kg}$ body weight). A similar surgical approach was used in both experimental paradigms to access the optic nerve. The skin was incised close to the superior orbital rim and the orbital were opened taking care not to damage the supraorbital vein. The intraorbital glands were partially sectioned and the superior extraocular muscles were spread by means of a small retractor. The optical nerve was exposed by longitudinal incision of the eye retractor muscle and the optic nerve sheath.

After all surgical procedures retinal blood supply was verified by fundoscopy and animals with persistent retinal ischemia were excluded.

\subsubsection{Optic nerve axotomy}

Upon transection of the optic nerve, RGCs undergo a well-characterised apoptotic cell death, with a degeneration of $85-90 \%$ of the deafferented cells 14 days after the lesion (Bähr, 2000; Cellerino et al., 2000b). This time point has previously been proven to be appropriate to determine whether neuroprotective strategies are successful in this model (Kermer et al., 1998).

Axotomy of the optic nerve was performed essentially as described previously (Kermer et al., 2001; Lingor et al., 2005). The optic nerve was transected at a distance of ca. $2 \mathrm{~mm}$ from the posterior pole of the eye without damaging the retinal blood supply. In order to retrogradely label RGCs, a 2x2 mm piece of gel foam (Braun, Melsungen, Germany) was soaked in Fluorogold (Hydroxystilbamidine; Bio-Trend, Cologne, Germany) and placed on the optic nerve stump. On day 14 post-axotomy, animals were sacrificed by $\mathrm{CO}_{2}$ inhalation, the eyes were extracted and processed for quantification of RGC survival as described in section 2.3.6.1.

\subsubsection{Optic nerve crush}

The crush of the optic nerve is a model where the integrity of nerve is partially conserved. Lesioned axons remain in place, but face a non-permissive environment for regeneration (Sabel and Aschoff, 1993). 
For optic nerve crush experiments, ligation of the optic nerve was performed using a 10/0 surgical suture (Ethicon, Johnson-Johnson, Livingston, United Kingdom) for $30 \mathrm{sec}$, resulting in a complete transection of all RGC axons. The suture was removed and the operative access closed. Rats were sacrificed on day 28 after surgery by $\mathrm{CO}_{2}$ inhalation and immediately perfused by transcardial injection of $250 \mathrm{ml}$ PBS and $200 \mathrm{ml} \mathrm{4 \%} \mathrm{PFA} \mathrm{(see} \mathrm{section} \mathrm{2.3.5.6).}$ The eye and the optic nerve were removed and processed for immunohistochemistry and quantification of RGC regenerating fibres as described in sections 2.3.5.7 and 2.3.6.2.

\subsubsection{Transcardial perfusion}

Transcardial perfusion is a method commonly used for tissue fixation in immunohistochemical protocols. Delivery of the fixative solution is based on the animal's circulatory system, which allows for an even distribution throughout the body tissues. Fixation ensures preservation of the tissue ultrastructure and stabilisation of protein and peptide conformation, so that antibodies can bind to antigen sites.

For preparation of optic nerve sections, animals were deeply anesthetised by $\mathrm{CO}_{2}$ inhalation and immediately intracardiacally perfused with PBS followed by 7\% PFA. For this, animals were fixed on a rack, the abdominal cavity opened, and the diaphragm cut to facilitate the access to the thorax. Using large scissors, the thoracic cage was bilaterally cut and opened to allow access to the heart. Following incision of the left ventricle, a catheter connected to a blunt-ended needle was inserted through the ventricle and atrium into the aorta. Needle position was secured in position by clamping and perfusion was restricted to the upper part of the body by clamping the abdominal aorta. The liver and nose were cut to allow a large part of the blood volume to leave the body and to control the perfusion of the neurocranium, respectively. The position of the needle was considered as appropriate when PBS flow was detected from the cut nose. After clearance of blood from the body (by using ca. $200 \mathrm{ml}$ PBS), PBS was replaced with ice-cold 7\% PFA (200 ml). Eyeballs and adjacent optic nerves were then carefully extracted, and the cornea, the lens and the vitreous body were removed. The remaining eye cup, containing the retina and the adjacent optic nerve, were postfixed in $4 \%$ PFA in PBS for $1 \mathrm{~h}$. For cryoprotection, tissues were then dehydrated in $30 \%$ sucrose at $4{ }^{\circ} \mathrm{C}$ for $24 \mathrm{~h}$ and kept at $-20^{\circ} \mathrm{C}$ for further processing. 


\subsubsection{Immunohistochemistry}

After embedding the previously fixed tissues in Cryomatrix, longitudinal sections $(16 \mu \mathrm{m}$ thickness) of the optic nerve were prepared using a Leica cryostat, collected on gelatinecoated glass slides and stored frozen.

For immunohistochemistry, specimens were dried at $37^{\circ} \mathrm{C}$ for $1 \mathrm{~h}$ and antigen retrieval was performed for $4 \mathrm{~h}$ in TBS-T (pH 9.0) at $60^{\circ} \mathrm{C}$. Unspecific binding was blocked by application of 10\% new-born goat serum, and anti-GAP43 antibody was applied in a 1:250 dilution at $4^{\circ} \mathrm{C}$ overnight in order to stain regenerating fibres. After 2 x PBS washes, secondary Cy3labelled anti-rabbit antibody (1:250) was applied for $45 \mathrm{~min}$ at RT. The sections were then nuclear counter-stained with DAPI and embedded in mowiol.

\subsubsection{Data analysis}

\subsubsection{Quantification of surviving RGCs}

On day 14 after axotomy, animals were sacrificed by lethal $\mathrm{CO}_{2}$ inhalation and the eyes were extracted. The cornea, the lens and the vitreous body were removed, and the remaining eye cup containing the retina was fixed in 4\% PFA in PBS, pH 7.4 for $1 \mathrm{~h}$. Retinas were then extracted and flat-mounted on object-slides in glycerol-PBS (1:1).

The vector-transduced area of the retina was identified by visualization of EGFP-expressing cells. In each retinal quadrant of the transduced and non-transduced areas, 3 fields of 62,500 $\mu \mathrm{m}^{2}$ were counted at eccentricities of one-sixth, one-half and five-sixths of the retinal radius. The number of Fluorogold-positive RGCs was determined by fluorescence microscopy (Zeiss-Axioplan) using a commercial Fluorogold filter $(365 / 420 \mathrm{~nm})$. EGFP fluorescence from strongly labelled amacrine neurons is reported to show some leak-through into this filter at longer exposure times (Malik et al., 2005). Therefore, only cells displaying a round shape and punctuate perinuclear Fluorogold staining were counted as RGCs. RGC counts were performed independently by two different investigators according to a blinded protocol.

\subsubsection{Quantification of regenerating RGC axons}

For evaluation of RGC axon regeneration following optic nerve crush, slides were immunostained for GAP43 (see section 2.3.5.7) and photomicrographs were taken using a fluorescence microscope (Zeiss-Axioplan) equipped with a CCD camera and AxioVision 3 software (Zeiss). Images of whole sections were assembled from single pictures taken with a 
20x objective. The number of regenerating neurons at designated distances from the crush was evaluated using a counting grid superimposed on to the photomicrograph.

\subsubsection{Quantification of neurite length in primary RGCs}

Primary RGCs expressing sufficient EGFP reporter gene (day 5 after virus application) were incubated in a microscope climate chamber for live cell imaging $\left(37^{\circ} \mathrm{C}, 5 \% \mathrm{CO}_{2}\right)$ on a fluorescence inverted microscope (Zeiss-Axiovert) equipped with CCD camera and AxioVision Rel. 4.6 software (Zeiss). Contrast phase photographs of at least two random visual fields per culture well were taken with a 10x objective and neurite length was evaluated semi-automatically using the axon tracing module of "Image J" (Free Java software provided by the National Institutes of Health, Bethesda, Maryland, USA). Total neurite length was divided by cell number and expressed as averaged neurite length.

\subsubsection{Quantification growth cone surface and lamellipodia in primary RGCs}

For evaluation of RGC growth cone surface, photomicrographs of cells stained with antiphospho-ADF-cofilin antibodies (for immunohistochemistry, see section 2.3.5.7) were taken with a $63 \mathrm{x}$ oil immersion objective (Zeiss) using a fluorescence microscope (Zeiss-Axiovert) equipped with a Zeiss Apotome ${ }^{\mathrm{TM}}$ device, a CCD camera and AxioVision Rel. 4.6 software (Zeiss). The Apotome ${ }^{\mathrm{TM}}$ device allows the recording of "pseudo-confocal" images by subtracting the fluorescence of nearby layers and further processing of images by the software. Pictures of single cells were taken randomly on different samples. Growth cone surface was measured and quantified using "Image J" (Free Java software provided by the National Institutes of Health, Bethesda, Maryland, USA). Streak-like structures protruding from the growth cone area were counted as lamellipodia.

\subsubsection{Statistics}

Experiments were performed at least in triplicate. $\mathrm{n}$ are indicated in the appropriate method sections if not mentioned in the text. Differences between groups were considered statistically significant according to a one-way ANOVA followed by a parametric multiple comparison test (Dunnett test). Significances are indicated with $* \mathrm{p}<0.05,{ }^{* *} \mathrm{p}<0.01,{ }^{* * *} \mathrm{p}<0.001$, unless otherwise stated. 


\section{Results}

\subsection{Overexpression of BAG1 by bicistronic AAV-2 vectors}

\subsubsection{Cloning of the AAV-2 expression cassette}

In order to investigate the effects of BAG1 in different in vivo models of apoptosis and regeneration, the cDNA of mouse Flag-BAG1 was first cloned into a bicistronic recombinant adeno-associated virus (AAV) vector. The AAV-2 serotype was chosen because of its ability to specifically transfect neurons, i.e. RGCs in our paradigm (Tenenbaum et al., 2004). The vector expressed BAG1 (tagged with the Flag epitope) under control of the human synapsin 1 gene (hSyn1) promoter and, in a separate expression cassette, EGFP also driven from the hSyn1 promoter (AAV.BAG1), as depicted in Figure 11. As a negative control, we used an AAV-2 vector which expresses EGFP from a hSyn1 promoter and containing a disabled Bcl$\mathrm{X}_{\mathrm{L}}$ expression cassette (AAV.EGFP, Figure 11, Malik et al., 2005).

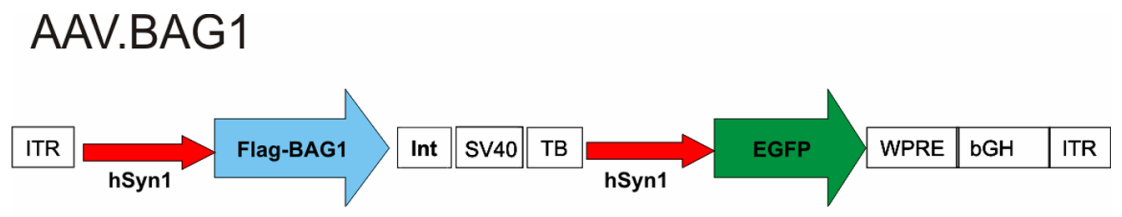

\section{AAV.EGFP}

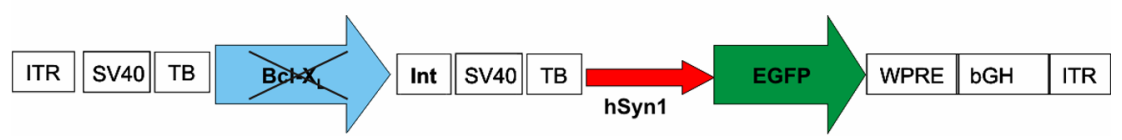

Figure 11: Schematic depiction of the AAV-2 vector genomes. ITR, AAV-2 inverted terminal repeats; hSyn1, human synapsin 1 gene promoter; Flag-BAG1, mouse BAG1 cDNA N-terminally tagged with the Flag epitope; Bcl- $\mathrm{X}_{\mathrm{L}}$, rat Bcl- $\mathrm{X}_{\mathrm{L}}$ cDNA N-terminally tagged with the Flag epitope (disabled, not transcribed); Int, intron; SV40, simian virus 40 polyadenylation site; TB, synthetic transcription blocker; WPRE, woodchuck hepatitis virus posttranscriptional regulatory element; $\mathrm{bGH}$, bovine growth hormone polyadenylation site.

\subsubsection{Analysis of transgene expression}

We confirmed transgene expression levels from the vector in a primary hippocampal (HC) neuron culture. Figure 12 shows that application of $1 \times 10^{8}, 5 \times 10^{7}$ or $1 \times 10^{7}$ i.u. of AAV.BAG1 induced the expression of the EGFP reporter gene in a concentration-dependent manner, while no expression was observed in non-treated controls. 

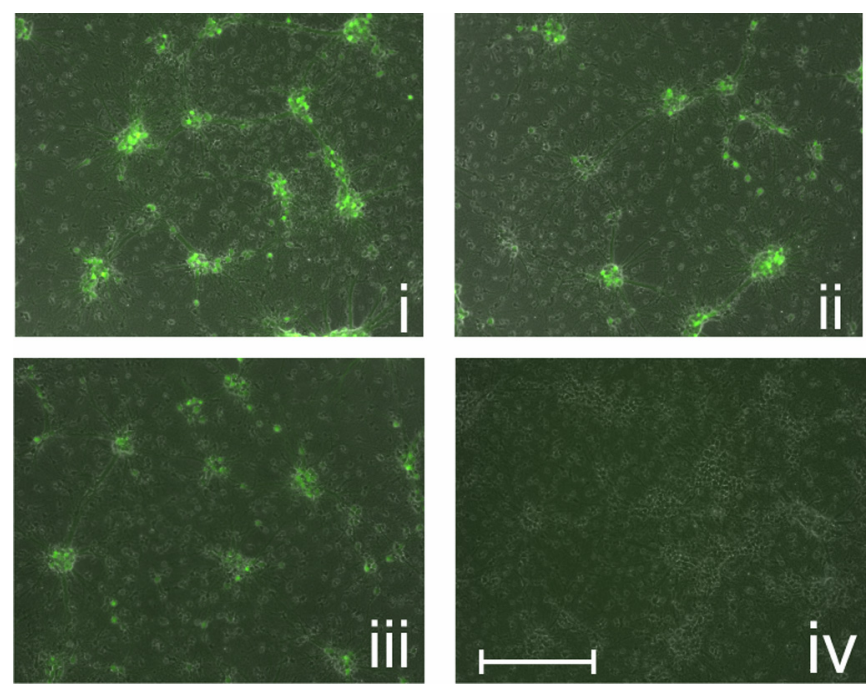

Figure 12: $\quad$ Titration of AAV.BAG1 in vitro. Application of $1 \times 10^{8}$ (i), 5 x $10^{7}$ (ii) and 1 x $10^{7}$ (iii) i.u. AAV.BAG1 respectively induces the expression of EGFP reporter gene in a concentrationdependent manner in primary HC neurons. Non-treated control (iv). Scale bar: $200 \mu \mathrm{m}$.

Expression of Flag-BAG1 was then confirmed by western blot of primary HC neuron lysates. Application of $1 \times 10^{8}$ and $5 \times 10^{7}$ i.u. of AAV.BAG1 resulted in the expression of FlagBAG1 in a concentration-dependent manner. Expression of endogenous BAG1 (Figure 13, upper panel, lower band) is visible in both control and infected cultures at similar levels. Expression of the Flag-BAG1 construct was additionally verified by anti-Flag-M2 antibody and showed that only AAV.BAG1-treated cells expressed the Flag-BAG1 transgene. The titre of $5 \times 10^{7}$ i.u. showed sufficient transduction and transgene expression, and was thus further used in in vivo experiments.

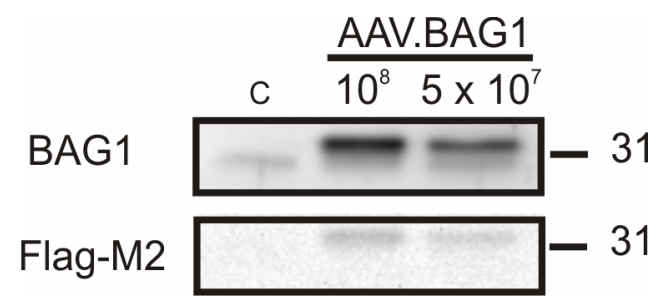

Figure 13: Analysis of transgene expression by Western blot. Application of 1 x $10^{8}$ and $5 \times 10^{7}$ i.u. AAV.BAG1 on primary HC neurons induces the expression of BAG1 in a concentrationdependent manner. Endogenous expression of BAG1 (lower band), visible in control and infected cultures, was used as loading control. Expression of the Flag-BAG1 construct was additionally verified by anti-Flag-M2 antibody. Size standards in kDa are shown on the right. 


\section{2 In vivo studies}

\subsubsection{Experimental setup}

The effects of BAG1 on apoptosis and regeneration were then tested in two different in vivo models, the optic nerve axotomy (model for apoptosis) and optic nerve crush (model for regeneration). The experimental setup is schematically depicted in Figure 14.

\section{Axotomy}

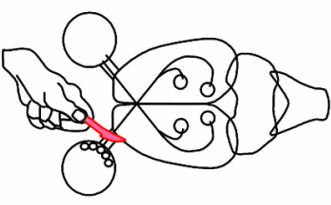

Crush

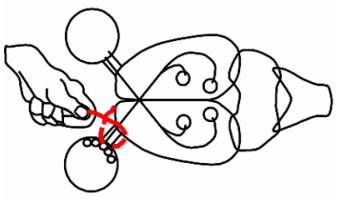

i.o. injection

of virus

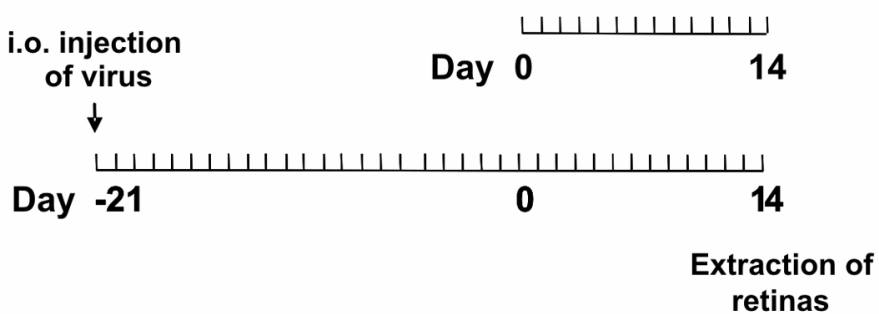

i.o. injection of virus $\downarrow$

Day 0

Extraction of optic nerves

Figure 14: Experimental setup of in vivo studies. Intravitreal (i.o.) injections of AAV.BAG1 and AAV.EGFP were performed 3 weeks prior or directly after operation (arrow). Retinas and optic nerves were taken 14 or 28 days following axotomy or crush respectively, and processed for RGC counting or immunohistochemistry.

For axotomy, the virus was applied either 21 days prior to surgery (day -21) to allow for proper protein expression before lesion, or at the time of axotomy (day 0) to mimic a possible therapeutic application. Animals were sacrificed 14 days after axotomy for evaluation of RGC survival. The optic nerve crush model was used to assess the regeneration response of RGC axons in a non-permissive CNS environment. The virus was applied at the time of surgery (day 0). Animals were sacrificed on day 28 after surgery for quantification of axonal regeneration.

\subsubsection{RGC survival after axotomy-induced apoptosis is increased by BAG1}

It has been previously shown that BAG1 plays an important role in anti-apoptosis in vitro (Takayama et al., 1995; Bardelli et al., 1996; Clevenger et al., 1997). The optic nerve axotomy is an established model for the in vivo evaluation of neuroprotective effects in lesion-induced apoptosis. In order to investigate whether BAG1 protects RGCs from 
axotomy-induced apoptosis, we compared the effects of intravitreal application of AAV.BAG1 and AAV.EGFP. RGCs were retrogradely labelled by administration of Fluorogold after the axotomy procedure and surviving RGCs were counted at day 14 postaxotomy in retinal flatmounts. Intravitreal application of $5 \times 10^{7}$ i.u. of AAV.BAG1 or AAV.EGFP resulted in an infection of approximately $1 / 3$ of the retina around the injection site (Figure 15).

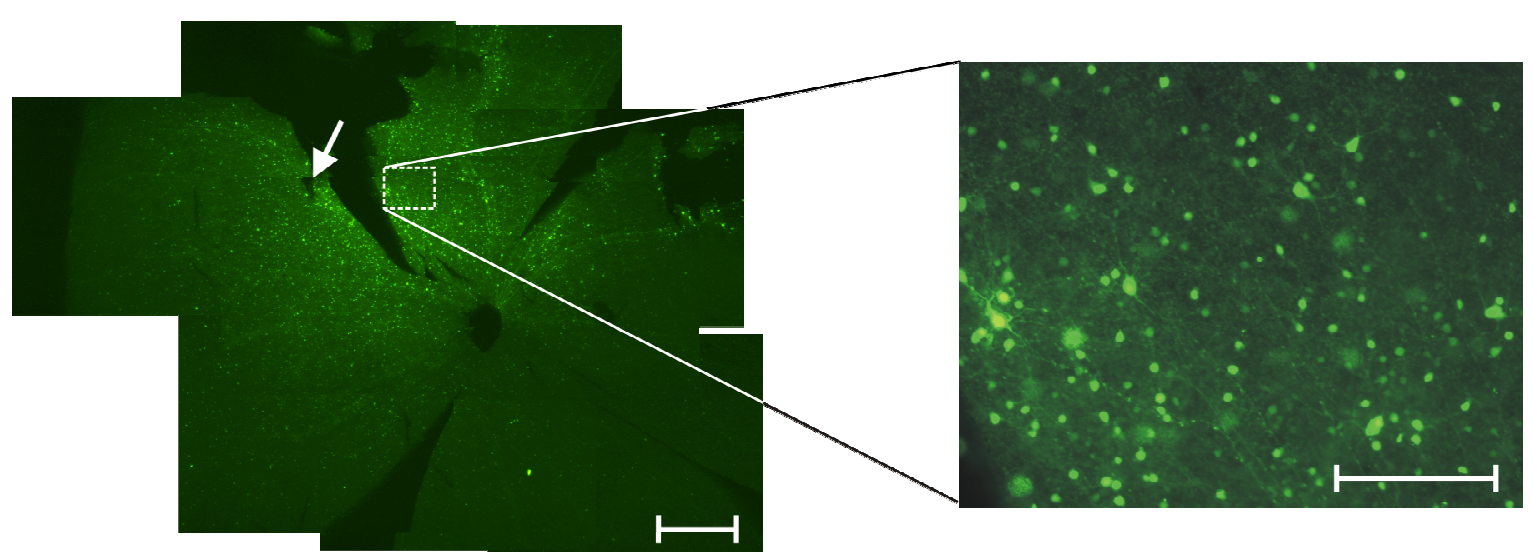

Figure 15: $\quad$ EGFP expression in RGCs (retinal flatmount) after intravitreal injection of $5 \times 10^{7}$ i.u. of AAV.BAG1 following axotomy. Approximately $1 / 3$ of the retina is transduced around the injection site (arrow). Scale bar: $200 \mu \mathrm{m}$. Scale bar in insert: $100 \mu \mathrm{m}$.

The effect of BAG1 on RGC survival was evaluated by comparison of RGC survival in transfected and non-transfected areas of the same retinas. Non-transfected and AAV.EGFP transfected areas did not significantly differ from PBS-treated controls (Figure 16.A). When AAV.BAG1 was injected at the day of axotomy, only a trend to increased RGC survival was observed, which is most likely due to insufficient transgene expression by the AAV vector. In contrast, virus application three weeks prior to axotomy allowed for sufficient expression of BAG1 and this resulted in a significant increase in surviving RGCs $\left(1572 \pm 84 \mathrm{RGC} / \mathrm{mm}^{2}\right)$ in comparison to PBS and AAV.EGFP-treated animals $\left(531 \pm 66\right.$ and $780 \pm 102 \mathrm{RGC} / \mathrm{mm}^{2}$, Figure 16.A). This correlates with results obtained from optic nerve axotomies in transgenic mice overexpressing BAG1 (Figure 16.B). At 14 days post-axotomy, BAG1 $1^{+/-}$mice showed a $\sim 50 \%$ increase in RGC survival $\left(2867 \pm 22,2788 \pm 87\right.$ and $2858 \pm 124 \mathrm{RGC} / \mathrm{mm}^{2}$ in the inner, middle and outer retinal radiuses, respectively) compared to their wild-type littermates $\left(1805 \pm 46,2024 \pm 63\right.$ and $2025 \pm 51 \mathrm{RGC} / \mathrm{mm}^{2}$, respectively). 

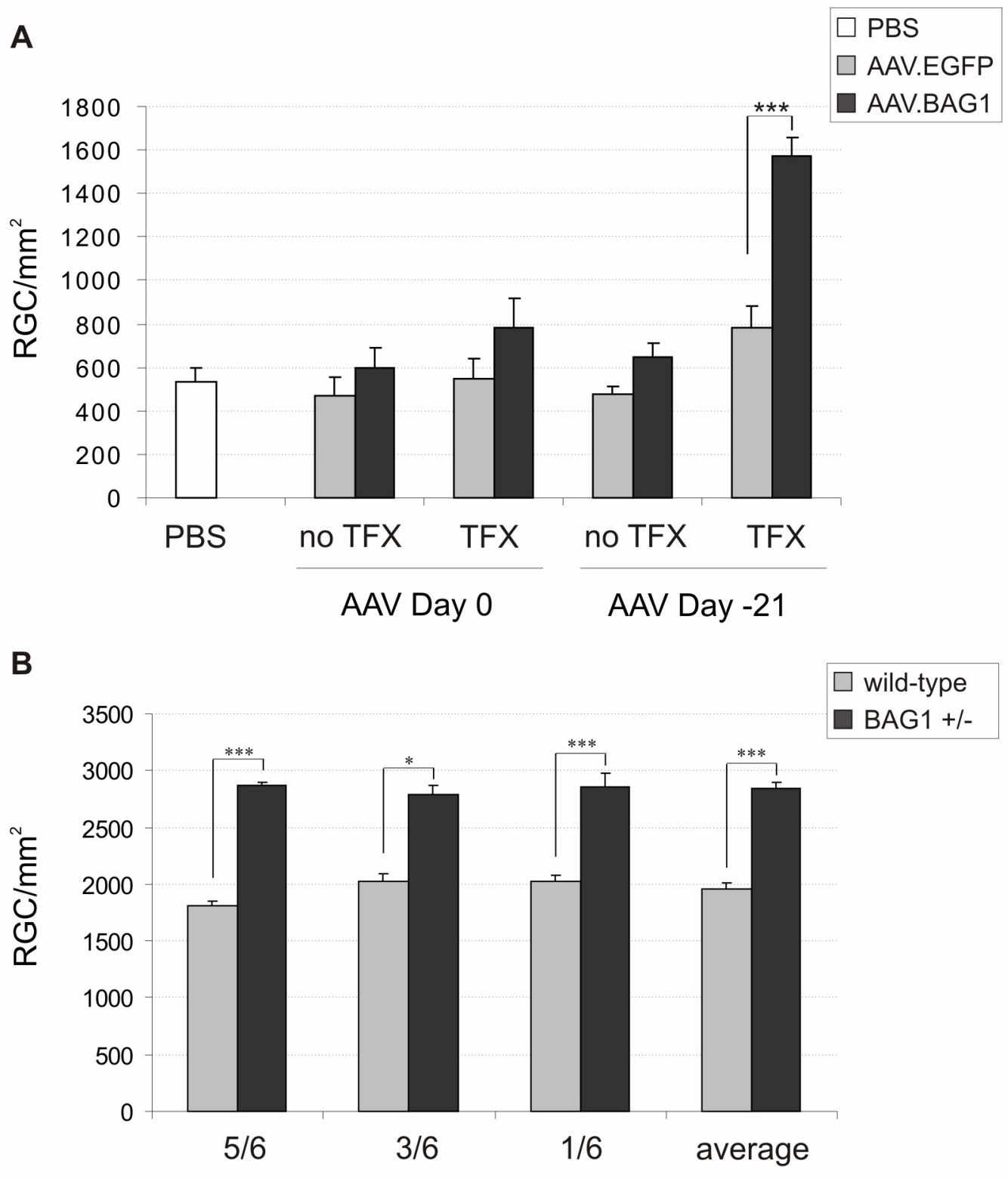

Retinal radius

Figure 16: Quantification of RGC survival after axotomy. A. Surviving RGCs at 14 days post-axotomy in AAV.BAG1 or AAV.EGFP-treated rats. Bars represent means + S.E.M. Group size was as follows: PBS, n=8; AAV.EGFP (d0), n=4; AAV.BAG1 (d0), n=3; AAV.EGFP (d-21), n=5; AAV.BAG1 (d-21), n=6. no TFX, non-transfected area; TFX, transfected area. B. Surviving RGCs in wild-type and BAG1 ${ }^{+/-}$transgenic mice at 14 days post-axotomy at different retinal radiuses. Note that the sizes of RGCs and retinas significantly vary among species. Bars represent means + S.E.M. Wild-type, $n=3 ; \mathrm{BAG1}^{+-}, \mathrm{n}=4{ }^{*}, \mathrm{p}<0.05 ; * * *, \mathrm{p}<0.001$.

\subsubsection{BAG1 increases number and length of regenerating axons after optic nerve crush}

Since BAG1 is known to promote cell differentiation and neurite elongation in vitro, we investigated whether BAG1 is also able to promote axon regeneration in the non-permissive optic nerve crush model in vivo. Animals were treated with $5 \times 10^{7}$ i.u. of AAV.BAG1 or AAV.EGFP immediately after the optic nerve crush procedure. Since regeneration was quantified after 28 days in this model, transgene expression was sufficient after vector 
injection at the day of crush. Animals were sacrificed and optic nerve sections were immunostained for GAP43 to identify regenerating axons (Figure 17).

\section{GAP43}
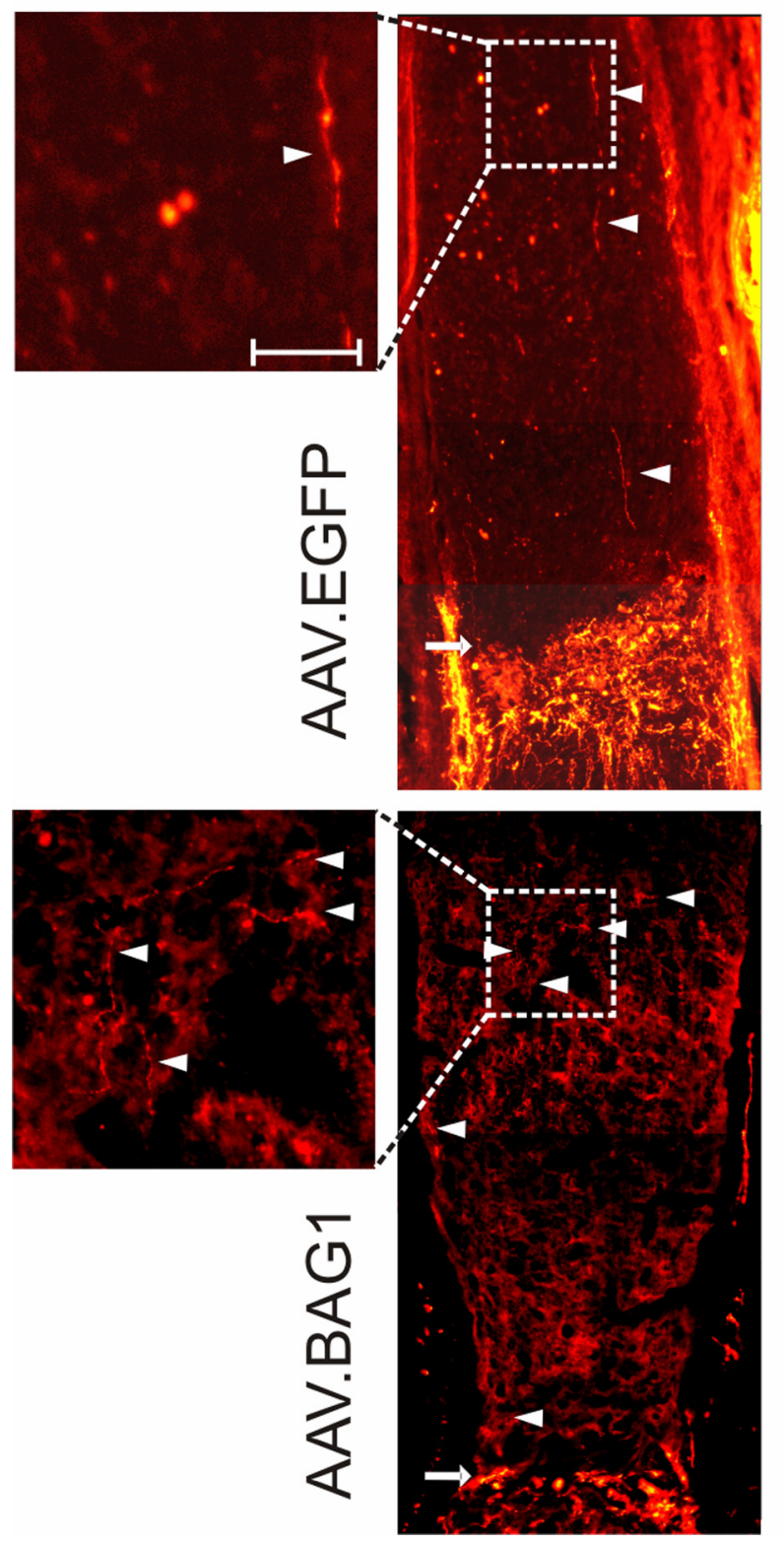

EGFP
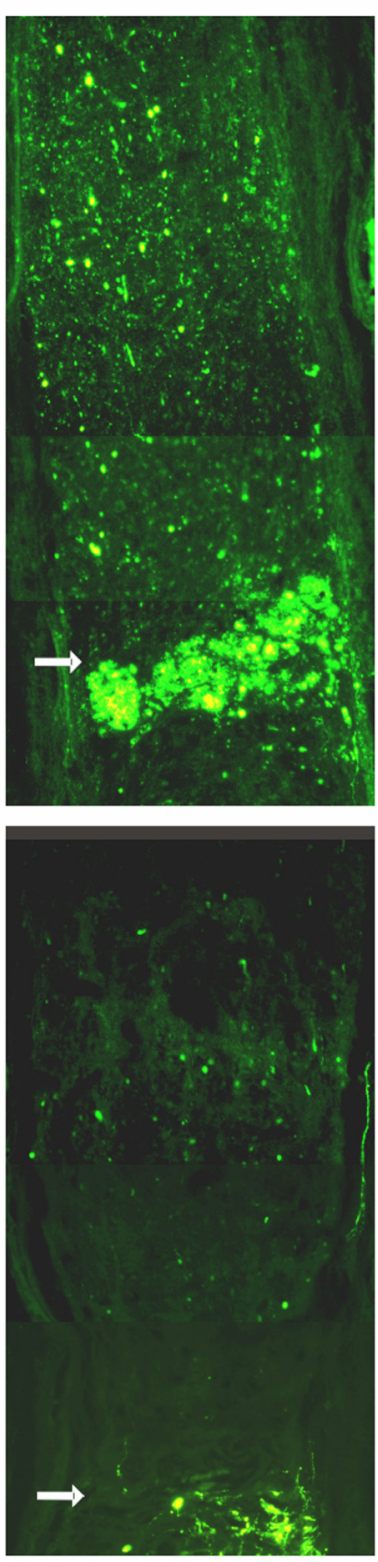

overlay
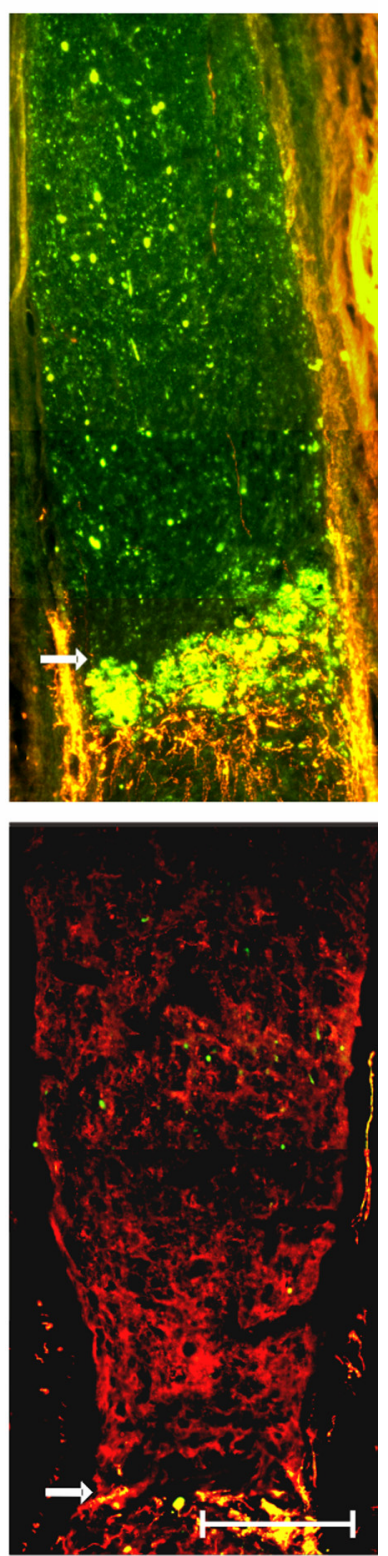

Figure 17: Representative images of regenerating axons in optic nerve sections of animals treated with $5 \times 10^{7}$ i.u. of AAV.EGFP or AAV.BAG1, respectively. Regenerating fibres (stained for GAP43) extend past the crush site (arrows). Arrow heads $(>)$ indicate regenerating fibres extending beyond the crush site. Scale bar: $200 \mu \mathrm{m}$. Scale bar in insert: $50 \mu \mathrm{m}$.

The regenerative response of AAV.EGFP-treated animals did not significantly differ from PBS-treated controls (Figure 18). In contrast, AAV.BAG1 application resulted in a significant increase (up to 3-fold) in number and length of regenerating axons passing the crush site compared with PBS and AAV.EGFP-treated animals. Both PBS and AAV.EGFP control 
animals showed only minor regeneration past the lesion site, while axons regenerating up to $800 \mu \mathrm{m}$ and more past the crush site were observed in animals treated with AAV.BAG1 (Figure 18).

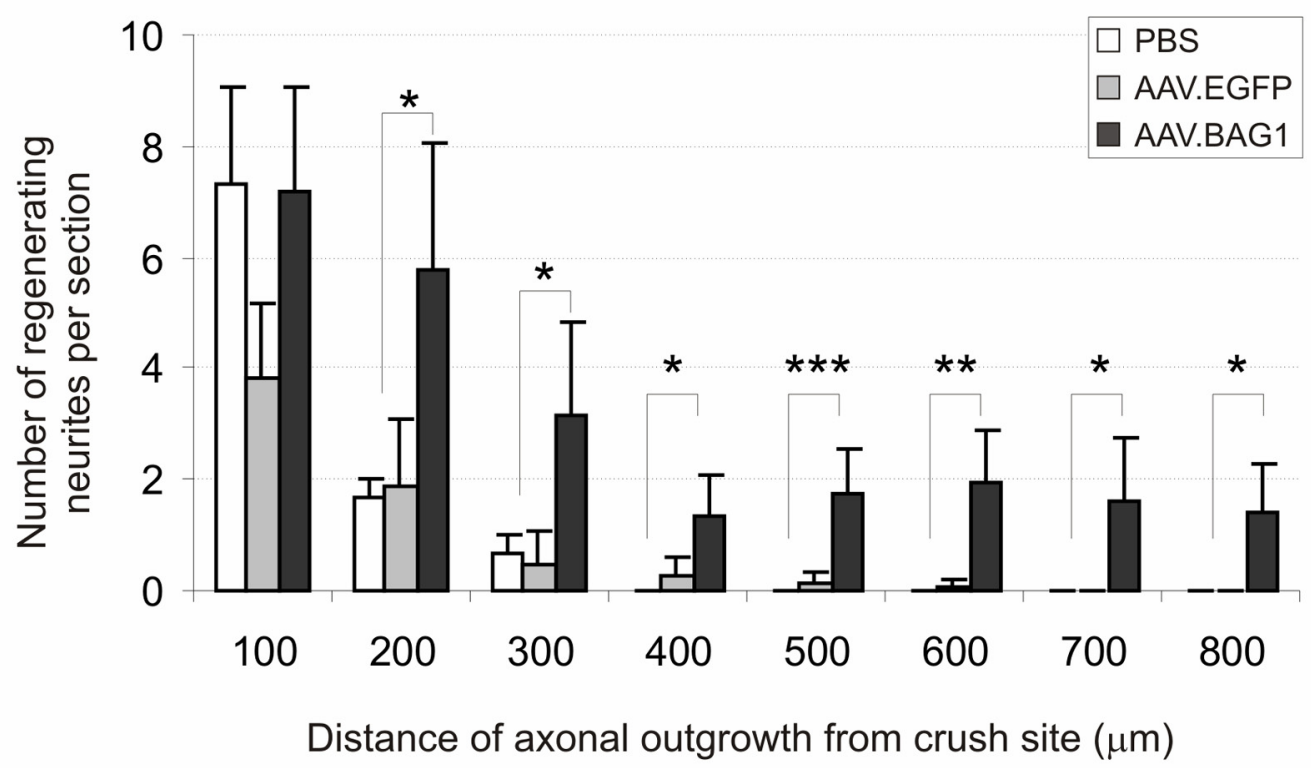

Figure 18: $\quad$ Regenerating axons at day 28 after optic nerve crush at designated distances from the crush site. Bars represent means + S.E.M. Animals groups consisted of: PBS, $n=3$; AAV.EGFP, $\mathrm{n}=4$; AAV.BAG1, $\mathrm{n}=4 .{ }^{*}, \mathrm{p}<0.05 ; * *, \mathrm{p}<0.01 ; * * *, \mathrm{p}<0.001$ (compared to PBS and AAV.EGFP).

\subsection{In vitro studies: Effects of BAG1 overexpression on primary RGCs}

Our in vivo data suggest neuroprotective and regeneration-promoting roles for BAG1 in lesioned RGCs, but the underlying molecular mechanisms so far remained unclear. BAG1 has previously been shown to activate the kinase Raf-1 (Wang et al., 1996; Song et al., 2001). On the other hand, the lack of Raf-1 has been demonstrated to result in recruitment of ROCK2 to the cell membrane and ROCK2 hyperactivity (Ehrenreiter et al., 2005). We thus investigated whether Raf-1-induced translocation of ROCK2 could be involved in BAG1-mediated effects on regeneration.

\subsubsection{Neurite length and growth cone surface of primary RGCs are increased by BAG1}

We first investigated the effects of BAG1 overexpression on primary RGCs. Application of $1.5 \times 10^{8}$ i.u. viral vectors resulted in a transduction efficiency (expression of the EGFP reporter gene) of approximately $70 \%$ of the cells after 5 days (Figure 19.A). Consistent with our in vivo data, application of $1.5 \times 10^{8}$ i.u. of AAV.BAG1 on primary RGCs resulted in a significant increase in neurite outgrowth compared to control cells (Figure 19.A). RGCs 
treated with AAV.BAG1 showed a $\sim 3$-fold higher average neurite length $(651 \pm 160 \mu \mathrm{m})$ compared to AAV.EGFP-treated $(216 \pm 58 \mu \mathrm{m})$ and non-treated control cells $(212 \pm 37 \mu \mathrm{m})$ (Figure 19.B).

In addition, AAV.BAG1-treated RGCs had significantly larger growth cones, exhibiting a larger lamellipodial area and increased number of streak-like point contacts (Figure 20.A).

A

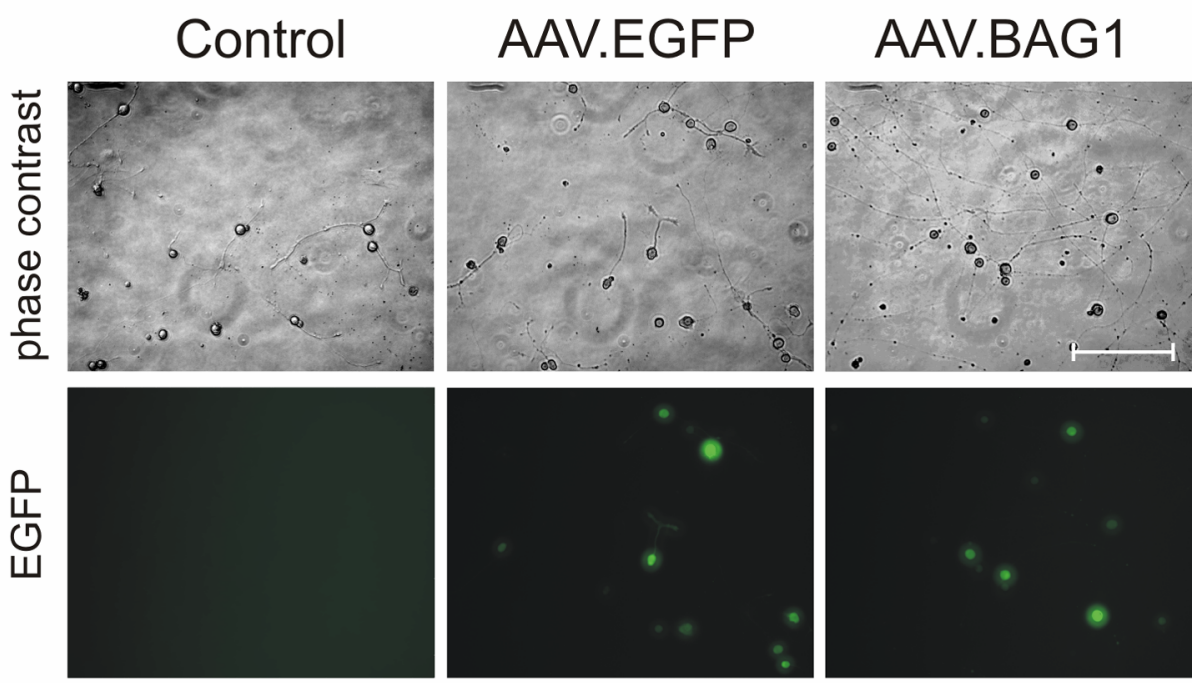

B

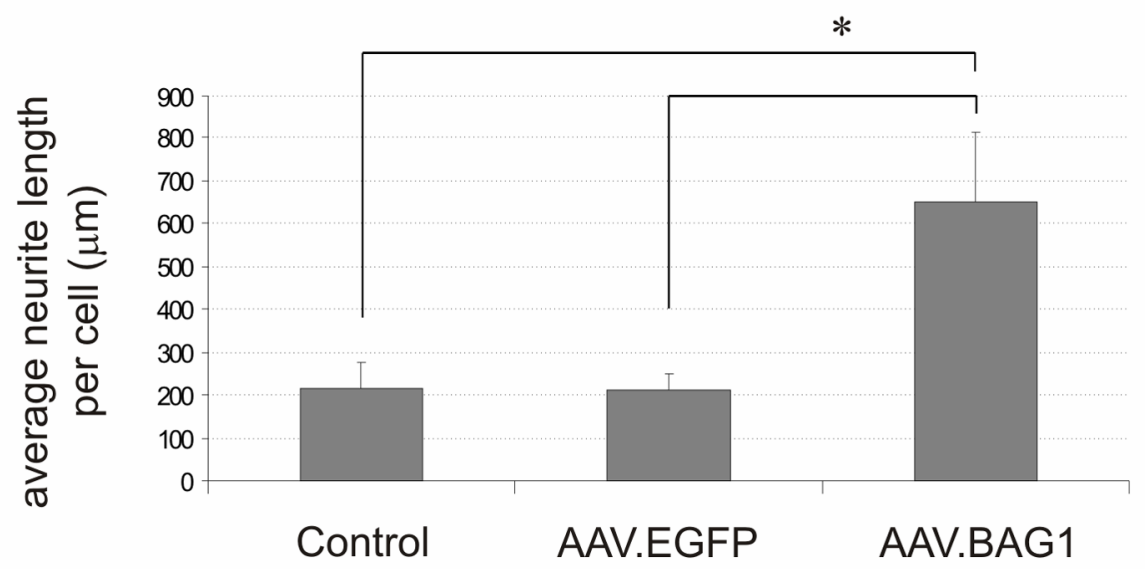

Figure 19: BAG1 increases neurite length in primary RGCs. A. Representative micrographs (phase contrast, upper panel; EGFP, lower panel) of untreated (control), AAV.EGFP and AAV.BAG1-treated primary RGCs. Application of $1.5 \times 10^{8}$ of AAV.BAG1 significantly increases neurite outgrowth after 5 days in culture. Scale bar: $200 \mu \mathrm{m}$. B. Quantitative analysis of neurite outgrowth at 5 days after virus application. Application of AAV.BAG1 resulted in a significant increase in neurite length compared to AAV.EGFP and non-treated cells. Bars represent means + S.E.M. For all treatments, $n=4 .{ }^{*}, \mathrm{p}<0.05$. 
A
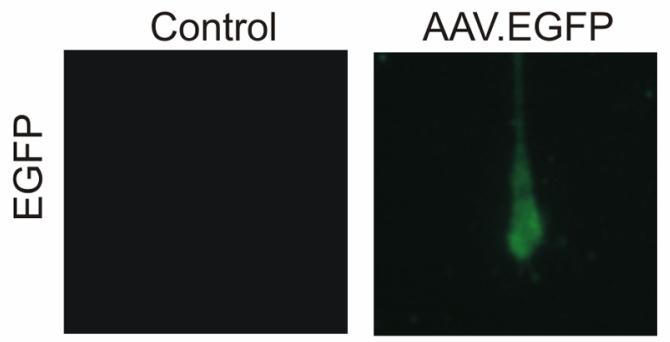

\section{AAV.BAG1}
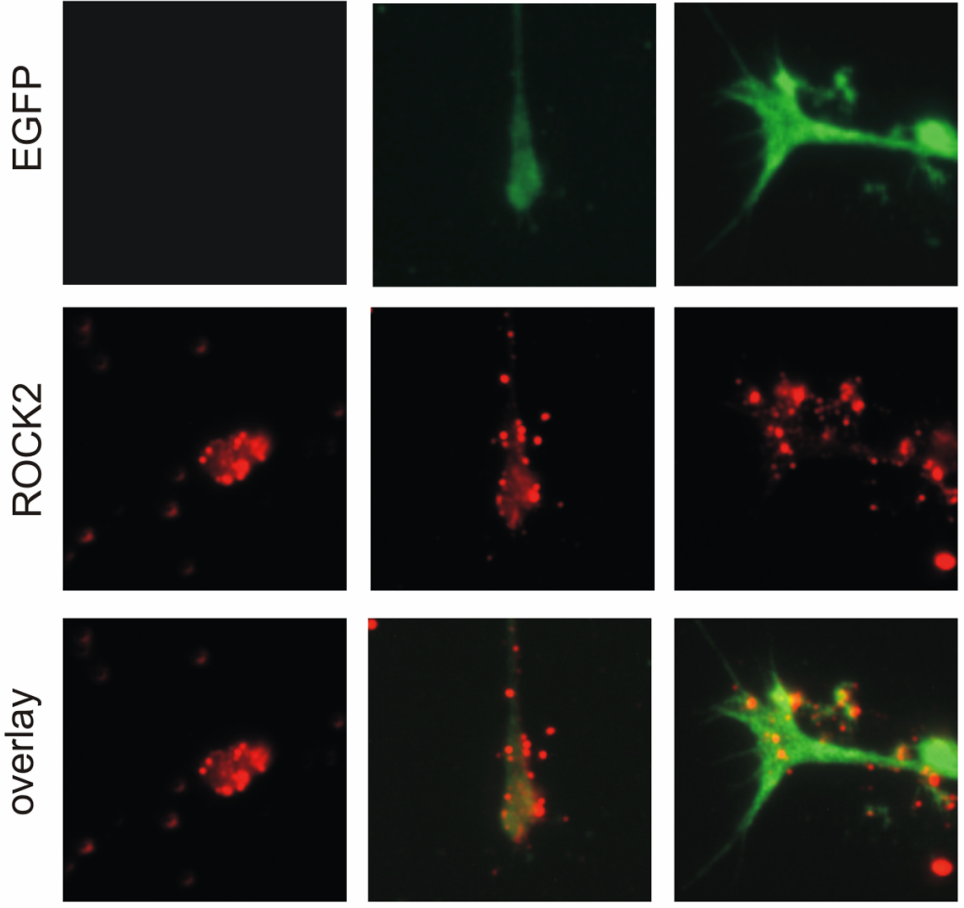

B

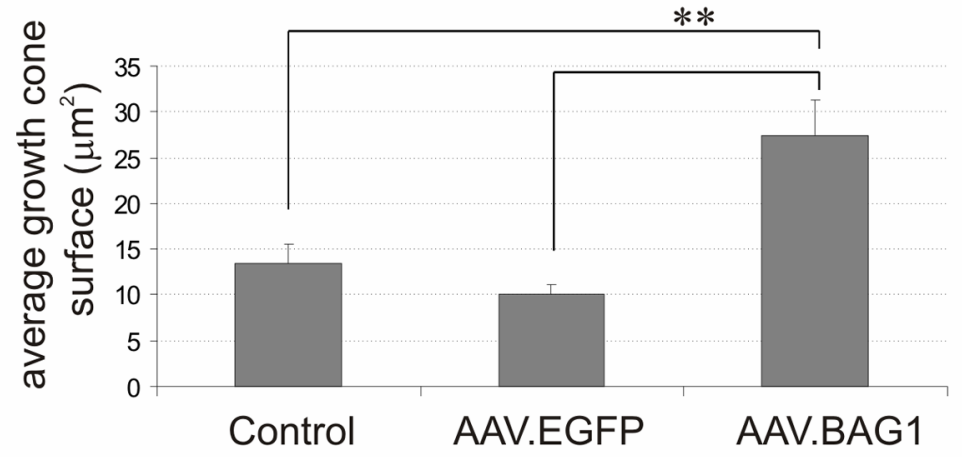

C

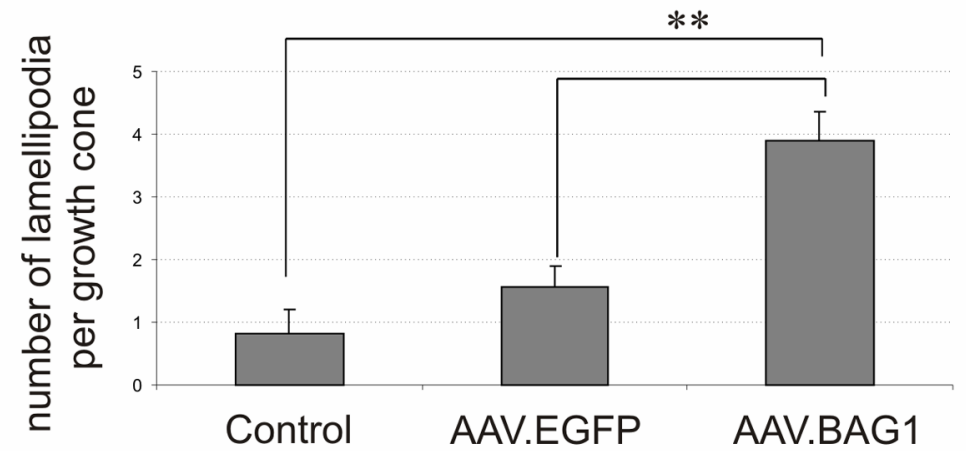

Figure 20: Primary RGC growth cone surface and number of lamellipodia increase upon BAG1 overexpression. A. Representative micrographs of growth cones in primary RGCs treated with AAV.BAG1, AAV.EGFP or in untreated controls. Growth cones are visualized by EGFP expression and ROCK2 (Cy5 filter) immunocytochemistry. B. Quantitative analysis of growth cone surface at 5 days after virus application. Bars represent means + S.E.M. Control, $\mathrm{n}=36$; AAV.EGFP, n=33; AAV.BAG1, n=29. C. Quantitative analysis of number of lamellipodia per growth cone at 5 days after virus application. Bars represent means + S.E.M. Control, $n=27$; AAV.EGFP, $n=33$; AAV.BAG1, $n=31 .{ }^{* *}, \mathrm{p}<0.01$. 
Quantitative analysis of the growth cone area showed a $\sim 2.5$-fold larger average growth cone surface $\left(27.39 \pm 3.81 \mu^{2}\right)$ compared to AAV.EGFP-treated $\left(10.07 \pm 1.07 \mu \mathrm{m}^{2}\right)$ and nontreated control cells $\left(13.37 \pm 2.12 \mu \mathrm{m}^{2}\right.$, Figure 20.B). The number of lamellipodia per growth cone exhibited a similar increase, with an average of $3.9 \pm 0.4$ lamellipodia per growth cone compared to $1.5 \pm 0.3$ and $0.8 \pm 0.4$ in AAV.EGFP-treated and non-treated control cells, respectively (Figure 20.C).

\subsubsection{Translocation of Raf-1 and ROCK2 in primary RGCs after BAG1 overexpression}

We then examined the subcellular localisation of Raf-1 and ROCK2 upon BAG1 overexpression. Primary RGCs were immunostained against Raf-1 and ROCK2. "Pseudo-

A AAV.EGFP
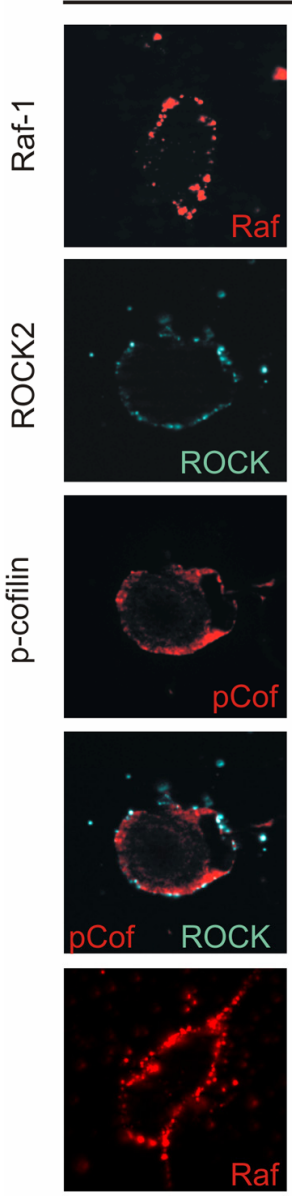
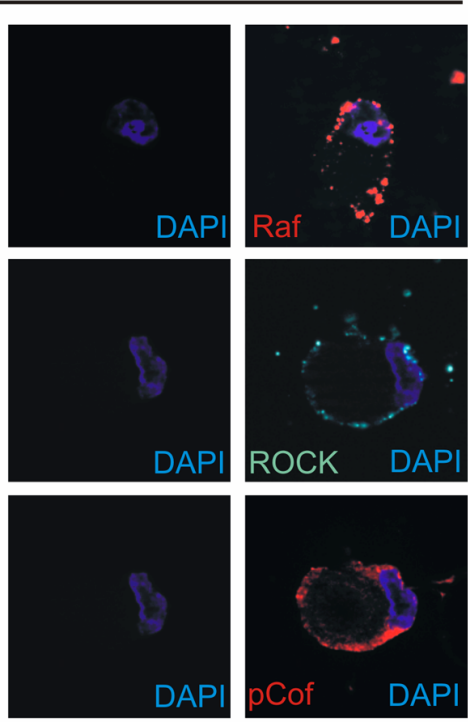

DAPI
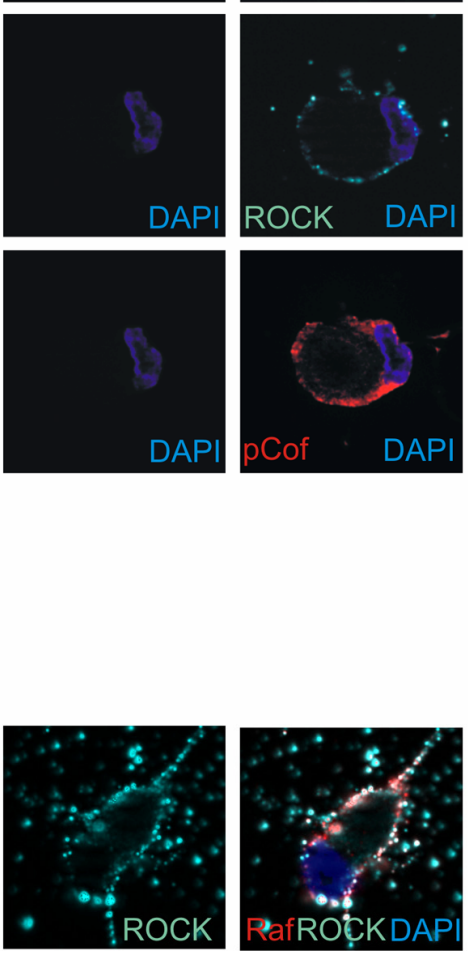

B

AAV.BAG1
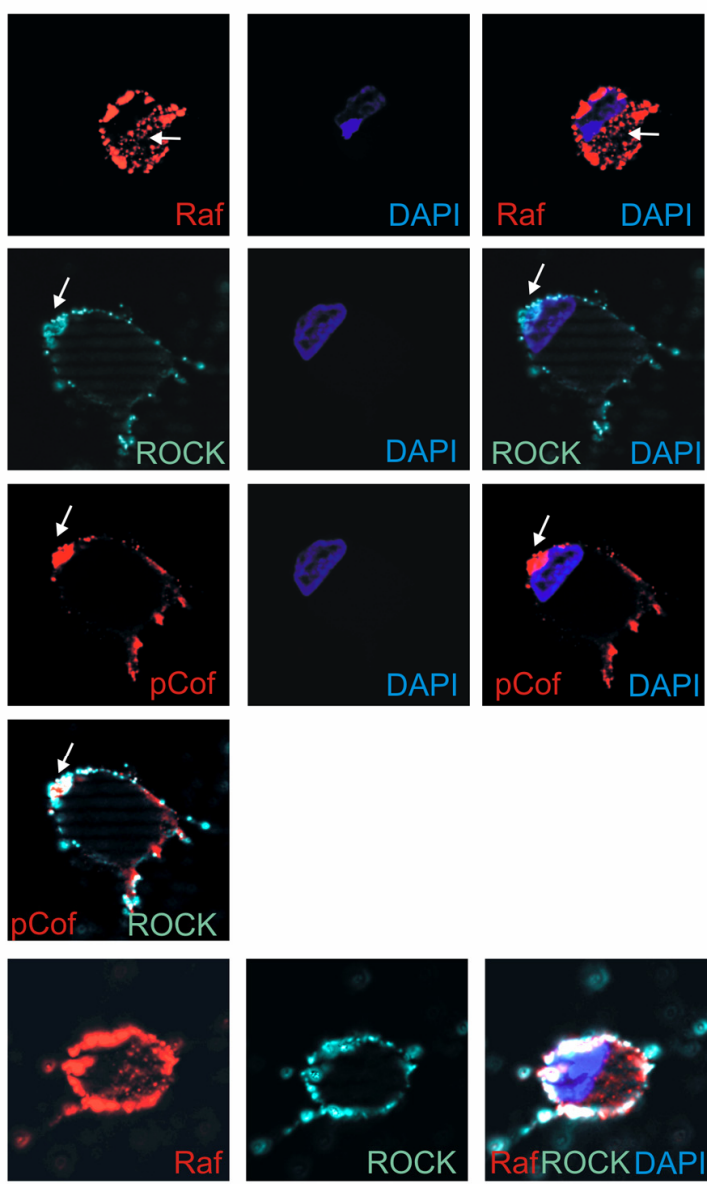

Figure 21: Photomicrographs depicting subcellular localisation of Raf-1, ROCK2 and phosphocofilin in primary RGCs. Cells were treated with $1.5 \times 10^{8}$ i.u. AAV.EGFP (A) and AAV.BAG1 (B) respectively for 5 days and stained with antibodies specific for Raf-1, ROCK2 and phospho-cofilin followed by the appropriate $\mathrm{Cy} 3$-anti-mouse, Cy5-anti-goat and $\mathrm{Cy} 3$-antirabbit antibodies and nuclear staining (DAPI). Arrows indicate translocation of Raf-1 to the cytoplasmic compartment (upper row) and translocation of ROCK2 to the nuclear/perinuclear space (rows 2-4) in AAV.BAG1-treated cells as compared to AAV.EGFP treated controls. Raf-1 and ROCK2 partially colocalise (row 5). 
confocal" Fluorescence microscopy (using a Zeiss Apotome ${ }^{\mathrm{TM}}$ device) of AAV.EGFP-treated cells showed a membrane-bound localisation of both Raf-1 and ROCK2 (Figure 21). In contrast, AAV.BAG1 induced a more cytoplasmic immunoreactivity of Raf-1. Costaining with DAPI and anti-phospho-cofilin revealed that Raf-1 was not translocated to the nucleus, but indeed localised in the cytoplasm upon AAV.BAG1 application (Figure 21, row 3). In both AAV.EGFP and AAV.BAG1-treated RGCs, phospho-cofilin was mostly localised at the membrane. ROCK2 partially colocalised with phospho-cofilin (Figure 21, row 4). Interestingly, AAV.BAG1-treated cells showed a strong perinuclear concentration of ROCK2 compared to AAV.EGFP-treated controls. Co-staining with Raf-1 and ROCK2 showed a colocalisation of both proteins mostly at the membrane (Figure 21, lower row), consistent with a previous report (Ehrenreiter et al., 2005).

\subsection{In vitro studies: Effects of BAG1 overexpression in CSM cells}

To better examine subcellular protein localisation, we used the rat neuronal CSM14.1 cell line stably transfected with mouse Flag-BAG1 (Kermer et al., 2002). CSM14.1 cells have larger cell bodies than RGCs, allowing for a better identification of cellular structures and the generation of sufficient amounts of protein lysates to perform immunoblots. Upon growth at a permissive temperature $\left(32^{\circ} \mathrm{C}\right), \mathrm{CSM}$ cells remain in a non-differentiated state and proliferate. Incubation at a non-permissive temperature $\left(39^{\circ} \mathrm{C}\right)$ permits their differentiation into neurons.

\subsubsection{BAG1 induces Raf-1 and ROCK2 translocation in CSM cells: western blot analysis}

To better quantify the subcellular localisation of Raf-1 and ROCK2, lysates from wild-type or BAG1 overexpressing CSM cells were analysed by Western blot and probed against BAG1, Raf-1, ROCK2 and RhoA. Endogenous (non-flag-tagged) BAG1 expression showed no significant difference between wild-type and BAG1 overexpressing cells, both at permissive (Figure 22.A) and non-permissive (Figure 22.B) temperatures. BAG1 expression in nondifferentiated cells (Figure 22.A) was slightly stronger in the nucleus, while it remains more cytosolic in differentiated cells (Figure 22.B) when compared to membrane expression. These results are in line with previous works reporting a shift from nuclear to cytosolic localisation of BAG1 upon differentiation (Kermer et al., 2002). Raf-1 expression levels in cytosolic, membrane and nuclear fractions did not significantly differ between wild-type and BAG1 overexpressing cells or between the two differentiation states. However, in the cytoskeletal fraction, Raf-1 expression pattern strongly varied upon differentiation. Non-differentiated 
wild-type cells show a much higher Raf-1 protein level than BAG1 overexpressing cells (Figure 22.A), while differentiated BAG1 cells exhibit higher Raf-1 levels than wild-type cells (Figure 22.B). This suggests a shift of Raf-1 to the cytoskeleton in differentiated cells upon BAG1 overexpression, which confirms the results obtained in primary RGC cultures (Figure $22 \mathrm{~A}$ and B). Western blots of ROCK2 show a higher expression level in the nuclear fractions upon BAG1 overexpression, independent of the cell differentiation state (Figure 22. A and B). In addition, a weak band is detected in the cytoskeletal fraction of differentiated wild-type cells only, while BAG1 overexpressing cells fail to show any cytoskeletal ROCK2 expression. In accordance to the results obtained in primary RGCs, ROCK2 expression shifted to the nuclear fraction in differentiated BAG1 overexpressing CSM cells (Figures 22 and 21.B).

A

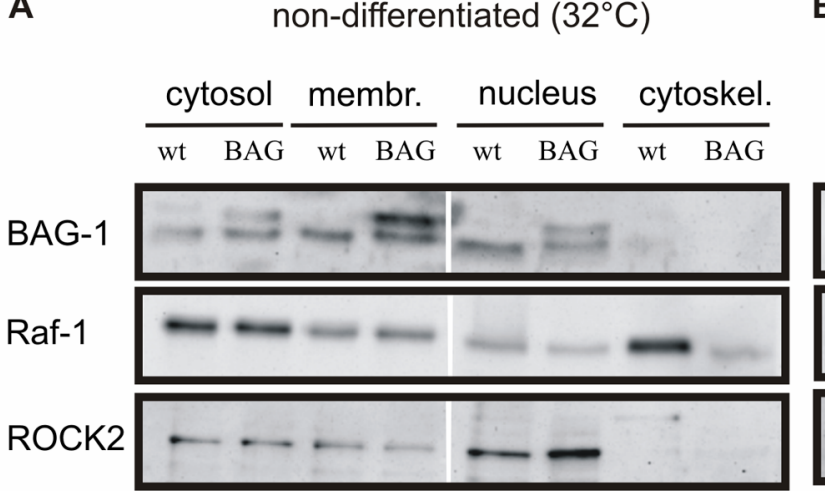

B differentiated $\left(39^{\circ} \mathrm{C}\right)$

Figure 22: Representative western blots of subcellular expression of BAG1, Raf-1, and ROCK2 in non-differentiated (A) and differentiated (B) CSM cells respectively. Experiments were done at least in triplicates from cell lysates from 3 different experiments and cell passages. Endogenous expression of BAG1 (lower band in BAG1 line) was used as a loading control. wt, wild-type CSM cells; BAG, BAG1 overexpressing CSM cells. Size standards in kDa are shown on the right. Note that it is not possible to compare protein expression among blots (A versus B, and between specific antibodies) because of the different exposure times used.

To exclude that subcellular translocations are due to changes in overall expression and further examine possible effects of BAG1 overexpression on the Raf-1 and ROCK-signalling cascades, we analysed protein expression in whole cell lysates. Figure 23 shows the expression levels of Raf-1, phospho-Raf-1, the downstream effectors of the Ras-Raf-pathway ERK, phospho-ERK and phospho-JNK, RhoA and ROCK2, in non-differentiated and differentiated CSM respectively. 
A

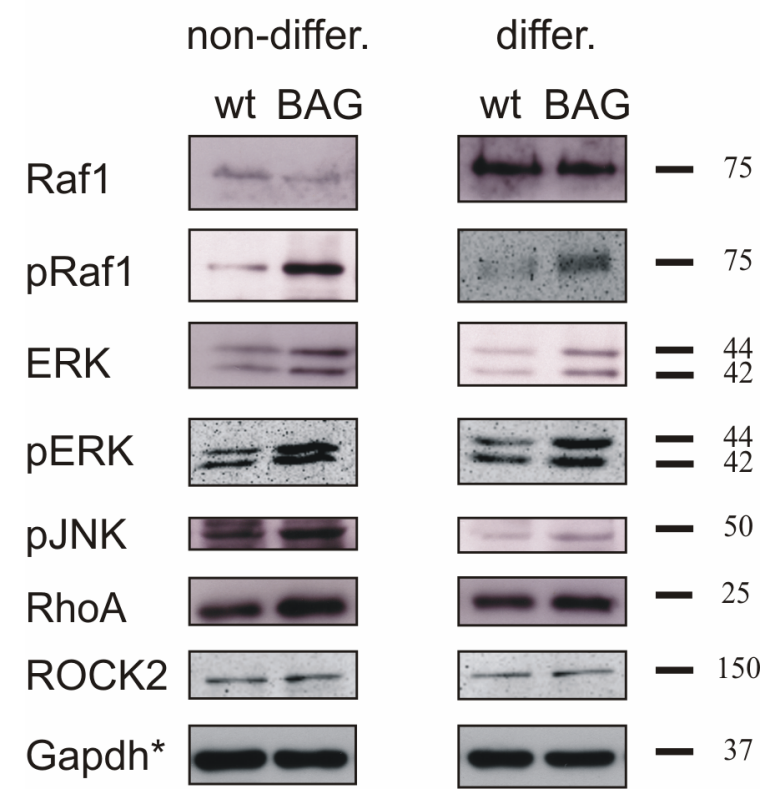

Figure 23: Western blot analysis of downstream effectors of the Ras-Raf-MAPK cascade in nondifferentiated (A) and differentiated (B) CSM cells, respectively. Experiments were repeated at least 3 times with cell lysates from 3 different experiments and cell passages. Glyceraldehyde-3-phosphate dehydrogenase (Gapdh) was probed for each western blot as a loading control. *, representative picture; non-differ., non-differentiated cells; differ., differentiated cells; wt, wild-type CSM cells; BAG, BAG1 overexpressing CSM cells. Size standards in $\mathrm{kDa}$ are shown on the right. Note that it is not possible to compare protein expression among blots because of the different exposure times used.

BAG1 overexpression induced a significant increase in phospho-Raf-1 expression, while Raf1 levels remained stable. These results are in line with previous works reporting Raf-1 activation (e.g. phosphorylation) upon BAG1 stimulation (Wang et al., 1996; Song et al., 2001). ERK and phospho-ERK levels were also increased in BAG1 overexpressing cells (Kermer et al., 2002). Levels of phospho-JNK show a moderate increase upon BAG1 overexpression. Western blots of RhoA showed no significant differences in expression levels between differentiated wild-type and BAG1 overexpressing cells. However, RhoA levels were significantly higher in non-differentiated BAG1 overexpressing cells. No significant differences were observed in ROCK2 expression levels between wild-type and BAG1 overexpressing cells.

\subsubsection{BAG1 induces Raf-1 and ROCK2 translocation in CSM cells: immuno-cytochemical analysis}

For colocalisation studies, non-differentiated and differentiated CSM cells were immunostained with rhodamine-conjugated phalloidin, a marker for F-actin, and antibodies specific for Raf-1 and ROCK2 and visualised by confocal microscopy (Figure 24). 
A

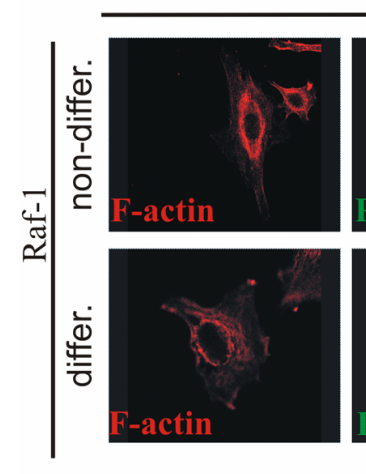

wild-type
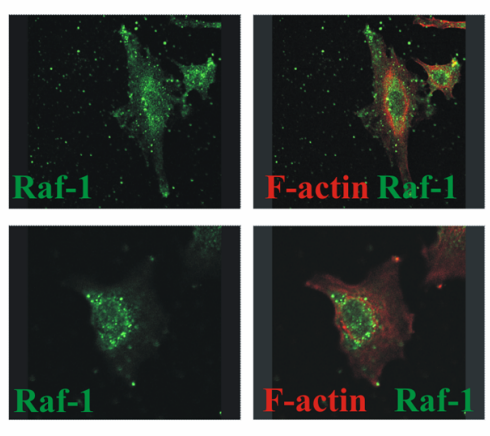

B
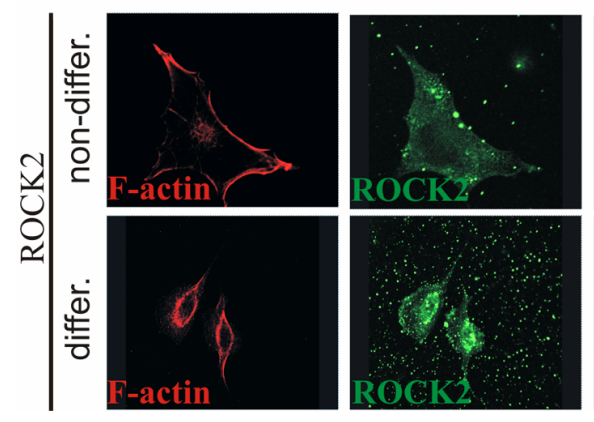
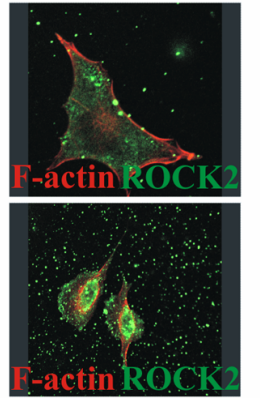

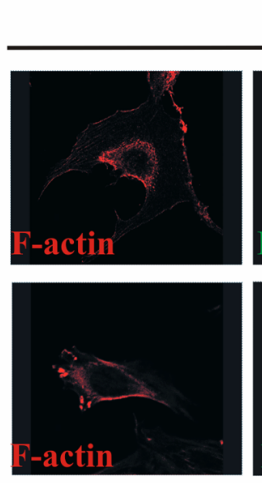

BAG
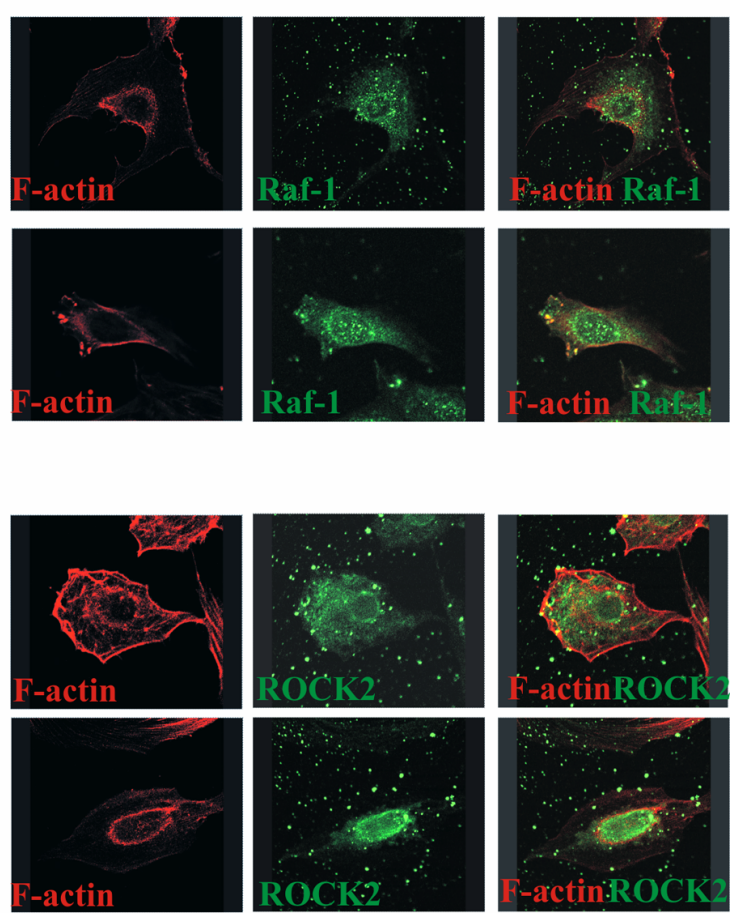

Figure 24: Cellular localisation of Raf-1 (A) and ROCK2 (B) in non-differentiated and differentiated wild-type and BAG1 overexpressing (BAG) CSM cells. Cells stained with rhodamineconjugated phalloidin and antibodies specific for Raf-1 and ROCK2 followed by the appropriate Cy5-anti-mouse and Cy5-anti-goat antibodies were analysed by confocal microscopy. non-differ., non-differentiated cells; differ., differentiated cells.

Raf-1 expression did not show any change upon differentiation state or upon BAG1 overexpression and showed no colocalisation with phalloidin. ROCK2 did not colocalise with F-actin. Consistent with our results from western blot and primary RGCs, ROCK2 expression was more visible in the perinuclear and nuclear space in BAG1 overexpressing cells.

\subsection{In vitro studies: ROCK activity in CSM cells}

\subsubsection{ROCK activity is decreased upon BAG1 overexpression}

The lack of Raf-1 has been previously demonstrated to result in the recruitment of ROCK2 to the cell membrane and increase of ROCK2 activity (Ehrenreiter et al., 2005). Accordingly, our in vitro results showed that overexpression of BAG1 induced a differential translocation of Raf-1 and ROCK2. Most importantly, however, we wanted to know whether overexpression of BAG1 can influence ROCK activity in addition to its effect on protein localisation. We therefore quantified ROCK activity by ELISA in whole lysates of BAG1 overexpressing or wild-type CSM cells. Figure 25 shows the averaged ROCK activity of three 
independent experiments expressed in percent of wild-type controls. ROCK activity of nondifferentiated cells does not significantly differ between BAG1 overexpressing cells and wildtype controls. In contrast, overexpression of BAG1 in differentiated neuron-like cells significantly reduced ROCK activity by about $50 \%$ compared to wild-type cells.

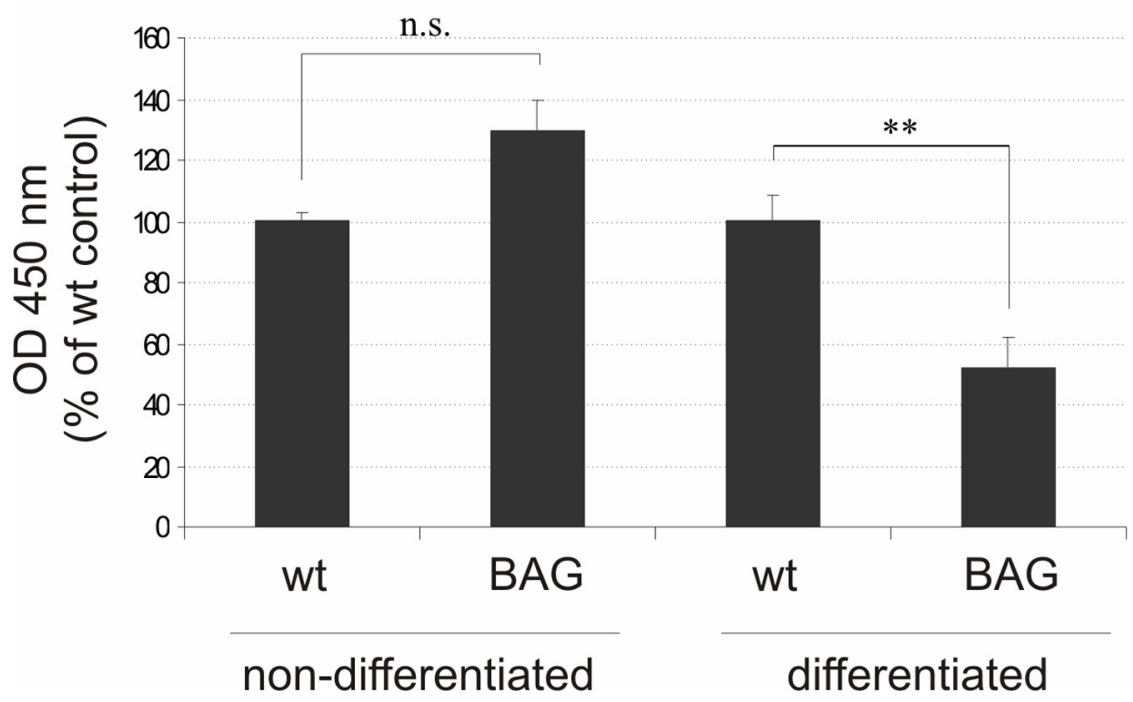

Figure 25: $\quad$ ROCK activity in wild-type (wt) and BAG1 overexpressing (BAG) CSM cells. Data are from 3 independent experiments each. Bars represent means + S.E.M. n.s., not significant; **, $\mathrm{p}<0.01$.

\subsubsection{BAG1-mediated decrease in ROCK activity is Raf-1-dependent}

Since BAG1 is known to activate Raf-1 and we could show both a translocation and activation of Raf-1 upon BAG1 overexpression, we further examined whether Raf-1 could be the molecular link between BAG1 overexpression and the observed decrease in ROCK activity. For this purpose, we used the Raf-1 inhibitor GW5074, which is reported to specifically inhibit the Raf-1 kinase (Lackey et al., 2000; Chin et al., 2004). CSM cells were treated for $24 \mathrm{~h}$ with GW5074 or DMSO before lysis and Raf-1 inhibition was verified by western blot analysis. Figure 26 shows expression levels of phospho-Raf-1, phospho-ERK and ROCK2 in differentiated wild-type and BAG1 overexpressing CSM cells. Inhibitor application resulted in a decrease of phospho-Raf-1 and its activated downstream effector phospho-ERK compared to DMSO-treated controls, demonstrating the efficient inhibition of Raf-1. ROCK2 expression was not influenced by Raf-1 inhibition. 


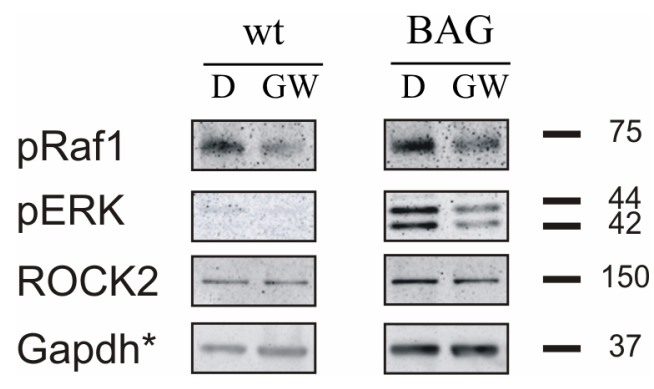

Figure 26: Western analysis of phospho-Raf-1, phospho-ERK and ROCK2 expression following Raf1 kinase inhibition. Application of $500 \mathrm{nM}$ of Raf-1 kinase inhibitor GW5074 (GW) leads to a decrease of phospho-Raf-1 expression and its activated downstream effector phospho-ERK compared to DMSO-treated control (D). Experiments were repeated at least 3 times with cell lysates from 3 different experiments and cell passages. Gapdh was probed for each western blot as a loading control. Size standards in $\mathrm{kDa}$ are shown on the right. wt, wild-type CSM cells; BAG, BAG1 overexpressing CSM cells; *, representative picture.

ROCK kinase activity assay was then quantified in lysates of differentiated cells treated with the Raf-1 kinase inhibitor. Application of GW5074 resulted in a concentration-dependent increase in ROCK activity, both in wild-type and BAG1 overexpressing CSM cells (Figure 27). Incubation with $2000 \mathrm{nM} \mathrm{GW5074} \mathrm{significantly} \mathrm{increased} \mathrm{ROCK} \mathrm{activity,} \mathrm{resulting} \mathrm{in} \mathrm{a}$ significantly higher enzymatic activity in wild-type and BAG1 overexpressing CSM cells compared to DMSO controls $(138.5 \pm 8.6 \%$ vs. $100.0 \pm 4.3 \%$ and $88.3 \pm 6.2 \%$ vs. $66.3 \pm$ $5.9 \%$, respectively).

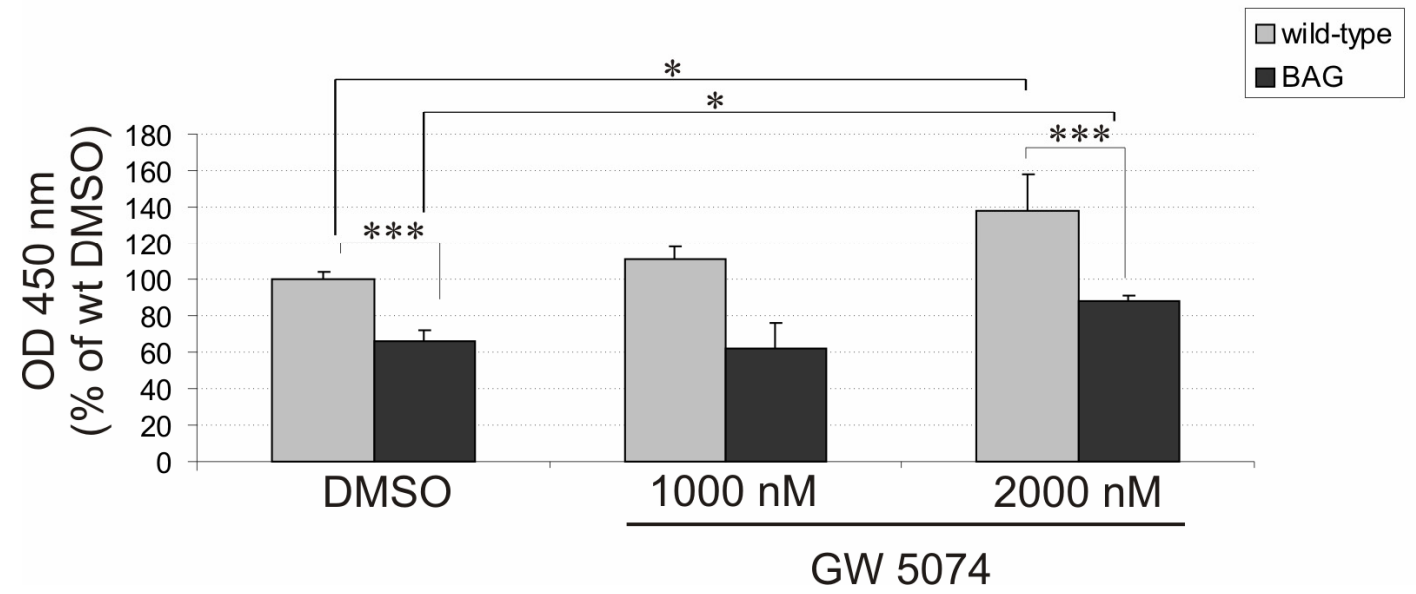

Figure 27: Inhibition of Raf-1 kinase activity in differentiated wild-type and BAG1 overexpressing (BAG) CSM cells affects ROCK activity in a dose-dependent manner. Data are from at least 3 independent experiments each. Bars represent means + S.E.M. * ${ }^{*}<0.05 ; * * *, p<0.001$.

To confirm that Raf-1 is the molecular link between BAG1 overexpression, decrease in ROCK activity and enhanced neurite outgrowth, we tested whether application of GW5074 had an impact on the neurite outgrowth of primary RGCs. Cells transfected with AAV.EGFP 
or AAV.BAG1 were treated for 3 days with $500 \mathrm{nM}$ DMSO or GW5074 respectively.

Application of Raf-1 inhibitor showed no obvious cell toxicity and cell density was preserved.

A

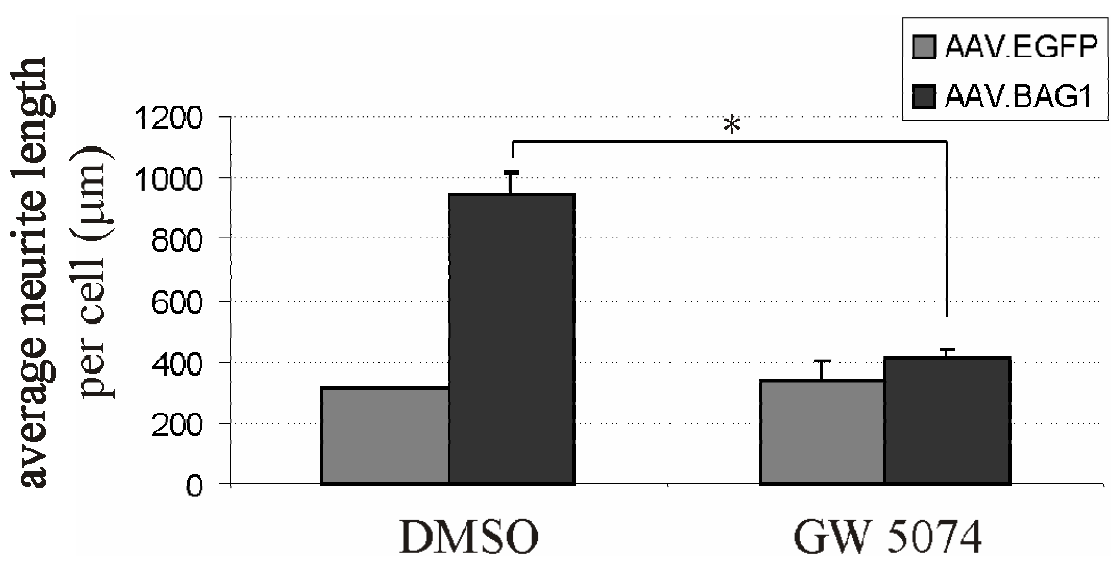

B

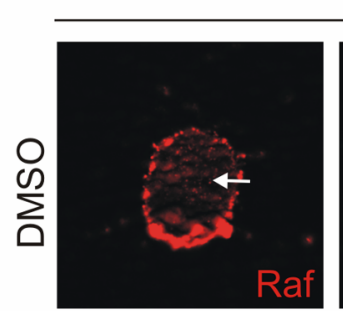

AAV.BAG1
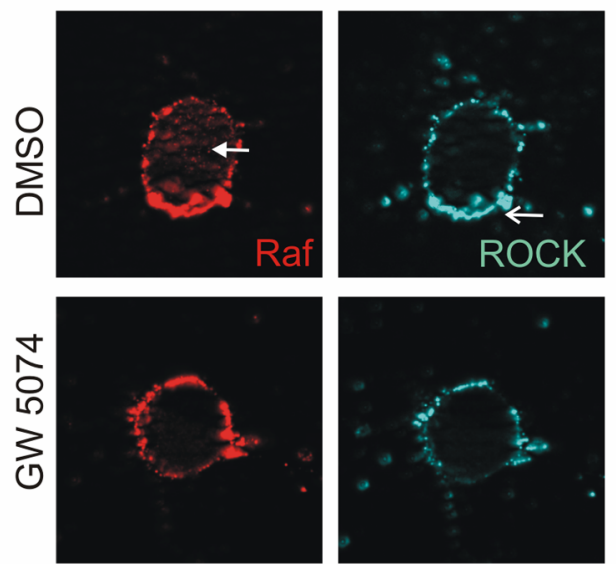

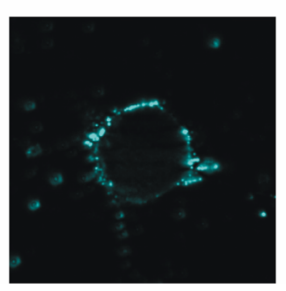

C

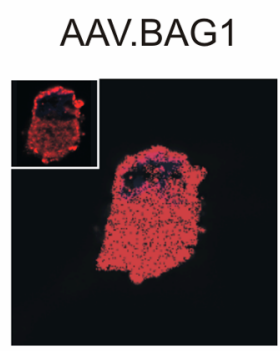

AAV.EGFP
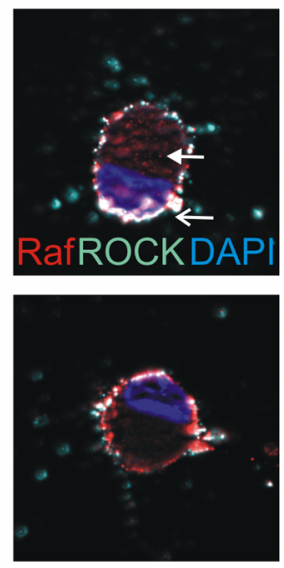

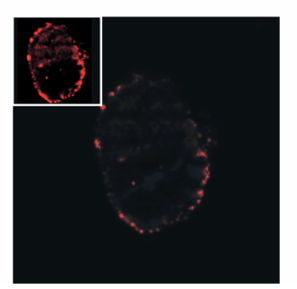

Figure 28: Raf-1 inhibition reverses the effects mediated by BAG1. A. Quantification of neurite length in primary RGCs after treatment with the Raf-1 inhibitor GW5074 or DMSO (control). AAV.BAG1-transduced RGCs have longer neurites than AAV.EGFP-treated controls and this effect of BAG1 is reversed by treatment with the Raf-1 inhibitor GW5074. *, p $<0.05$. B. Photomicrographs depicting Raf-1 and ROCK2 subcellular localisation in primary RGCs treated with AAV.BAG1 and DMSO (control) or Raf-1 inhibitor (GW5074). Cells were stained with antibodies specific for Raf-1 and ROCK2 followed by the appropriate Cy3-antimouse and Cy5-anti-goat antibodies and DAPI. Arrows indicate translocation of Raf- 1 to the cytoplasmic compartment and ROCK2 to the nuclear/perinuclear space only in the control condition (DMSO-treated), while GW5074 treatment reverses the BAG1-induced translocation of Raf-1 and ROCK2. C. Expression of phospho-ERK in primary RGCs transduced with AAV.EGFP or AAV.BAG1 and treated with DMSO and GW5074 respectively. Cells were stained for phospho-ERK and pictures taken with the same settings $(63 \mathrm{x}$ oil immersion objective, Cy-3 filter, exposure time $6 \mathrm{sec}$ ). Pictures in inserts were taken with optimal (variable) exposure times. Application of Raf-1 inhibitor significantly reduces phospho-ERK levels.

Upon DMSO treatment, AAV.BAG1-treated cells exhibited a $\sim 3$-fold higher average neurite length compared to AAV.EGFP $(942.4 \pm 74.6 \mu \mathrm{m}$ and $314.8 \pm 0.4 \mu \mathrm{m}$ respectively, Figure 
28.A). In contrast, application of $500 \mathrm{nM} \mathrm{GW5074} \mathrm{dramatically} \mathrm{impaired} \mathrm{neurite} \mathrm{outgrowth}$ (AAV.BAG1: $411.1 \pm 32.6 \mu \mathrm{m}$; AAV.EGFP: $337.6 \pm 70.2 \mu \mathrm{m}$ ). Analysis by "pseudoconfocal" fluorescence microscopy (using the Apotome ${ }^{\mathrm{TM}}$ device) of co-staining with Raf-1 and ROCK2 showed that following GW5074 application, AAV.BAG1-treated cells exhibit a Raf-1 and ROCK2 membrane-bound localisation similar to AAV.EGFP-treated controls (Figure 28.B; compare with Figure 21). Additional staining of phospho-ERK showed a marked decrease in phospho-ERK levels following application of the Raf-1 inhibitor, confirming the efficient inhibition of Raf-1 in our culture model (Figure 28.C). 


\section{Discussion}

Regenerative failure in the adult CNS causes persistent neurological deficits in degenerative, inflammatory and traumatic neurological disease. Successful regenerative strategies for the CNS depend on the simultaneous targeting of different mechanisms, such as lesion-induced neuronal cell death and growth inhibitory signals derived from myelin proteins. In the present work, we have evaluated a gene therapeutic approach to counteract apoptosis and growth inhibition by AAV-mediated overexpression of BAG1 in RGCs.

We investigated the effects of BAG1 overexpression in two models of apoptosis and regeneration of the retinal ganglion cell system in vivo, the optic nerve axotomy and the optic nerve crush. We show that BAG1 overexpression significantly protects RGCs from axotomyinduced cell-death and promotes RGC regeneration in non-permissive conditions, and establish a new signalling cascade that mediates the effects of BAG1 in regeneration.

\subsection{BAG1 is neuroprotective in vivo}

The co-chaperone BAG1 has previously been shown to act in an anti-apoptotic and neuroprotective manner, i.e. in an experimental stroke model in vivo (Kermer et al., 2003). Here, we have used the optic nerve axotomy model to assess neuroprotection after traumatic injury. In previous studies, our laboratory could demonstrate that more than $90 \%$ of RGCs degenerate two weeks following deafferentiation and that this model is suitable to examine neuroprotective effects in vivo (Kermer et al., 1998; Lingor et al., 2005; Malik et al., 2005). In our experimental setup, the AAV vector was applied three weeks prior to surgery $(\mathrm{d}-21)$, allowing for sufficient protein expression before lesioning, or at the time of axotomy (d 0 ), mimicking a possible therapeutic application. Although sufficient retinal transgene expression was observed in both groups at the time of extraction of the retinas (d 14), only the group receiving the AAV.BAG1 injection prior to the lesion showed a significant increase $(\sim 70 \%)$ in surviving RGC numbers compared to the AAV.EGFP controls (Figure 16.A). Transgene expression under the human synapsin promoter from AAV vectors requires several days to fully express the transgene in infected neurons (Kügler et al., 2003b). It may therefore not be sufficient to counteract apoptosis when the vector is applied simultaneously with the lesion. The moderate, but not significant, increase in the number of surviving RGCs observed in AAV.EGFP-transfected areas may be due to cytokine secretion following the virus application itself (Straten et al., 2002). Although other studies have failed to report a 
cytoprotective effect of AAV by itself (Malik et al., 2005), larger numbers of animals would have to be analyzed to evaluate putative effects of AAV on cell survival. Nevertheless, while the effects of AAV.EGFP on RGC survival were not significant, AAV.BAG1 markedly increased RGC number following axotomy. This was confirmed in $\mathrm{BAG} 1^{-/+}$transgenic mice, which showed a $\sim 50 \%$ increase in RCG survival compared to wild-type littermates (Figure 16.B).

\subsection{BAG1 promotes regeneration in vivo}

In addition to its neuroprotective effects, overexpression of BAG1 in vitro results in longer neurites and increased generation time in CSM cells (Kermer et al., 2002). In our in vivo regeneration model, RGC regeneration was quantified 28 days after crush. Here, transgene expression after vector injection at the day of lesion (d 0) was sufficient to significantly enhance axon regeneration in AAV.BAG1-treated animals, opening a window for future therapeutic options. BAG1 overexpression resulted in an up to 3-fold higher number of regenerating axons extending $800 \mu \mathrm{m}$ and more past the crush site compared to animals treated with PBS or AAV.EGFP, which showed a maximal regeneration length of $600 \mu \mathrm{m}$ (Figure 18). The increased survival observed 2 weeks after axotomy in animals treated with AAV.BAG1 may partly contribute to the better regeneration response. However, BAG1 transfected RGCs were not only increased in number, but also extended longer axons past the crush site. Moreover, our in vitro data shows that BAG1 overexpression enhances neurite outgrowth and modifies growth cone morphology by increasing growth cone area and the number of lamellipodia (Figures 19 and 20). Together, this suggests that BAG1 is not only neuroprotective, but also plays an important role in promoting regeneration by triggering cellular remodelling involved in neurite outgrowth.

\subsection{Subcellular translocation of Raf-1 and ROCK2 by BAG1}

BAG1 has been shown to bind to and activate the kinase Raf-1 (Wang et al., 1996; Song et al., 2001). In turn, Raf-1 was reported to colocalise and associate with ROCK2 and the lack of Raf- 1 resulted in the recruitment of ROCK2 to the cell membrane and ROCK2 hyperactivity (Ehrenreiter et al., 2005). Accordingly, our in vitro results show by immunocytochemistry and subcellular fractionation and in two different cell types that BAG1 overexpression induces Raf-1 translocation from a membrane-bound localisation to the cytoplasm (Figures 21, 22 and 24). Furthermore, ROCK2 translocates from the membrane to the perinuclear region. 
Following activation, Raf-1 has been reported to undergo intracellular redistribution, to the perinuclear space in murine fibroblastic NIH3T3 (Rapp et al., 1988) and rat hepatic Ito cells (Lissoos et al., 1993), into the nucleus in gerbil hippocampal pyramidal cells (Olah et al., 1991) and human megakaryoblastic leukaemia cells (Brennscheidt et al., 1994). Our subcellular fractionation results are in line with a previous report showing a mostly cytoplasmic and partly perinuclear localisation of Raf-1 in brain lysates (Morice et al., 1999). Thus, overexpression of BAG1 triggers Raf-1 translocation, recruiting this protein to a functionally different cellular compartment.

Our data also show that ROCK2 translocates from a membrane-bound localisation to the perinuclear and nuclear region upon BAG1 overexpression in primary RGCs and CSM cells. ROCK2 is reported to be essentially cytosolic and to translocate to the membrane upon Rhoactivation (Leung et al., 1996; Matsui et al., 1996). In fibroblasts, ROCK2 is found mostly at the cell membrane and in the perinuclear space (Yoneda et al., 2005), while recent studies also observed ROCK in the nucleus associated with the cyclin-dependent phosphatase Cdc25A or p300 acetyltransferase, thereby modulating histone activity (Bhowmick et al., 2003; Tanaka et al., 2006). These results suggest that, while active at the membrane under physiological conditions, ROCK2 translocation to the cytosol and perinuclear space might result in enzymatic inhibition and possible yet unknown functions in regulating gene transcription. Moreover, ROCK2 partially colocalises with cofilin, consistent with its well described role in actin stabilisation via activation of cofilin (Müller et al., 2005).

\subsection{Regulation of ROCK activity by BAG1 via Raf-1}

\subsubsection{Involvement of Raf-1 and the mitogen-activated protein kinase (MAPK) pathway}

In our in vitro paradigms, BAG1 overexpression activates Raf-1 and its downstream effector ERK, which is in agreement with previous studies (Wang et al., 1996; Song et al., 2001; Kermer et al., 2002; Liman et al., 2005). Ras-mediated activation of Raf-1 is specifically involved in axon lengthening of sensory neurons (Markus et al., 2002), which is triggered by microtubule polymerisation and phosphorylated (active) ERK2 translocation to the nucleus. ERK regulation of gene transcription plays a major role in developmental axon growth (Pearson et al., 2000). In rat pheochromocytoma-12 cells, activation of the MEK/ERK cascade, together with activation of the c-Jun N-terminal kinase (JNK) family, triggers NGFinduced neuronal differentiation, which is characterised by induction of neurofilaments involved in extension and stabilisation of neuronal processes (Lindenbaum et al., 1987; Clark 
and Lee, 1991), and by neurite extension (Heasley et al., 1996; Iwasaki et al., 1996). Accordingly, Raf-1 kinase inhibition reduced phospho-ERK expression in primary RGCs (Figure 28) and impaired axon outgrowth. Our results thus suggest that BAG1-induced regeneration is mediated by $\operatorname{Raf}-1$.

Interestingly, the $\mathrm{JNK}$ isoforms (comprising $\mathrm{JNK} 1,2$, and 3) play a differential role in survival and regeneration of neurons (Waetzig et al., 2006). Inhibition of JNK results in decreased neurite outgrowth (Eom et al., 2005). RNA interference studies showed that all three JNK isoforms are involved in the regenerative response of dopaminergic neurons in vitro, with a most dominant role of JNK3 (Planchamp et al., 2007). Consistently, levels of phosphorylated (active) JNK were moderately increased in CSM cells upon BAG1 overexpression (Figure 23), suggesting a possible involvement of the JNK pathway in the BAG1-mediated regenerative response. From the data generated in this study, we cannot determine whether JNK acts downstream of BAG1 or on a parallel level. The lack of sufficient isoform-specific and commercially available antibodies did not permit to further investigate a possible involvement of JNK in the BAG1-mediated regenerative response. However, no direct interaction between both pathways has been reported to date.

\subsubsection{Regulation of ROCK activity by Raf-1}

BAG1 overexpression significantly reduces ROCK activity in vitro, although ROCK2 expression levels remain stable within the cell. This effect does not involve RhoA, an upstream activator of ROCK. RhoA expression in differentiated cells, indeed, did not vary upon BAG1 overexpression (Figure 23). We can though hypothesize that the moderate increase in RhoA levels observed in non-differentiated BAG1 overexpressing cells may account for the small degree of process formation in this undifferentiated state.

On the other hand, we could demonstrate that BAG1-mediated reduction in ROCK activity is dependent on Raf-1 kinase. Application of Raf-1 kinase inhibitor increases ROCK activity in wild-type and BAG1 overexpressing cells (Figure 27), suggesting that Raf-1 is responsible for the BAG1-mediated reduction in ROCK activity in CSM cells. The N-terminal regulatory domain of Raf-1, which contains a pleckstrin domain homolog to ROCK2 C-terminal autoregulatory region, was shown to inhibit the kinase domain of ROCK2 (Baccarini, 2005). In contrast, the Raf-1 kinase domain is not involved in ROCK interaction (Ehrenreiter et al., 2005). This suggests that the sterical impairment in the Raf-1/ROCK interaction through 
inhibitor binding results in a reduction of ROCK inhibition, even though we cannot rule out that the observed effect on ROCK activity is at least partially mediated by inhibition of the kinase activity of Raf-1.

Furthermore, we could show that Raf-1 and ROCK2 partially colocalise (Figure 21), which is consistent with previous reports (Ehrenreiter et al., 2005). In addition, Raf-1 inhibition reverses the effects of BAG1 on neurite outgrowth and translocation of Raf-1 and ROCK2, which both remained membrane-bound (Figure 28). These results confirm that ROCK2 translocation and decreased activity are mediated by Raf-1. Raf-1 thus may also alter ROCK function by recruiting it to functionally different cellular compartments.

\subsection{Model of BAG1-mediated regenerative effects}

In summary, overexpression of BAG1 in vivo resulted in decreased lesion-induced apoptosis and increased regeneration. Based on our findings and the current literature, we describe a novel pathway through which BAG1 can regulate ROCK activity and propose a model for BAG1-mediated regeneration in vivo and neurite outgrowth in vitro (Figure 29).

Based on our findings, we postulate that BAG1 activates Raf-1, which can then lead to the activation of the MAPK pathway. Activation of ERK may play a role in enhancement of the intrinsic capacity of neurons to regenerate by stimulating neurite outgrowth, but might not be alone sufficient to overcome inhibitory signals impeding neurite outgrowth. On the other hand, active Raf-1 physically interacts with and inhibits ROCK activity. Under control or physiological conditions, Raf-1 levels do not significantly restrain ROCK activity, leading to limitations in outgrowth and regenerative potential. In contrast, overexpression of BAG1 stimulates Raf-1 activation and subsequent ROCK inhibition. This decreased enzymatic activity, in addition to the translocation of ROCK2 from the membrane to cytosolic and perinuclear spaces, may result in modulation of actin cytoskeleton and growth cones. It thus prevents growth cone collapse by blocking of inhibitory signalling. 


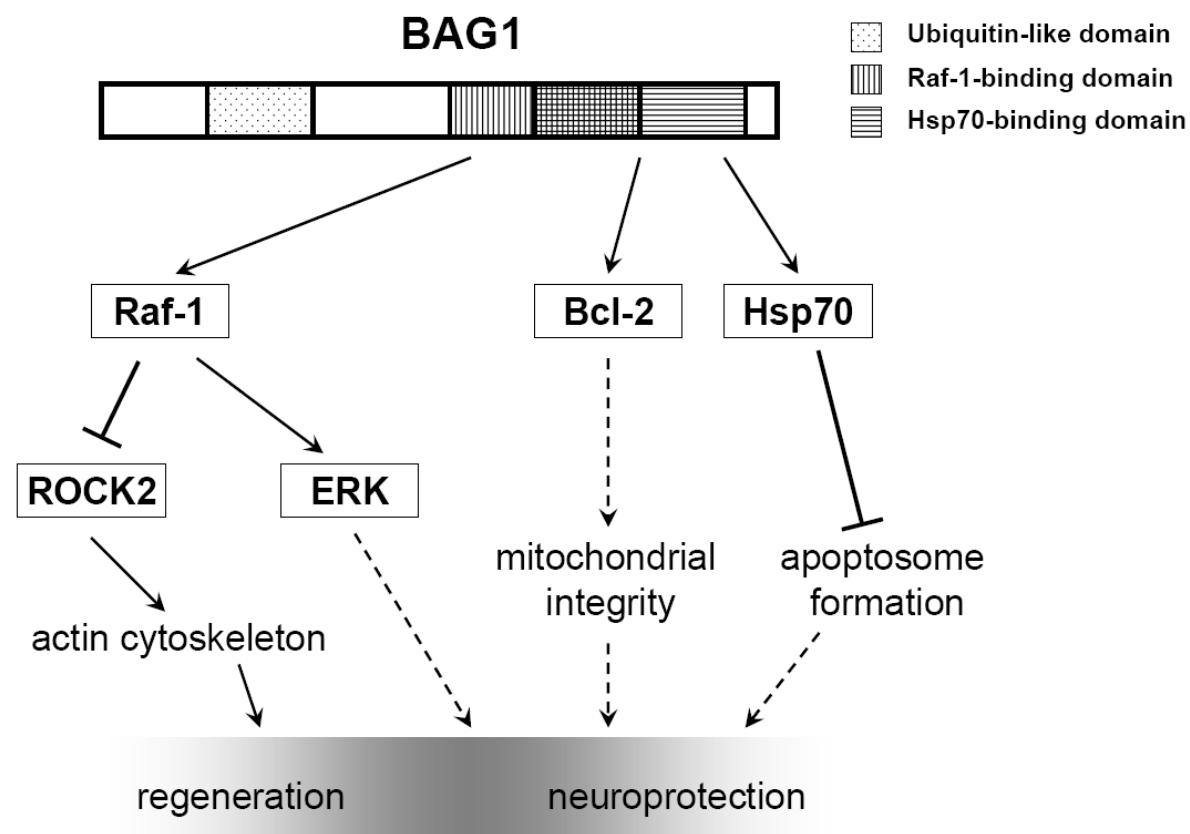

Figure 29: $\quad$ Model of BAG1-mediated regeneration and antiapoptosis based on the reported findings and the current literature. BAG1 activates Raf-1, which leads to the activation of the MAPK pathway and inhibition of ROCK activity. Activation of ERK may play a role in enhancement of the intrinsic capability of neurons to regenerate by stimulating neurite outgrowth. In contrast, a decrease in ROCK enzymatic activity, in addition to the translocation of ROCK2 from a membrane-bound to cytosolic and perinuclear space, may result in modulation of actin cytoskeleton and growth cones. Through its Hsp70-binding domain, BAG1 activates Bcl-2 and Hsp70, thus enhancing survival. Full lines, direct interaction; dashed lines, indirect interaction; arrow, stimulation; bar end, inhibition.

The mechanisms involved in BAG1-mediated neuroprotection are already well understood. They are based on the ability of BAG1 to coordinate the activity of both the chaperone and proteasome systems through its cochaperone activity, thereby facilitating or controlling protein refolding and turnover (Townsend et al., 2004; Liman et al., 2005). On one hand, BAG1 regulates the Hsp70/Hsc70 chaperone activity by binding to its ATPase domain (Takayama et al., 1997). Moreover, it supports the binding of the ligase complex to the proteasome through its ubiquitin-like domain and triggers the release of ubiquitinylated substrates from Hsc70/Hsp70 for their transfer to the proteasome (Alberti et al., 2002). BAG1 also plays a role in controlling the assembly and activation of the apoptosome (Townsend et al., 2005). Interaction of Hsp70 with Apaf-1 prevents the formation of the apoptosome and further activation of the intrinsic apoptosis pathway (Beere et al., 2000; Saleh et al., 2000). Additionally, BAG1 has been shown to stimulate the anti-apoptotic effects of Bcl-2, thereby preserving mitochondrial integrity (Schulz et al., 1997; Eversole-Cire et al., 2000; Takayama and Reed, 2001). 


\subsection{Neuroprotection and regeneration: achievements and perspectives}

\subsubsection{Achievements}

Restoration of function in the CNS upon injury or degenerative demise crucially depends on the simultaneous targeting of apoptosis and growth inhibition. Experimental treatment strategies aiming at the inhibition of apoptosis unfortunately result only in temporary cell survival and do not approach axonal regrowth. At the same time, strategies focusing solely on regeneration and axonal integrity require the presence of functionally active, living cells, which are reduced in number following lesion. For example, AAV-2-mediated expression of the anti-apoptotic molecule $\mathrm{Bcl}-\mathrm{X}_{\mathrm{L}}$ resulted in $\mathrm{RGC}$ survival rates which were comparable to our results at day 14 post-axotomy (Malik et al., 2005). However, Bcl- $\mathrm{X}_{\mathrm{L}}$ expression alone failed to enhance axonal growth in retinal explants (Dietz et al., 2006). Most neurotrophic factors improve cell survival but are not sufficient to promote regeneration (Mey and Thanos, 1993; Mansour-Robaey et al., 1994; Peinado-Ramon et al., 1996; Di Polo et al., 1998). AAVmediated expression of CNTF has been shown to potently increase the number of surviving RGCs and to promote regeneration (Leaver et al., 2006b). However, growth factors such as CNTF mainly act via inhibition of lesion-induced apoptosis and increase intrinsic regenerative capacity of adult neurons (Park et al., 2004), while growth inhibitory cascades are only moderately modulated (Lingor et al., submitted).

To achieve functional restoration, the most promising approach to consider is therefore to combine different strategies aiming at (1) preventing lesion-induced apoptosis, (2) increasing the intrinsic regenerative potential of CNS neurons and (3) suppressing inhibitory growth signalling from the environment. Recent studies already showed that synergistic effects can be achieved by combining neurotrophic factors, ROCK inhibitors or anti-apoptotic molecules. For example, application of CNTF accompanied by pharmacological inhibition of ROCK acts in a synergistic manner to increase regeneration of RGCs in semi-permissive and nonpermissive environments (Lingor et al., submitted). Similarly, AAV-mediated expression of $\mathrm{CNTF}$ in $\mathrm{Bcl}-2^{+/+}$transgenic mice shows synergistic effects on RGC survival and regeneration (Leaver et al., 2006a).

Here, we investigated the effects of BAG1, which we have shown to modulate functional restoration at three different levels by simultaneously increasing cell survival, enhancing the intrinsic regeneration potential and reducing inhibitory signalling via ROCK. In vivo overexpression of BAG1 resulted in a significant increase of RGC survival at 14 days post- 
axotomy and induced a marked regenerative response under non-permissive conditions. Our data on regeneration are in line with previous results obtained with pharmacological inhibition of ROCK2 by $\mathrm{Y}-27632$, which was shown to increase RGC regeneration in vivo (Lingor et al., 2007). Nevertheless, regeneration in these models remains moderate (axon regrowth in the order of $1 \mathrm{~mm}$ ), and is likely to remain insufficient to induce functional restoration in the human CNS. One of the possible improvements for future studies may be achieved by application of higher virus titres which would allow the transduction of larger and clinically relevant areas. For example, in the context of $\alpha$-1-antitrypsin ${ }^{2}$ (AAT) deficiency, an ongoing phase I study based on AAV-2-mediated overexpression of AAT includes doses up to $7 \times 10^{13}$ vector genomes (corresponding to approximately $2.3 \times 10^{12}$ i.u.) per patient (Flotte et al., 2004).

On the other hand, alternative signalling cascades independent from ROCK might also be involved in the inhibition of neurite outgrowth, which have not been addressed in this study. For example, ephrins and semaphorins signal through activation of the glycogen synthase kinase-3 $\beta$ (GSK-3 $\beta$ ) in a manner independent of the ROCK pathway (Chadborn et al., 2006; Ito et al., 2006). GSK-3 $\beta$ itself regulates microfilament dynamics and is involved in axon remodelling (Lucas et al., 1998; Sayas et al., 1999). Therefore, inhibition of one pathway alone might not be sufficient for clinically relevant regeneration.

\subsubsection{Perspectives}

A better regenerative response might be achieved by further combining a treatment based on BAG1 overexpression with other molecules targeting these different aspects. For example, combination with a vector expressing the anti-apoptotic $\mathrm{Bcl}-\mathrm{X}_{\mathrm{L}}$ could possibly increase the pool of surviving cells, thus resulting in an even stronger regenerative response. Additional supply of neurotrophic factors such as CNTF could further increase the intrinsic regenerative potential of neurons. Inactivation of different inhibitory pathways may in addition result in a better axonal outgrowth. Reduction of inhibitory signalling such as Rho or GSK-3 $\beta$ pathways could be achieved by downregulation of specific targets (e.g. RhoA) by AAV vectors expressing short hairpin RNA (Michel et al., 2005). In the future, a combination of multiple targets to regulate different pathways simultaneously may prove more promising than the modulation of one pathway alone.

\footnotetext{
${ }^{2} \alpha$-1-antitrypsin deficiency is due to a missense mutation leading to defects in the secretion of the protease. This leads to a loss of interstitial elastin and chronic airway inflammation, and results in a chronic obstructive pulmonary disease in adulthood.
} 
In this study, we used AAV-mediated gene transfer which allowed for specific targeting of RGCs in our in vivo models. With respect to potential therapeutic approaches, this technique represents the advantage of a local and sustained delivery of the protein of interest in a specific cell population. The use of the human synapsin promoter ensures physiological levels of protein expression (Kügler et al., 2003a). Modulation of the expression by different serotypes allows the targeting of specific cell types such as dopaminergic neurons (Shevtsova et al., 2005). Moreover, simultaneous application of different viruses encoding different proteins has been demonstrated in vivo (Michel et al., 2005). This may allow combining treatments with two or three different targets.

In the present work, we have used two widely established in vivo models to evaluate the effects of BAG1 overexpression, the optic nerve axotomy and crush models. Both techniques are straightforward to implement and allowed us to test in a reproducible manner the effects of BAG1 in vivo. It would now be interesting to translate BAG1 regenerative properties into a more therapeutic context. An approach which comes closer to human pathology is the spinal cord injury model. In this model, animal are submitted to a small dorsolateral laminectomy and consequent hemisection of the spinal cord, where regeneration of dorsal root ganglion neurons can be evaluated. Primary sensory neurons with cell bodies in the dorsal root ganglion are constituted of two axonal branches; a peripheral axonal branch regenerating upon injury and a central branch which enters the CNS and fails to show a pronounced regenerative response, similar to the axons of the optic nerve (Ramon y Cajal, 1928; Schnell and Schwab, 1990; Xu et al., 1995). These differential regenerative abilities would allow for comparative analysis of regeneration following lesion and the administration of different treatments, such as viral vectors. 


\section{Summary and concluding remarks}

In the present work, we investigated BAG1 as a therapeutic molecule in the lesioned visual system in vivo. More precisely, we tested the effects of BAG1 in two in vivo models of apoptosis and regeneration, the optic nerve axotomy and optic nerve crush. BAG1 was overexpressed in RGCs under the control of a neuron-specific promoter using AAV vectors. AAV-mediated overexpression of BAG1 significantly increased RGC survival compared to PBS and AAV.EGFP-treated controls. These results were confirmed in BAG1 ${ }^{+/-}$transgenic mice. BAG1 overexpression also significantly increased in vivo regeneration and in vitro neurite outgrowth of RGCs. Following AAV.BAG1 application, we observed a significant increase in the number and length of regenerating axons after optic nerve crush. In primary RGCs, BAG1 overexpression resulted in an approximately three-fold increase in neurite length and growth cone surface.

Further in vitro studies in two different cell types, the primary RGCs and the rat neuronal CSM cell line stably transfected with BAG1, allowed us to gain insights into the molecular mechanisms of BAG1-mediated regeneration. BAG1 induced translocation of Raf-1 and ROCK2 from the membrane to the cytoskeleton and the perinuclear regions, respectively. Most importantly, ROCK activity was significantly reduced in a Raf-1-dependent manner upon BAG1 overexpression.

In summary, we show that BAG1 acts on three different levels, by inhibition of lesioninduced apoptosis, increase of the intrinsic regeneration potential and interaction with the inhibitory ROCK signalling cascade. Thus, BAG1 is a promising candidate for the development of future therapeutic strategies targeting CNS regeneration in vivo. 


\section{References}

Alavi A, Hood JD, Frausto R, Stupack DG, Cheresh DA (2003) Role of Raf in vascular protection from distinct apoptotic stimuli. Science 301:94-96.

Alberti S, Demand J, Esser C, Emmerich N, Schild H, Hohfeld J (2002) Ubiquitylation of BAG1 suggests a novel regulatory mechanism during the sorting of chaperone substrates to the proteasome. J Biol Chem 277:45920-45927.

Amano M, Chihara K, Kimura K, Fukata Y, Nakamura N, Matsuura Y, Kaibuchi K (1997) Formation of actin stress fibers and focal adhesions enhanced by Rho-kinase. Science 275:1308-1311.

Amano M, Ito M, Kimura K, Fukata Y, Chihara K, Nakano T, Matsuura Y, Kaibuchi K (1996) Phosphorylation and activation of myosin by Rho-associated kinase (Rhokinase). Journal of Biological Chemistry 271:20246-20249.

Amano M, Kaneko T, Maeda A, Nakayama M, Ito M, Yamauchi T, Goto H, Fukata Y, Oshiro N, Shinohara A, Iwamatsu A, Kaibuchi K (2003) Identification of Tau and MAP2 as novel substrates of Rho-kinase and myosin phosphatase. Journal of Neurochemistry 87:780-790.

Antonsson B, Montessuit S, Sanchez B, Martinou JC (2001) Bax is present as a high molecular weight oligomer/complex in the mitochondrial membrane of apoptotic cells. J Biol Chem 276:11615-11623.

Antonsson B, Montessuit S, Lauper S, Eskes R, Martinou JC (2000) Bax oligomerization is required for channel-forming activity in liposomes and to trigger cytochrome c release from mitochondria. Biochem J 345 Pt 2:271-278.

Arimura N, Inagaki N, Chihara $\mathrm{K}$, Menager C, Nakamura N, Amano M, Iwamatsu A, Goshima Y, Kaibuchi K (2000) Phosphorylation of collapsin response mediator protein-2 by Rho-kinase - Evidence for two separate signaling pathways for growth cone collapse. Journal of Biological Chemistry 275:23973-23980.

Baccarini M (2005) Second nature: biological functions of the Raf-1 "kinase". FEBS Lett 579:3271-3277.

Bähr M (2000) Live or let die - retinal ganglion cell death and survival during development and in the lesioned adult CNS. Trends Neurosci 23:483-490.

Bähr M, Lingor P (2006) Brain repair: Experimental treatment strategies, neuroprotective and repair strategies in the lesioned adult CNS. Adv Exp Med Biol 557:148-163.

Bandtlow CE, Schmidt MF, Hassinger TD, Schwab ME, Kater SB (1993) Role of intracellular calcium in NI-35-evoked collapse of neuronal growth cones. Science 259:80-83.

Bardelli A, Longati P, Albero D, Goruppi S, Schneider C, Ponzetto C, Comoglio PM (1996) HGF receptor associates with the anti-apoptotic protein BAG1 and prevents cell death. Embo J 15:6205-6212.

Barres BA, Silverstein BE, Corey DP, Chun LL (1988) Immunological, morphological, and electrophysiological variation among retinal ganglion cells purified by panning. Neuron 1:791-803.

Barton WA, Liu BP, Tzvetkova D, Jeffrey PD, Fournier AE, Sah D, Cate R, Strittmatter SM, Nikolov DB (2003) Structure and axon outgrowth inhibitor binding of the Nogo-66 receptor and related proteins. Embo J 22:3291-3302.

Bartsch U, Bandtlow CE, Schnell L, Bartsch S, Spillmann AA, Rubin BP, Hillenbrand R, Montag D, Schwab ME, Schachner M (1995) Lack of evidence that myelin-associated glycoprotein is a major inhibitor of axonal regeneration in the CNS. Neuron 15:13751381. 
Beere HM, Wolf BB, Cain K, Mosser DD, Mahboubi A, Kuwana T, Tailor P, Morimoto RI, Cohen GM, Green DR (2000) Heat-shock protein 70 inhibits apoptosis by preventing recruitment of procaspase-9 to the Apaf-1 apoptosome. Nat Cell Biol 2:469-475.

Benson MD, Romero MI, Lush ME, Lu QR, Henkemeyer M, Parada LF (2005) Ephrin-B3 is a myelin-based inhibitor of neurite outgrowth. Proc Natl Acad Sci U S A 102:1069410699.

Berkelaar M, Clarke DB, Wang YC, Bray GM, Aguayo AJ (1994) Axotomy results in delayed death and apoptosis of retinal ganglion cells in adult rats. J Neurosci 14:43684374.

Bertrand J, Winton MJ, Rodriguez-Hernandez N, Campenot RB, McKerracher L (2005) Application of Rho antagonist to neuronal cell bodies promotes neurite growth in compartmented cultures and regeneration of retinal ganglion cell axons in the optic nerve of adult rats. J Neurosci 25:1113-1121.

Bhowmick NA, Ghiassi M, Aakre M, Brown K, Singh V, Moses HL (2003) TGF-betainduced RhoA and p160ROCK activation is involved in the inhibition of Cdc25A with resultant cell-cycle arrest. Proc Natl Acad Sci U S A 100:15548-15553.

Bomze HM, Bulsara KR, Iskandar BJ, Caroni P, Skene JH (2001) Spinal axon regeneration evoked by replacing two growth cone proteins in adult neurons. Nat Neurosci 4:38-43.

Brennscheidt U, Riedel D, Kolch W, Bonifer R, Brach MA, Ahlers A, Mertelsmann RH, Herrmann F (1994) Raf-1 is a necessary component of the mitogenic response of the human megakaryoblastic leukemia cell line MO7 to human stem cell factor, granulocyte-macrophage colony-stimulating factor, interleukin 3, and interleukin 9. Cell Growth Differ 5:367-372.

Bretscher A (1999) Regulation of cortical structure by the ezrin-radixin-moesin protein family. Current Opinion in Cell Biology 11:109-116.

Broude E, McAtee M, Kelley MS, Bregman BS (1997) c-Jun expression in adult rat dorsal root ganglion neurons: differential response after central or peripheral axotomy. Exp Neurol 148:367-377.

Buller RM, Janik JE, Sebring ED, Rose JA (1981) Herpes simplex virus types 1 and 2 completely help adenovirus-associated virus replication. J Virol 40:241-247.

Burnette WN (1981) "Western blotting": electrophoretic transfer of proteins from sodium dodecyl sulfate--polyacrylamide gels to unmodified nitrocellulose and radiographic detection with antibody and radioiodinated protein A. Anal Biochem 112:195-203.

Cearley CN, Wolfe JH (2006) Transduction characteristics of adeno-associated virus vectors expressing cap serotypes 7, 8, 9, and Rh10 in the mouse brain. Mol Ther 13:528-537.

Cellerino A, Galli-Resta L, Colombaioni L (2000a) The dynamics of neuronal death: a timelapse study in the retina. J Neurosci 20:RC92.

Cellerino A, Bähr M, Isenmann S (2000b) Apoptosis in the developing visual system. Cell Tissue Res 301:53-69.

Ceulemans H, Bollen M (2004) Functional diversity of protein phosphatase-1, a cellular economizer and reset button. Physiol Rev 84:1-39.

Chadborn NH, Ahmed AI, Holt MR, Prinjha R, Dunn GA, Jones GE, Eickholt BJ (2006) PTEN couples Sema3A signalling to growth cone collapse. J Cell Sci 119:951-957.

Chaudhary P, Ahmed F, Quebada P, Sharma SC (1999) Caspase inhibitors block the retinal ganglion cell death following optic nerve transection. Brain Res Mol Brain Res 67:3645.

Chen XQ, Tan I, Ng CH, Hall C, Lim L, Leung T (2002) Characterization of RhoA-binding kinase ROK alpha implication of the pleckstrin homology domain in ROK alpha function using region-specific antibodies. Journal of Biological Chemistry 277:1268012688 . 
Cheng EH, Wei MC, Weiler S, Flavell RA, Mak TW, Lindsten T, Korsmeyer SJ (2001) Bcl2, Bcl- $\mathrm{X}_{\mathrm{L}}$ sequester $\mathrm{BH} 3$ domain-only molecules preventing BAX- and BAKmediated mitochondrial apoptosis. Mol Cell 8:705-711.

Chin PC, Liu L, Morrison BE, Siddiq A, Ratan RR, Bottiglieri T, D'Mello SR (2004) The cRaf inhibitor GW5074 provides neuroprotection in vitro and in an animal model of neurodegeneration through a MEK-ERK and Akt-independent mechanism. J Neurochem 90:595-608.

Choi DW (1988) Glutamate neurotoxicity and diseases of the nervous system. Neuron 1:623634.

Chong H, Guan KL (2003) Regulation of Raf through phosphorylation and N terminus-C terminus interaction. J Biol Chem 278:36269-36276.

Clark EA, Lee VM (1991) The differential role of protein kinase C isozymes in the rapid induction of neurofilament phosphorylation by nerve growth factor and phorbol esters in PC12 cells. J Neurochem 57:802-810.

Clevenger CV, Thickman K, Ngo W, Chang WP, Takayama S, Reed JC (1997) Role of BAG1 in the survival and proliferation of the cytokine-dependent lymphocyte lines, $\mathrm{Ba} / \mathrm{F} 3$ and $\mathrm{Nb} 2$. Mol Endocrinol 11:608-618.

Cohen-Cory S, Fraser SE (1995) Effects of brain-derived neurotrophic factor on optic axon branching and remodelling in vivo. Nature 378:192-196.

Coleman MP, Perry VH (2002) Axon pathology in neurological disease: a neglected therapeutic target. Trends Neurosci 25:532-537.

Crocoll A, Blum M, Cato AC (2000) Isoform-specific expression of BAG1 in mouse development. Mech Dev 91:355-359.

Danial NN, Korsmeyer SJ (2004) Cell death: critical control points. Cell 116:205-219.

David S, Aguayo AJ (1981) Axonal elongation into peripheral nervous system "bridges" after central nervous system injury in adult rats. Science 214:931-933.

de Hoop MJ, Meyn, L., Dotti, C.G. (1998) In: Culturing hippocampal neurons and astrocytes from fetal rodent brain. San Diego: Academic Press.

de Rijk MC, Launer LJ, Berger K, Breteler MM, Dartigues JF, Baldereschi M, Fratiglioni L, Lobo A, Martinez-Lage J, Trenkwalder C, Hofman A (2000) Prevalence of Parkinson's disease in Europe: A collaborative study of population-based cohorts. Neurologic Diseases in the Elderly Research Group. Neurology 54:S21-23.

Dergham P, Ellezam B, Essagian C, Avedissian H, Lubell WD, McKerracher L (2002) Rho signaling pathway targeted to promote spinal cord repair. J Neurosci 22:6570-6577.

Dhillon AS, Kolch W (2002) Untying the regulation of the Raf-1 kinase. Arch Biochem Biophys 404:3-9.

Dhillon AS, von Kriegsheim A, Grindlay J, Kolch W (2007) Phosphatase and feedback regulation of Raf-1 signaling. Cell Cycle 6:3-7.

Di Polo A, Aigner LJ, Dunn RJ, Bray GM, Aguayo AJ (1998) Prolonged delivery of brainderived neurotrophic factor by adenovirus-infected Muller cells temporarily rescues injured retinal ganglion cells. Proc Natl Acad Sci U S A 95:3978-3983.

Diamond J, Holmes M, Coughlin M (1992) Endogenous NGF and nerve impulses regulate the collateral sprouting of sensory axons in the skin of the adult rat. J Neurosci 12:14541466.

Dietz GP, Dietz B, Bähr M (2006) Bcl-X $\mathrm{X}_{\mathrm{L}}$ increases axonal numbers but not axonal elongation from rat retinal explants. Brain Res Bull 70:117-123.

Dragunow M, Xu R, Walton M, Woodgate A, Lawlor P, MacGibbon GA, Young D, Gibbons H, Lipski J, Muravlev A, Pearson A, During M (2000) c-Jun promotes neurite outgrowth and survival in PC12 cells. Brain Res Mol Brain Res 83:20-33. 
Du C, Fang M, Li Y, Li L, Wang X (2000) Smac, a mitochondrial protein that promotes cytochrome c-dependent caspase activation by eliminating IAP inhibition. Cell 102:33-42.

Ehrenreiter K, Piazzolla D, Velamoor V, Sobczak I, Small JV, Takeda J, Leung T, Baccarini M (2005) Raf-1 regulates Rho signaling and cell migration. J Cell Biol 168:955-964.

Ermak G, Davies KJ (2002) Calcium and oxidative stress: from cell signaling to cell death. Mol Immunol 38:713-721.

Eversole-Cire P, Concepcion FA, Simon MI, Takayama S, Reed JC, Chen J (2000) Synergistic effect of Bcl-2 and BAG1 on the prevention of photoreceptor cell death. Invest Ophthalmol Vis Sci 41:1953-1961.

Fawcett JW (1997) Astrocytic and neuronal factors affecting axon regeneration in the damaged central nervous system. Cell Tissue Res 290:371-377.

Fernandes KJ, Fan DP, Tsui BJ, Cassar SL, Tetzlaff W (1999) Influence of the axotomy to cell body distance in rat rubrospinal and spinal motoneurons: differential regulation of GAP43, tubulins, and neurofilament-M. J Comp Neurol 414:495-510.

Filbin MT (2003) Myelin-associated inhibitors of axonal regeneration in the adult mammalian CNS. Nat Rev Neurosci 4:703-713.

Fitch MT, Silver J (1997) Glial cell extracellular matrix: boundaries for axon growth in development and regeneration. Cell Tissue Res 290:379-384.

Flotte TR, Afione SA, Zeitlin PL (1994) Adeno-associated virus vector gene expression occurs in nondividing cells in the absence of vector DNA integration. Am J Respir Cell Mol Biol 11:517-521.

Flotte TR, Brantly ML, Spencer LT, Byrne BJ, Spencer CT, Baker DJ, Humphries M (2004) Phase I trial of intramuscular injection of a recombinant adeno-associated virus alpha 1-antitrypsin (rAAV2-CB-hAAT) gene vector to AAT-deficient adults. Hum Gene Ther 15:93-128.

Foo RS, Mani K, Kitsis RN (2005) Death begets failure in the heart. J Clin Invest 115:565571.

Fournier AE, GrandPre T, Strittmatter SM (2001) Identification of a receptor mediating Nogo-66 inhibition of axonal regeneration. Nature 409:341-346.

Fournier AE, Takizawa BT, Strittmatter SM (2003) Rho kinase inhibition enhances axonal regeneration in the injured CNS. J Neurosci 23:1416-1423.

Fujisawa K, Fujita A, Ishizaki T, Saito Y, Narumiya S (1996) Identification of the Rhobinding domain of p160(ROCK), a Rho-associated coiled-coil containing protein kinase. Journal of Biological Chemistry 271:23022-23028.

Garcia-Valenzuela E, Gorczyca W, Darzynkiewicz Z, Sharma SC (1994) Apoptosis in adult retinal ganglion cells after axotomy. J Neurobiol 25:431-438.

Goldshmit Y, Galea MP, Wise G, Bartlett PF, Turnley AM (2004) Axonal regeneration and lack of astrocytic gliosis in EphA4-deficient mice. J Neurosci 24:10064-10073.

Götz R, Wiese S, Takayama S, Camarero GC, Rossoll W, Schweizer U, Troppmair J, Jablonka S, Holtmann B, Reed JC, Rapp UR, Sendtner M (2005) Bag1 is essential for differentiation and survival of hematopoietic and neuronal cells. Nat Neurosci 8:11691178.

GrandPre T, Li S, Strittmatter SM (2002) Nogo-66 receptor antagonist peptide promotes axonal regeneration. Nature 417:547-551.

GrandPre T, Nakamura F, Vartanian T, Strittmatter SM (2000) Identification of the Nogo inhibitor of axon regeneration as a Reticulon protein. Nature 403:439-444.

Grimm D, Kern A, Rittner K, Kleinschmidt JA (1998) Novel tools for production and purification of recombinant adenoassociated virus vectors. Hum Gene Ther 9:27452760 . 
Gross A, Yin XM, Wang K, Wei MC, Jockel J, Milliman C, Erdjument-Bromage H, Tempst $\mathrm{P}$, Korsmeyer SJ (1999) Caspase cleaved BID targets mitochondria and is required for cytochrome $\mathrm{c}$ release, while $\mathrm{Bcl}-\mathrm{X}_{\mathrm{L}}$ prevents this release but not tumor necrosis factorR1/Fas death. J Biol Chem 274:1156-1163.

Hakem R, Hakem A, Duncan GS, Henderson JT, Woo M, Soengas MS, Elia A, de la Pompa JL, Kagi D, Khoo W, Potter J, Yoshida R, Kaufman SA, Lowe SW, Penninger JM, Mak TW (1998) Differential requirement for caspase 9 in apoptotic pathways in vivo. Cell 94:339-352.

Hanahan D (1983) Studies on transformation of Escherichia coli with plasmids. J Mol Biol 166:557-580.

Haralson MA, Hassel JR (1995) In: Extracellular matrix: a practical approach. Ithaca, N.Y.: IRL Press.

He XL, Bazan JF, McDermott G, Park JB, Wang K, Tessier-Lavigne M, He Z, Garcia KC (2003) Structure of the Nogo receptor ectodomain: a recognition module implicated in myelin inhibition. Neuron 38:177-185.

Heasley LE, Storey B, Fanger GR, Butterfield L, Zamarripa J, Blumberg D, Maue RA (1996) GTPase-deficient $\mathrm{G}$ alpha 16 and $\mathrm{G}$ alpha $\mathrm{q}$ induce PC12 cell differentiation and persistent activation of cJun NH2-terminal kinases. Mol Cell Biol 16:648-656.

Hirose M, Ishizaki T, Watanabe N, Uehata M, Kranenburg O, Moolenaar WH, Matsumura F, Maekawa M, Bito H, Narumiya S (1998) Molecular dissection of the Rho-associated protein kinase (p160ROCK)-regulated neurite remodeling in neuroblastoma N1E-115 cells. Journal of Cell Biology 141:1625-1636.

Hohfeld J, Jentsch S (1997) GrpE-like regulation of the hsc70 chaperone by the anti-apoptotic protein BAG1. Embo J 16:6209-6216.

Hsieh SH, Ferraro GB, Fournier AE (2006) Myelin-associated inhibitors regulate cofilin phosphorylation and neuronal inhibition through LIM kinase and Slingshot phosphatase. J Neurosci 26:1006-1015.

Huber AB, Schwab ME (2000) Nogo-A, a potent inhibitor of neurite outgrowth and regeneration. Biol Chem 381:407-419.

Hull M, Bähr M (1994) Differential regulation of c-Jun expression in rat retinal ganglion cells after proximal and distal optic nerve transection. Neurosci Lett 178:39-42.

Iannotti C, Li H, Yan P, Lu X, Wirthlin L, Xu XM (2003) Glial cell line-derived neurotrophic factor-enriched bridging transplants promote propriospinal axonal regeneration and enhance myelination after spinal cord injury. Exp Neurol 183:379-393.

Inoue H, Nojima H, Okayama H (1990) High efficiency transformation of Escherichia coli with plasmids. Gene 96:23-28.

Isenmann S, Bähr M (1997) Expression of c-Jun protein in degenerating retinal ganglion cells after optic nerve lesion in the rat. Exp Neurol 147:28-36.

Isenmann S, Wahl C, Krajewski S, Reed JC, Bähr M (1997) Up-regulation of Bax protein in degenerating retinal ganglion cells precedes apoptotic cell death after optic nerve lesion in the rat. Eur J Neurosci 9:1763-1772.

Ishizaki T, Maekawa M, Fujisawa K, Okawa K, Iwamatsu A, Fujita A, Watanabe N, Saito Y, Kakizuka A, Morii N, Narumiya S (1996) The small GTP-binding protein Rho binds to and activates a $160 \mathrm{kDa} \mathrm{Ser} / \mathrm{Thr}$ protein kinase homologous to myotonic dystrophy kinase. Embo Journal 15:1885-1893.

Ito Y, Oinuma I, Katoh H, Kaibuchi K, Negishi M (2006) Sema4D/plexin-B1 activates GSK3beta through R-Ras GAP activity, inducing growth cone collapse. EMBO Rep 7:704709.

Iwasaki S, Hattori A, Sato M, Tsujimoto M, Kohno M (1996) Characterization of the bone morphogenetic protein-2 as a neurotrophic factor. Induction of neuronal differentiation 
of PC12 cells in the absence of mitogen-activated protein kinase activation. J Biol Chem 271:17360-17365.

Jalink K, van Corven EJ, Hengeveld T, Morii N, Narumiya S, Moolenaar WH (1994) Inhibition of lysophosphatidate- and thrombin-induced neurite retraction and neuronal cell rounding by ADP ribosylation of the small GTP-binding protein Rho. J Cell Biol 126:801-810.

Jansen HW, Ruckert B, Lurz R, Bister K (1983) 2 Unrelated Cell-Derived Sequences in the Genome of Avian Leukemia and Carcinoma Inducing Retrovirus Mh2. Embo Journal 2:1969-1975.

Janssens V, Goris J (2001) Protein phosphatase 2A: a highly regulated family of serine/threonine phosphatases implicated in cell growth and signalling. Biochem $\mathbf{J}$ 353:417-439.

Jin S, Zhuo Y, Guo W, Field J (2005) p21-activated Kinase 1 (Pak1)-dependent phosphorylation of Raf-1 regulates its mitochondrial localization, phosphorylation of BAD, and Bcl-2 association. J Biol Chem 280:24698-24705.

Juliano RL, Reddig P, Alahari S, Edin M, Howe A, Aplin A (2004) Integrin regulation of cell signalling and motility. Biochem Soc Trans 32:443-446.

Kawabata S, Usukura J, Morone N, Ito M, Iwamatsu A, Kaibuchi K, Amano M (2004) Interaction of Rho-kinase with myosin II at stress fibres. Genes to Cells 9:653-660.

Kawano Y, Fukata Y, Oshiro N, Amano M, Nakamura T, Ito M, Matsumura F, Inagaki M, Kaibuchi K (1999) Phosphorylation of myosin-binding subunit (MBS) of myosin phosphatase by Rho-kinase in vivo. J Cell Biol 147:1023-1038.

Kermer P, Klocker N, Labes M, Bähr M (1998) Inhibition of CPP32-like proteases rescues axotomized retinal ganglion cells from secondary cell death in vivo. J Neurosci 18:4656-4662.

Kermer P, Klocker N, Weishaupt JH, Bähr M (2001) Transection of the optic nerve in rats: studying neuronal death and survival in vivo. Brain Res Brain Res Protoc 7:255-260.

Kermer P, Klocker N, Labes M, Thomsen S, Srinivasan A, Bähr M (1999) Activation of caspase-3 in axotomized rat retinal ganglion cells in vivo. FEBS Lett 453:361-364.

Kermer P, Ankerhold R, Klocker N, Krajewski S, Reed JC, Bähr M (2000) Caspase-9: involvement in secondary death of axotomized rat retinal ganglion cells in vivo. Brain Res Mol Brain Res 85:144-150.

Kermer P, Krajewska M, Zapata JM, Takayama S, Mai J, Krajewski S, Reed JC (2002) Bag1 is a regulator and marker of neuronal differentiation. Cell Death Differ 9:405-413.

Kermer P, Digicaylioglu MH, Kaul M, Zapata JM, Krajewska M, Stenner-Liewen F, Takayama S, Krajewski S, Lipton SA, Reed JC (2003) BAG1 over-expression in brain protects against stroke. Brain Pathol 13:495-506.

Kerr JF, Wyllie AH, Currie AR (1972) Apoptosis: a basic biological phenomenon with wideranging implications in tissue kinetics. Br J Cancer 26:239-257.

Kim JE, Li S, GrandPre T, Qiu D, Strittmatter SM (2003) Axon regeneration in young adult mice lacking Nogo-A/B. Neuron 38:187-199.

Kimura K, Ito M, Amano M, Chihara K, Fukata Y, Nakafuku M, Yamamori B, Feng JH, Nakano T, Okawa K, Iwamatsu A, Kaibuchi K (1996) Regulation of myosin phosphatase by Rho and Rho-Associated kinase (Rho-kinase). Science 273:245-248.

Klocker N, Braunling F, Isenmann S, Bähr M (1997) In vivo neurotrophic effects of GDNF on axotomized retinal ganglion cells. Neuroreport 8:3439-3442.

Knee DA, Froesch BA, Nuber U, Takayama S, Reed JC (2001) Structure-function analysis of Bag1 proteins. Effects on androgen receptor transcriptional activity. J Biol Chem 276:12718-12724.

Koeberle PD, Ball AK (1998) Effects of GDNF on retinal ganglion cell survival following axotomy. Vision Res 38:1505-1515. 
Krammer PH (1999) CD95(APO-1/Fas)-mediated apoptosis: live and let die. Adv Immunol 71:163-210.

Kügler S, Kilic E, Bähr M (2003a) Human synapsin 1 gene promoter confers highly neuronspecific long-term transgene expression from an adenoviral vector in the adult rat brain depending on the transduced area. Gene Ther 10:337-347.

Kügler S, Lingor P, Scholl U, Zolotukhin S, Bähr M (2003b) Differential transgene expression in brain cells in vivo and in vitro from AAV-2 vectors with small transcriptional control units. Virology 311:89-95.

Kügler S, Meyn L, Holzmuller H, Gerhardt E, Isenmann S, Schulz JB, Bähr M (2001) Neuron-specific expression of therapeutic proteins: evaluation of different cellular promoters in recombinant adenoviral vectors. Mol Cell Neurosci 17:78-96.

Kullmann M, Schneikert J, Moll J, Heck S, Zeiner M, Gehring U, Cato AC (1998) RAP46 is a negative regulator of glucocorticoid receptor action and hormone-induced apoptosis. J Biol Chem 273:14620-14625.

Kuo WL, Abe M, Rhee J, Eves EM, McCarthy SA, Yan M, Templeton DJ, McMahon M, Rosner MR (1996) Raf, but not MEK or ERK, is sufficient for differentiation of hippocampal neuronal cells. Mol Cell Biol 16:1458-1470.

Kureishi Y, Kobayashi S, Amano M, Kimura K, Kanaide H, Nakano T, Kaibuchi K, Ito M (1997) Rho-associated kinase directly induces smooth muscle contraction through myosin light chain phosphorylation. Journal of Biological Chemistry 272:1225712260.

Lackey K, Cory M, Davis R, Frye SV, Harris PA, Hunter RN, Jung DK, McDonald OB, McNutt RW, Peel MR, Rutkowske RD, Veal JM, Wood ER (2000) The discovery of potent cRafl kinase inhibitors. Bioorg Med Chem Lett 10:223-226.

Leaver SG, Cui Q, Bernard O, Harvey AR (2006a) Cooperative effects of Bcl-2 and AAVmediated expression of CNTF on retinal ganglion cell survival and axonal regeneration in adult transgenic mice. Eur J Neurosci 24:3323-3332.

Leaver SG, Cui Q, Plant GW, Arulpragasam A, Hisheh S, Verhaagen J, Harvey AR (2006b) AAV-mediated expression of CNTF promotes long-term survival and regeneration of adult rat retinal ganglion cells. Gene Ther 13:1328-1341.

Leevers SJ, Paterson HF, Marshall CJ (1994) Requirement for Ras in Raf activation is overcome by targeting Raf to the plasma membrane. Nature 369:411-414.

Lehmann M, Fournier A, Selles-Navarro I, Dergham P, Sebok A, Leclerc N, Tigyi G, McKerracher L (1999) Inactivation of Rho signaling pathway promotes CNS axon regeneration. J Neurosci 19:7537-7547.

Leung T, Chen XQ, Manser E, Lim L (1996) The p160 RhoA-binding kinase ROK alpha is a member of a kinase family and is involved in the reorganization of the cytoskeleton. Molecular and Cellular Biology 16:5313-5327.

Li H, Zhu H, Xu CJ, Yuan J (1998) Cleavage of BID by caspase 8 mediates the mitochondrial damage in the Fas pathway of apoptosis. Cell 94:491-501.

Liman J, Ganesan S, Dohm CP, Krajewski S, Reed JC, Bähr M, Wouters FS, Kermer P (2005) Interaction of BAG1 and Hsp70 mediates neuroprotectivity and increases chaperone activity. Mol Cell Biol 25:3715-3725.

Lindenbaum MH, Carbonetto S, Mushynski WE (1987) Nerve growth factor enhances the synthesis, phosphorylation, and metabolic stability of neurofilament proteins in PC12 cells. J Biol Chem 262:605-610.

Lindwall C, Kanje M (2005a) The Janus role of c-Jun: cell death versus survival and regeneration of neonatal sympathetic and sensory neurons. Exp Neurol 196:184-194.

Lindwall C, Kanje M (2005b) The role of p-c-Jun in survival and outgrowth of developing sensory neurons. Neuroreport 16:1655-1659. 
Lingor P, Koeberle P, Kügler S, Bähr M (2005) Down-regulation of apoptosis mediators by RNAi inhibits axotomy-induced retinal ganglion cell death in vivo. Brain 128:550558.

Lingor P, Teusch N, Schwarz K, Müller R, Mack H, Bähr M, Müller BK (2007) Inhibition of Rho kinase (ROCK) increases neurite outgrowth on chondroitin sulphate proteoglycan in vitro and axonal regeneration in the adult optic nerve in vivo. J Neurochem 103:181-9.

Lissoos TW, Beno DWA, Davis BH (1993) 1,25-Dihydroxyvitamin-D(3) Activates Raf Kinase and Raf Perinuclear Translocation Via a Protein-Kinase C-Dependent Pathway. Journal of Biological Chemistry 268:25132-25138.

Liu R, Takayama S, Zheng Y, Froesch B, Chen GQ, Zhang X, Reed JC, Zhang XK (1998) Interaction of BAG1 with retinoic acid receptor and its inhibition of retinoic acidinduced apoptosis in cancer cells. J Biol Chem 273:16985-16992.

Lucas FR, Goold RG, Gordon-Weeks PR, Salinas PC (1998) Inhibition of GSK-3beta leading to the loss of phosphorylated MAP-1B is an early event in axonal remodelling induced by WNT-7a or lithium. J Cell Sci 111 ( Pt 10):1351-1361.

Luders J, Demand J, Schonfelder S, Frien M, Zimmermann R, Hohfeld J (1998) Cofactorinduced modulation of the functional specificity of the molecular chaperone Hsc70. Biol Chem 379:1217-1226.

Luo X, Budihardjo I, Zou H, Slaughter C, Wang X (1998) Bid, a Bcl-2 interacting protein, mediates cytochrome c release from mitochondria in response to activation of cell surface death receptors. Cell 94:481-490.

Maekawa M, Ishizaki T, Boku S, Watanabe N, Fujita A, Iwamatsu A, Obinata T, Ohashi K, Mizuno K, Narumiya S (1999) Signaling from rho to the actin cytoskeleton through protein kinases ROCK and LIM-kinase. Science 285:895-898.

Malik JM, Shevtsova Z, Bähr M, Kügler S (2005) Long-term in vivo inhibition of CNS neurodegeneration by Bcl- $X_{\mathrm{L}}$ gene transfer. Mol Ther 11:373-381.

Mansour-Robaey S, Clarke DB, Wang YC, Bray GM, Aguayo AJ (1994) Effects of ocular injury and administration of brain-derived neurotrophic factor on survival and regrowth of axotomized retinal ganglion cells. Proc Natl Acad Sci U S A 91:16321636.

Markus A, Zhong J, Snider WD (2002) Raf and Akt mediate distinct aspects of sensory axon growth. Neuron 35:65-76.

Martins LM, Iaccarino I, Tenev T, Gschmeissner S, Totty NF, Lemoine NR, Savopoulos J, Gray CW, Creasy CL, Dingwall C, Downward J (2002) The serine protease $\mathrm{Omi} / \mathrm{HtrA} 2$ regulates apoptosis by binding XIAP through a reaper-like motif. J Biol Chem 277:439-444.

Mason MR, Campbell G, Caroni P, Anderson PN, Lieberman AR (2000) Overexpression of GAP43 in thalamic projection neurons of transgenic mice does not enable them to regenerate axons through peripheral nerve grafts. Exp Neurol 165:143-152.

Matsui T, Amano M, Yamamoto T, Chihara K, Nakafuku M, Ito M, Nakano T, Okawa K, Iwamatsu A, Kaibuchi K (1996) Rho-associated kinase, a novel serine threonine kinase, as a putative target for the small GTP binding protein Rho. Embo Journal 15:2208-2216.

Matsuzawa S, Takayama S, Froesch BA, Zapata JM, Reed JC (1998) p53-inducible human homologue of Drosophila seven in absentia (Siah) inhibits cell growth: suppression by BAG1. Embo J 17:2736-2747.

Maxam AM, Gilbert W (1977) A new method for sequencing DNA. Proc Natl Acad Sci U S A 74:560-564. 
McKeon RJ, Schreiber RC, Rudge JS, Silver J (1991) Reduction of neurite outgrowth in a model of glial scarring following CNS injury is correlated with the expression of inhibitory molecules on reactive astrocytes. J Neurosci 11:3398-3411.

Meberg PJ, Bamburg JR (2000) Increase in neurite outgrowth mediated by overexpression of actin depolymerizing factor. Journal of Neuroscience 20:2459-2469.

Mey J, Thanos S (1993) Intravitreal injections of neurotrophic factors support the survival of axotomized retinal ganglion cells in adult rats in vivo. Brain Res 602:304-317.

Michel U, Malik I, Ebert S, Bähr M, Kügler S (2005) Long-term in vivo and in vitro AAV-2mediated RNA interference in rat retinal ganglion cells and cultured primary neurons. Biochem Biophys Res Commun 326:307-312.

Mills JC, Stone NL, Erhardt J, Pittman RN (1998) Apoptotic membrane blebbing is regulated by myosin light chain phosphorylation. J Cell Biol 140:627-636.

Monnier PP, Sierra A, Schwab JM, Henke-Fahle S, Mueller BK (2003) The Rho/ROCK pathway mediates neurite growth-inhibitory activity associated with the chondroitin sulfate proteoglycans of the CNS glial scar. Mol Cell Neurosci 22:319-330.

Morice C, Nothias F, Konig S, Vernier P, Baccarini M, Vincent JD, Barnier JV (1999) Raf-1 and B-Raf proteins have similar regional distributions but differential subcellular localization in adult rat brain. Eur J Neurosci 11:1995-2006.

Müller BK, Mack H, Teusch N (2005) Rho kinase, a promising drug target for neurological disorders. Nat Rev Drug Discov 4:387-398.

Nakagawa O, Fujisawa K, Ishizaki T, Saito Y, Nakao K, Narumiya S (1996) ROCK-I and ROCK-II, two isoforms of Rho-associated coiled-coil forming protein serine/threonine kinase in mice. Febs Letters 392:189-193.

Narumiya S, Ishizaki T, Watanabe N (1997) Rho effectors and reorganization of actin cytoskeleton. FEBS Lett 410:68-72.

Ng J, Luo L (2004) Rho GTPases regulate axon growth through convergent and divergent signaling pathways. Neuron 44:779-793.

Niederost B, Oertle T, Fritsche J, McKinney RA, Bandtlow CE (2002) Nogo-A and myelinassociated glycoprotein mediate neurite growth inhibition by antagonistic regulation of RhoA and Rac1. J Neurosci 22:10368-10376.

Niederost BP, Zimmermann DR, Schwab ME, Bandtlow CE (1999) Bovine CNS myelin contains neurite growth-inhibitory activity associated with chondroitin sulfate proteoglycans. J Neurosci 19:8979-8989.

Ohashi K, Nagata K, Maekawa M, Ishizaki T, Narumiya S, Mizuno K (2000) Rho-associated kinase ROCK activates LIM-kinase 1 by phosphorylation at threonine 508 within the activation loop. Journal of Biological Chemistry 275:3577-3582.

Olah Z, Komoly S, Nagashima N, Joo F, Rapp UR, Anderson WB (1991) Cerebral ischemia induces transient intracellular redistribution and intranuclear translocation of the raf proto-oncogene product in hippocampal pyramidal cells. Exp Brain Res 84:403-410.

Oppenheim RW (1996) The concept of uptake and retrograde transport of neurotrophic molecules during development: history and present status. Neurochem Res 21:769777.

Park JB, Yiu G, Kaneko S, Wang J, Chang J, He XL, Garcia KC, He Z (2005) A TNF receptor family member, TROY, is a coreceptor with Nogo receptor in mediating the inhibitory activity of myelin inhibitors. Neuron 45:345-351.

Park K, Luo JM, Hisheh S, Harvey AR, Cui Q (2004) Cellular mechanisms associated with spontaneous and ciliary neurotrophic factor-cAMP-induced survival and axonal regeneration of adult retinal ganglion cells. J Neurosci 24:10806-10815.

Park S, Rath O, Beach S, Xiang X, Kelly SM, Luo Z, Kolch W, Yeung KC (2006) Regulation of RKIP binding to the N-region of the Raf-1 kinase. FEBS Lett 580:6405-6412. 
Paves H, Saarma M (1997) Neurotrophins as in vitro growth cone guidance molecules for embryonic sensory neurons. Cell Tissue Res 290:285-297.

Pearson G, Bumeister R, Henry DO, Cobb MH, White MA (2000) Uncoupling Raf1 from MEK1/2 impairs only a subset of cellular responses to Raf activation. J Biol Chem 275:37303-37306.

Peinado-Ramon P, Salvador M, Villegas-Perez MP, Vidal-Sanz M (1996) Effects of axotomy and intraocular administration of NT-4, NT-3, and brain-derived neurotrophic factor on the survival of adult rat retinal ganglion cells. A quantitative in vivo study. Invest Ophthalmol Vis Sci 37:489-500.

Planchamp V, Bähr M, Lingor P (2007) Differential role of c-Jun-N-terminal kinase isoforms in regeneration and survival of primary dopaminergic neurons. Neuroforum February 2007 (1) vol. 13 T3-2B.

Potts RA, Dreher B, Bennett MR (1982) The loss of ganglion cells in the developing retina of the rat. Brain Res 255:481-486.

Prouty SM, Maroo A, Maucher C, Mischak H, Kolch W, Sedivy JM (1998) Studies of perinuclear and nuclear translocation of the Raf-1 protein in rodent fibroblasts. Biochimica Et Biophysica Acta-Molecular Cell Research 1402:6-16.

Raivich G, Bohatschek M, Da Costa C, Iwata O, Galiano M, Hristova M, Nateri AS, Makwana M, Riera-Sans L, Wolfer DP, Lipp HP, Aguzzi A, Wagner EF, Behrens A (2004) The AP-1 transcription factor c-Jun is required for efficient axonal regeneration. Neuron 43:57-67.

Ramon y Cajal S (1928) Degeneration and regeneration of the nervous system. Translated by R.M. May, edited by J. DeFelipe and E.G. Jones, 1991. New York: Oxford University Press.

Rapp UR, Heidecker G, Huleihel M, Cleveland JL, Choi WC, Pawson T, Ihle JN, Anderson WB (1988) Raf Family Serine Threonine Protein-Kinases in Mitogen Signal Transduction. Cold Spring Harbor Symposia on Quantitative Biology 53:173-184.

Robinson GA (1994) Immediate early gene expression in axotomized and regenerating retinal ganglion cells of the adult rat. Brain Res Mol Brain Res 24:43-54.

Roux P, Menguy I, Soubigou S, Chinn J, Ricard S, Williams S, Guitton JD, Tian T, Singh S, Grepin C (2004) Direct measurement of multiple mRNAs in nerve growth factorinduced PC12 cells using electrophoretic tags to monitor biomarkers of neuronal differentiation in 96-well format. Assay Drug Dev Technol 2:637-646.

Sabel BA, Aschoff A (1993) Functional recovery and morphological changes after injury to the optic nerve. Neuropsychobiology 28:62-65.

Saleh A, Srinivasula SM, Balkir L, Robbins PD, Alnemri ES (2000) Negative regulation of the Apaf-1 apoptosome by Hsp70. Nat Cell Biol 2:476-483.

Sayas CL, Moreno-Flores MT, Avila J, Wandosell F (1999) The neurite retraction induced by lysophosphatidic acid increases Alzheimer's disease-like Tau phosphorylation. J Biol Chem 274:37046-37052.

Schmeer C, Straten G, Kügler S, Gravel C, Bähr M, Isenmann S (2002) Dose-dependent rescue of axotomized rat retinal ganglion cells by adenovirus-mediated expression of glial cell-line derived neurotrophic factor in vivo. Eur J Neurosci 15:637-643.

Schnell L, Schwab ME (1990) Axonal regeneration in the rat spinal cord produced by an antibody against myelin-associated neurite growth inhibitors. Nature 343:269-272.

Schreyer DJ, Skene JH (1993) Injury-associated induction of GAP43 expression displays axon branch specificity in rat dorsal root ganglion neurons. J Neurobiol 24:959-970.

Schulz JB, Bremen D, Reed JC, Lommatzsch J, Takayama S, Wullner U, Loschmann PA, Klockgether T, Weller M (1997) Cooperative interception of neuronal apoptosis by BCL-2 and BAG1 expression: prevention of caspase activation and reduced production of reactive oxygen species. J Neurochem 69:2075-2086. 
Schwab ME (2004) Nogo and axon regeneration. Curr Opin Neurobiol 14:118-124.

Schwab ME, Thoenen H (1985) Dissociated neurons regenerate into sciatic but not optic nerve explants in culture irrespective of neurotrophic factors. J Neurosci 5:2415-2423.

Shao Z, Browning JL, Lee X, Scott ML, Shulga-Morskaya S, Allaire N, Thill G, Levesque M, Sah D, McCoy JM, Murray B, Jung V, Pepinsky RB, Mi S (2005) TAJ/TROY, an orphan TNF receptor family member, binds Nogo-66 receptor 1 and regulates axonal regeneration. Neuron 45:353-359.

Shevtsova Z, Malik JM, Michel U, Bähr M, Kügler S (2005) Promoters and serotypes: targeting of adeno-associated virus vectors for gene transfer in the rat central nervous system in vitro and in vivo. Exp Physiol 90:53-59.

Shi J, Wei L (2007) Rho kinase in the regulation of cell death and survival. Arch Immunol Ther Exp (Warsz) 55:61-75.

Silver J, Miller JH (2004) Regeneration beyond the glial scar. Nat Rev Neurosci 5:146-156.

Skene JH, Virag I (1989) Posttranslational membrane attachment and dynamic fatty acylation of a neuronal growth cone protein, GAP43. J Cell Biol 108:613-624.

Sondermann H, Scheufler C, Schneider C, Hohfeld J, Hartl FU, Moarefi I (2001) Structure of a Bag/Hsc70 complex: convergent functional evolution of Hsp70 nucleotide exchange factors. Science 291:1553-1557.

Song J, Takeda M, Morimoto RI (2001) Bag1-Hsp70 mediates a physiological stress signalling pathway that regulates Raf-1/ERK and cell growth. Nat Cell Biol 3:276282.

Srivastava A, Lusby EW, Berns KI (1983) Nucleotide sequence and organization of the adeno-associated virus 2 genome. J Virol 45:555-564.

Stieger K, Le Meur G, Lasne F, Weber M, Deschamps JY, Nivard D, Mendes-Madeira A, Provost N, Martin L, Moullier P, Rolling F (2006) Long-term doxycycline-regulated transgene expression in the retina of nonhuman primates following subretinal injection of recombinant AAV vectors. Mol Ther 13:967-975.

Stokoe D, Macdonald SG, Cadwallader K, Symons M, Hancock JF (1994) Activation of Raf as a result of recruitment to the plasma membrane. Science 264:1463-1467.

Straten G, Schmeer C, Kretz A, Gerhardt E, Kügler S, Schulz JB, Gravel C, Bähr M, Isenmann S (2002) Potential synergistic protection of retinal ganglion cells from axotomy-induced apoptosis by adenoviral administration of glial cell line-derived neurotrophic factor and X-chromosome-linked inhibitor of apoptosis. Neurobiol Dis 11:123-133.

Sumi T, Matsumoto K, Nakamura T (2001) Specific activation of LIM kinase 2 via phosphorylation of threonine 505 by ROCK, a rho-dependent protein kinase. Journal of Biological Chemistry 276:670-676.

Takayama S, Reed JC (2001) Molecular chaperone targeting and regulation by BAG family proteins. Nat Cell Biol 3:E237-241.

Takayama S, Xie Z, Reed JC (1999) An evolutionarily conserved family of Hsp70/Hsc70 molecular chaperone regulators. J Biol Chem 274:781-786.

Takayama S, Sato T, Krajewski S, Kochel K, Irie S, Millan JA, Reed JC (1995) Cloning and functional analysis of BAG1: a novel Bcl-2-binding protein with anti-cell death activity. Cell 80:279-284.

Takayama S, Bimston DN, Matsuzawa S, Freeman BC, Aime-Sempe C, Xie Z, Morimoto RI, Reed JC (1997) BAG1 modulates the chaperone activity of Hsp70/Hsc70. Embo J 16:4887-4896.

Tanaka T, Nishimura D, Wu RC, Amano M, Iso T, Kedes L, Nishida H, Kaibuchi K, Hamamori Y (2006) Nuclear Rho kinase, ROCK2, targets p300 acetyltransferase. Journal of Biological Chemistry 281:15320-15329. 
Tenenbaum L, Chtarto A, Lehtonen E, Velu T, Brotchi J, Levivier M (2004) Recombinant AAV-mediated gene delivery to the central nervous system. J Gene Med 6 Suppl $1:$ S212-222.

Tom VJ, Steinmetz MP, Miller JH, Doller CM, Silver J (2004) Studies on the development and behavior of the dystrophic growth cone, the hallmark of regeneration failure, in an in vitro model of the glial scar and after spinal cord injury. J Neurosci 24:6531-6539.

Towbin H, Staehelin T, Gordon J (1979) Electrophoretic transfer of proteins from polyacrylamide gels to nitrocellulose sheets: procedure and some applications. Proc Natl Acad Sci U S A 76:4350-4354.

Townsend PA, Stephanou A, Packham G, Latchman DS (2005) BAG1: a multi-functional pro-survival molecule. Int J Biochem Cell Biol 37:251-259.

Townsend PA, Cutress RI, Sharp A, Brimmell M, Packham G (2003) BAG1: a multifunctional regulator of cell growth and survival. Biochim Biophys Acta 1603:8398.

Townsend PA, Cutress RI, Carroll CJ, Lawrence KM, Scarabelli TM, Packham G, Stephanou A, Latchman DS (2004) BAG1 proteins protect cardiac myocytes from simulated ischemia/reperfusion-induced apoptosis via an alternate mechanism of cell survival independent of the proteasome. J Biol Chem 279:20723-20728.

Tran NH, Frost JA (2003) Phosphorylation of Raf-1 by p21-activated kinase 1 and Src regulates Raf-1 autoinhibition. J Biol Chem 278:11221-11226.

Tsukita S, Yonemura S, Tsukita S (1997) ERM proteins: Head-to-tail regulation of actinplasma membrane interaction. Trends in Biochemical Sciences 22:53-58.

Tucker KL, Meyer M, Barde YA (2001) Neurotrophins are required for nerve growth during development. Nat Neurosci 4:29-37.

Van Hooff CO, Holthuis JC, Oestreicher AB, Boonstra J, De Graan PN, Gispen WH (1989) Nerve growth factor-induced changes in the intracellular localization of the protein kinase C substrate B-50 in pheochromocytoma PC12 cells. J Cell Biol 108:1115-1125.

Vaux DL, Korsmeyer SJ (1999) Cell death in development. Cell 96:245-254.

Verhagen AM, Ekert PG, Pakusch M, Silke J, Connolly LM, Reid GE, Moritz RL, Simpson RJ, Vaux DL (2000) Identification of DIABLO, a mammalian protein that promotes apoptosis by binding to and antagonizing IAP proteins. Cell 102:43-53.

Villegas-Perez MP, Vidal-Sanz M, Rasminsky M, Bray GM, Aguayo AJ (1993) Rapid and protracted phases of retinal ganglion cell loss follow axotomy in the optic nerve of adult rats. J Neurobiol 24:23-36.

Waetzig V, Zhao Y, Herdegen T (2006) The bright side of JNKs-Multitalented mediators in neuronal sprouting, brain development and nerve fiber regeneration. Prog Neurobiol 80:84-97.

Wang HG, Takayama S, Rapp UR, Reed JC (1996) Bcl-2 interacting protein, BAG1, binds to and activates the kinase Raf-1. Proc Natl Acad Sci U S A 93:7063-7068.

Watanabe M, Dykes-Hoberg M, Culotta VC, Price DL, Wong PC, Rothstein JD (2001) Histological evidence of protein aggregation in mutant SOD1 transgenic mice and in amyotrophic lateral sclerosis neural tissues. Neurobiol Dis 8:933-941.

Weise J, Isenmann S, Klocker N, Kügler S, Hirsch S, Gravel C, Bähr M (2000) Adenovirusmediated expression of ciliary neurotrophic factor (CNTF) rescues axotomized rat retinal ganglion cells but does not support axonal regeneration in vivo. Neurobiol Dis 7:212-223.

Wong ST, Henley JR, Kanning KC, Huang KH, Bothwell M, Poo MM (2002) A p75(NTR) and Nogo receptor complex mediates repulsive signaling by myelin-associated glycoprotein. Nat Neurosci 5:1302-1308. 
Xiang X, Zang M, Waelde CA, Wen R, Luo Z (2002) Phosphorylation of 338SSYY341 regulates specific interaction between Raf-1 and MEK1. J Biol Chem 277:4499645003.

Xu XM, Guenard V, Kleitman N, Aebischer P, Bunge MB (1995) A combination of BDNF and NT-3 promotes supraspinal axonal regeneration into Schwann cell grafts in adult rat thoracic spinal cord. Exp Neurol 134:261-272.

Yang N, Higuchi O, Ohashi K, Nagata K, Wada A, Kangawa K, Nishida E, Mizuno K (1998) Cofilin phosphorylation by LIM-kinase 1 and its role in Rac-mediated actin reorganization. Nature 393:809-812.

Yiu G, He Z (2003) Signaling mechanisms of the myelin inhibitors of axon regeneration. Curr Opin Neurobiol 13:545-551.

Yiu G, He Z (2006) Glial inhibition of CNS axon regeneration. Nat Rev Neurosci 7:617-627.

Yoneda A, Multhaupt HAB, Couchman JR (2005) The Rho kinases I and II regulate different aspects of myosin II activity. Journal of Cell Biology 170:443-453.

Zeiner M, Gehring U (1995) A protein that interacts with members of the nuclear hormone receptor family: identification and cDNA cloning. Proc Natl Acad Sci USA 92:1146511469.

Zeiner M, Gebauer M, Gehring U (1997) Mammalian protein RAP46: an interaction partner and modulator of $70 \mathrm{kDa}$ heat shock proteins. Embo J 16:5483-5490.

Zheng B, Ho C, Li S, Keirstead H, Steward O, Tessier-Lavigne M (2003) Lack of enhanced spinal regeneration in Nogo-deficient mice. Neuron 38:213-224.

Zheng B, Atwal J, Ho C, Case L, He XL, Garcia KC, Steward O, Tessier-Lavigne M (2005) Genetic deletion of the Nogo receptor does not reduce neurite inhibition in vitro or promote corticospinal tract regeneration in vivo. Proc Natl Acad Sci U S A 102:12051210.

Zhong LT, Sarafian T, Kane DJ, Charles AC, Mah SP, Edwards RH, Bredesen DE (1993) Bcl-2 inhibits death of central neural cells induced by multiple agents. Proc Natl Acad Sci U S A 90:4533-4537.

Zolotukhin S, Byrne BJ, Mason E, Zolotukhin I, Potter M, Chesnut K, Summerford C, Samulski RJ, Muzyczka N (1999) Recombinant adeno-associated virus purification using novel methods improves infectious titer and yield. Gene Ther 6:973-985.

Zou H, Li Y, Liu X, Wang X (1999) An APAF-1.cytochrome c multimeric complex is a functional apoptosome that activates procaspase-9. J Biol Chem 274:11549-11556. 


\section{Annexes}

\section{List of abbreviations}

$\underset{\varnothing}{1 \mathrm{x}}$

AAV.BAG1

AAV.EGFP

AAV-2

AD

ADF-cofilin

APS

ATP

BAG1

BCA

BDNF

bGH

BMP

bp

BSA

ca.

cap

cDNA

CHIP

$\mathrm{CMF}$

CNS

CNTF

CR

CRD

CREB

CSPG

DAPI

DEPC

DISC

DMEM

DMSO

DNA

DPBS

EBSS

ECM

EDTA

EGFP

EGTA

ELISA

ERK

FCS
Approximately

Diameter

One time

Adeno-associated virus expressing Flag-BAG1 and an EGFP reporter gene under the control of the hSyn1 promoter

Adeno-associated virus expressing an EGFP reporter gene under the control of the hSyn 1 promoter

Adeno-associated virus serotype 2

Alzheimer's disease

Actin depolymerising factor-cofilin

Ammonium peroxyde

Adenosine triphosphate

Bcl-2 associated athanogene-1

Bicinchoninic acid

Brain-derived neurotrophic factor

Bovine growth hormone

Bromophenol blue sodium salt

Base pair

Bovine serum albumin

Circa

Genes encoding AAV capside proteins

Complementary deoxyribonucleic acid

Carboxy terminus of $\mathrm{Hsc} 70$ interacting protein

$\mathrm{Ca}^{2+}-\mathrm{Mg}^{2+}$-Free medium

Central nervous system

Ciliary neurotrophic factor

Conserved domain

Cysteine-rich domain

cAMP replication element

Chondroitin sulphate proteoglycans

4', 6-diamidino-2-phenylindole

Diethyl pyrocarbonate

Death-inducing signalling complex

Dulbecco's modified Eagle's medium

Dimethyl sulfoxide

Deoxynucleic acid

Dulbecco's Phosphate Buffered Saline

Earle's Buffered Salt Solution

Extracellular matrix

Ethylenediamine-tetraacetic acid

Enhanced green fluorescence protein

Ethylenglycol-bis-(b-aminoethylether) N, N, N', N'-tetraacetic acid

Enzyme-linked immunosorbent assay

Extracellular signal-regulated kinase

Foetal calf serum 
$g$

GAP43

Gapdh

GDNF

$\mathrm{H}_{2} \mathrm{O}_{\text {dist. }}$

HBSS

$\mathrm{HC}$

HEPES

HRP

hSyn1

i.u

i.o.

Int

ITR

JNK

$\mathrm{Kb}$

$\mathrm{kDa}$

LB

LIMK

$\mathrm{M}$

MAG

MAPK

MEK

MBS

MLC

$\mathrm{NF \kappa B}$

NGF

NGS

NSE

OD

OMgp

pAAV-6P1-TB

PAGE

PBS

PCR

PD

PFA

pFlag-BAG1

$\mathrm{PH}$

PSN

PNS

P-ORN

RBD

rep

RGC

ROCK

ROCK2

rpm

RT
Relative centrifuge force

Growth-associated protein 43

Glyceraldehyde-3-phosphate dehydrogenase

Glial cell line-derived neurotrophic factor

Distilled and UV-purified RNAse-free water

Hanks Balanced Salt Solution

Hippocampal cell

4-(2-hydroethyl)-1-piperazineethanesulfonic acid

Horse radish peroxydase

Human synapsin promoter 1

Infective units

Intravitreal

Intron

Inverted terminal repeats

c-Jun N-terminal kinase

Kilobase pair

Kilodaltons

Luria broth medium

LIM kinase

Molar

Myelin-associated glycoprotein

Mitogen-activated protein kinase

Mitogen-activated and extracellular-regulated kinase

Myosin-biding subunit of myosin phosphatase

Myosin light chain

Nuclear factor-kappa B

Neuronal growth factor

New-born goat serum

Neuron-specific enolase promoter

Optical density

Oligodendrocyte myelin glycoprotein

Plasmid containing the AAV expression cassette constituted of

$\mathrm{Bcl}-\mathrm{X}_{\mathrm{L}} \mathrm{cDNA}$ and an EGFP reporter gene under the control of two independent hSyn 1 promoters

Polyacrylamide gel

Phosphate buffer solution

Polymerase chain reaction

Parkinson's disease

Paraformaldehyde

Plasmid containing the mouse Flag-tagged BAG1 cDNA

Pleckstrin homology

Penicillin-streptomycin-neomycin

Peripheral nervous system

Poly-D-ornithine

Rho- or RasGTP-loaded-binding domain

Genes responsible of the replication of AAV genome

Retinal ganglion cell

Rho-associated kinase (refers to both isoforms)

Rho-associated kinase 2

Rotation per minute

Room temperature 
SDS

SDS-PAGE

SV40

TB

TBE

TBS

TBS-T

TEMED

TNF $\alpha$

ULD

UV

WPRE

XIAP
Sodium dodecyl sulphate

Sodium dodecyl sulphate-polyacrylamide gel

Simian virus 40 large $T$ antigen

Synthetic posttranscriptional blocker

Tris-Borate-EDTA

Tris-buffered saline

Tris-buffered saline-Tween

$\mathrm{N}, \mathrm{N}, \mathrm{N}$ ', N'-tetrametylethylene diamine

Tumor necrosis factor alpha

Ubiquitin-like domain

Ultraviolet

Woodchuck hepatitis virus posttranslational control element $\mathrm{X}$-linked inhibitor of apoptosis protein 


\section{List of Publications}

Planchamp V, Bermel C, Tönges L, Kügler S, Kermer P, Reed JC, Bähr M, and Lingor P (submitted) BAG1 promotes axonal outgrowth and regeneration in vivo by activation of Raf-1 and reduction of ROCK activity.

Lingor P, Tönges L, Piper N, Bermel C, Planchamp V, and Bähr M (submitted) CNTF and ROCK inhibition increase survival and regeneration response of retinal ganglion cells in vitro and in vivo.

Meuer K, Lingor P, Görricke B, Planchamp V, Bähr M, Weishaupt JH (2007) CDK5 promotes mitochondrial fission during neuronal apoptosis. Cell Death Differ 14:651-61.

Pietrancosta N, Moumen A, Dono R, Lingor P, Planchamp V, Lamballe F, Bähr M, Kraus JL, and Maina F (2006) Mechanism of action of the imino-tetrahydro-benzothiazole JLK 1179, a novel inhibitor of p53-induced neuronal death in vivo. J Med Chem 49:3645-52.

\section{Poster abstracts}

Planchamp V, Bähr M, and Lingor P (2007) Differential role of c-Jun-N-terminal kinase isoforms in regeneration and survival of primary dopaminergic neurons. Neuroforum February 2007 (1) vol. 13 T3-2B. Presented at the $31^{\text {st }}$ Göttingen Neurobiology Conference, March 29-April $1^{\text {st }} 2007$, Göttingen, Germany.

Planchamp V, Tönges L, Kügler S, Kermer P, Bähr M, and Lingor P (2006) Role of Bcl-2 associated athanogene-1 in antiapoptosis and regeneration of retinal ganglion cells. FENS Abstracts vol. 3 A202.209. Presented at the $5^{\text {th }}$ Forum of European Neuroscience, July 8-12, 2006, Vienna, Austria. 


\section{Acknowledgements}

I would like to express my gratitude to:

Prof. Mathias Bähr, who welcomed me in his laboratory and made this work possible.

Dr. Paul Lingor, for his availability and his excellent supervision, as well as for creating a nice working atmosphere in the group.

Prof. Rüdiger Hardeland and Prof. Gerhard Braus, who agreed to take over the Referat and Correferat. I especially thank Prof. Hardeland for valuable feedback on the project.

Dr. Pawel Kermer, for making the experiments possible by providing me with the pFlagBAG1 plasmid. Additionally, he kindly shared CSM cells and several antibodies.

Dr. Sebastian Kügler, who produced the viral vectors and was always there for constructive discussions and sharing his views on science.

Prof. Uwe Michel, for helpful discussions and sharing tips on cloning techniques.

Many thanks to Elisabeth Barski, Ulricke Schöll, Alexandra Marten and Beate Veigh, for their availability, technical help and keeping the lab running so well.

My colleagues and friends from the "S2-Labor": Christina Bermel, Lars Tönges, Jan Koch, Johanna Knöferle, Ligia Ferreira, Manuel Garrido and Zinayida Shevtsova, for the very nice working atmosphere and the good time spent outside the lab... A special thank you to Christina, who established the primary retinal ganglion cell culture in our group, to Lars, who double-counted the RGCs and to Zina, who took time to critically read some parts of the manuscript.

My friends from the NSR network, for their scientific input and the wonderful moments we shared all around Europe during these years of $\mathrm{PhD} \ldots$

My dear friends Carina Alcala, Anjana Nityanandam and Ivana Gadjanski, who were always there to share good and sad moments, regardless of the distance or the time of day...

My parents, brother and grand-parents, for their continuous love, support and encouragement, despite the distance parting us.

Last but not least, Henry, for his patience and his support in so many things that I cannot list them all here... 


\section{Curriculum vitae}

\section{Personal details:}

Name: Anne-Véronique Planchamp

Citizenship: Swiss

Date of birth: $\quad 20^{\text {th }}$ May 1978

Place of birth: $\quad$ Vevey, Switzerland

Marital status: $\quad$ single

\section{Education:}

2004-2007 PhD education

Laboratory of Prof. Bähr, Dept. of Neurology, University of Göttingen, Germany

2002-2003 Licence ès sciences (= BSc in Physics)

Faculty of Sciences, University of Lausanne, Switzerland

1996-2002 MSc in Biology

Faculty of Sciences, University of Lausanne, Switzerland

1993-1996 High-school diploma Latin-ancient Greek

High school of Burier, La Tour-de-Peilz, Switzerland 